\title{
D-Branes, T-Duality, and Index Theory
}

\author{
Kentaro Hori \\ Department of Physics, University of California at Berkeley \\ 366 Le Conte Hall, Berkeley, CA 94720-7300, U.S.A. \\ and \\ Theoretical Physics Group, Mail Stop 50A-5101 \\ Ernest Orlando Lawrence Berkeley National Laboratory \\ Berkeley, CA 94720, U.S.A.
}

\begin{abstract}
We show that the transformation of D-branes under T-duality on four-torus is represented by Nahm transform of instantons. The argument for this allows us to generalize Nahm transform to the case of orthogonal and symplectic gauge groups as well as to instantons on $\mathbf{Z}_{2}$ orbifold of four-torus. In addition, we identify the isomorphism of $\mathrm{K}$-theory groups that realizes the transformation of D-brane charges under T-duality on torus of arbitrary dimensions. By the isomorphism we are led to identify the correct K-theory group that classifies D-brane charges in Type II orientifold.
\end{abstract}




\section{Introduction}

A D $p$-brane wrapped on a circle is mapped under T-duality to a $\mathrm{D}(p-$ 1)-brane where the $U(1)$ Wilson line of the $\mathrm{D} p$-brane corresponds to the position of the $\mathrm{D}(p-1)$-brane in the dual circle. This was known since the discovery of D-branes $[1,2]$ and this is actually how D-branes are discovered. However, the T-duality transformation rule is less obvious for more general configuration of D-branes in a more general string background.

In superstring theory, D-branes are sources of Ramond-Ramond (RR) potentials [3]. Transformation of RR fields under T-duality was studied in $[4,5]$. RR fields of the theory on $T^{n} \times M$ and those of the $\mathrm{T}$-dual theory on $\widehat{T}^{n} \times M$ are related as

$$
\widehat{\mathrm{e}^{-B} F}=\int_{T^{n}} \operatorname{ch}(\mathcal{P}) \mathrm{e}^{-B} F
$$

Here, $B$ is the Neveu-Schwarz B-field and $F=\sum_{p} F_{p+2}$ is the sum of gauge invariant $\mathrm{RR}$ field strengths where the sum is over $p=0,2,4, \ldots$ for Type IIA and $p=-1,1,3, \ldots$ for Type IIB. ${ }^{1}$ Also, $\operatorname{ch}(\mathcal{P})=\exp \left(\sum_{i=1}^{n} \mathrm{~d} \widehat{t_{i}} \wedge \mathrm{d} t^{i}\right)$ in which $t^{i}$ and $\widehat{t}_{i}$ are coordinates of $T^{n}$ and $\widehat{T}^{n}$ dual to each other. As the name suggests, $\operatorname{ch}(\mathcal{P})$ represents the Chern character of a complex line bundle over $\widehat{T}^{n} \times T^{n}$ - the so called Poincaré bundle $\mathcal{P}$ - which has a $U(1)$ connection with curvature $-2 \pi i \sum_{i} \mathrm{~d} \widehat{t_{i}} \wedge \mathrm{d} t^{i}$.

The simplicity of the transformation rule (1.1) is intriguing and begs for an explanation which is independent of the supergravity or worldsheet computation. It is also interesting to ask whether similar rule exists for more general cases, such as the case with orbifold or orientifold projection. We note here that (1.1) looks like a formula which appears in the family index theory [6-8]; in particular, for a family of Dirac operators on $T^{n}$ parametrized by $\widehat{T}^{n}$ which is carried by a bundle over $T^{n} \times \widehat{T}^{n}$ determined by $\mathcal{P}$. Since D-branes are RR sources, this suggests that there is a similar index-theoretic transformation rule for D-branes, which might lead to a simplest explanation for (1.1) and to its generalization. The purpose of this paper is to find such a transformation rule for D-brane configurations.

\footnotetext{
${ }^{1}$ We choose the following field definitions. The gauge transformation for the $\mathrm{R}-\mathrm{R}$ potential $A=\sum A_{p+1}$ is given by $\delta A=\mathrm{e}^{B} \mathrm{~d} \alpha$ for $\alpha=\sum \alpha_{p}$, and therefore the gauge invariant field strength is $F=\mathrm{e}^{B} \mathrm{~d}\left(\mathrm{e}^{-B} A\right)=\mathrm{d} A-H \wedge A$ subject to the Bianchi identity $\mathrm{d} F=H \wedge F$.
} 
Actually, it has been suspected that an index-theoretic transformation realizes T-duality on four-torus $T^{4}$ (and also $T^{n}$ with $n=1,2,3$ ) in the case where there are $\mathrm{D} p$-branes at points on $T^{4}$ within wrapped $\mathrm{D}(p+4)$ branes. In such a case, a D-brane configuration can be represented as an instanton configuration on $T^{4}$, and it was suggested in [9] that $\mathrm{T}$-duality may possibly be realized as a transform of representing instanton configurations called Nahm transform [11-13] (or Mukai's Fourier transform [10]). Nahm transform uses a family of Dirac operators on $T^{4}$ parametrized by $\widehat{T}^{4}$ which is carried by the Poincaré bundle $\mathcal{P}$, and the transformation of the topological numbers of the instantons is given by an index formula which looks like (1.1). In particular, the rank and the instanton number are interchanged. In terms of D-brane charges, this corresponds to the interchange of $(p+4)$-brane and $p$-brane charges which is exactly what T-duality does. However, there was no other argument showing why the Nahm transform can be identified with the T-duality on four-torus. In this paper, we provide a simple argument which shows that one can indeed identify the Nahm transform and T-duality. The argument is so simple that it can be easily generalized to the case with orbifold/orientifold projection, finding a generalization of the $\mathrm{Nahm}$ transform to, say, orthogonal/symplectic gauge groups. . Since $D^{n, 0} \times D^{0, n} \cong$ $D^{n, n}$

Based on recent analysis of brane-anti-brane systems (for example in [14]), it was argued by Witten [15] that, as has been proposed in [16], Dbrane charge takes values in an appropriate $\mathrm{K}$-theory group of the space-time $X$ when $X$ is large compared to the string scale. (See [17-19] for subsequent studies.) If this also holds for any size of $X, \mathrm{~T}$-duality should induce an isomorphism of a K-theory group of $T^{n} \times M$ to another K-theory group of the dual $\widehat{T}^{n} \times M$. In fact, brane-anti-brane systems naturally appear in our argument for "Nahm transform = T-duality", and an isomorphism between K-theory groups emerges very naturally. This seems to be actually not limited to four-torus; As another non-trivial application of our argument, we will construct the isomorphism of K-theory groups for T-duality on torus of other dimensions. During the course, we find the identification of the $\mathrm{K}$-theory group for Type II $\mathbf{Z}_{2}$-orientifold: $\mathrm{D}$-brane charges in the presence of an orientifold $p$-plane are classified by $\mathrm{KR}^{-(9-p)}$ or $\mathrm{KR}^{-(5-p)}$ for $S O$ - or $S p$-type orientifold respectively.

This paper is organized as follows. In section 2 , we give a simple ar- 
gument which shows that Nahm-transform can indeed be identified as the transform of D-branes under T-duality on four-torus. We extend the argument to the case where the Nahm transform fails to become a vector bundle and give the interpretation of the resulting object as a certain bound state of D-branes and anti-D-branes. The basic argument of section 2 is applied in section 3 to string theory with orientifold or orbifold projections. This leads to the generalization of Nahm transform to instantons of orthogonal and symplectic gauge groups as well as to instantons on $\mathbf{Z}_{2}$ orbifold. In section 4 , as an application of the picture of brane-anti-brane bound state, we obtain index-theoretic isomorphisms of $\mathrm{K}$-theory groups that realizes $\mathrm{T}$-duality on four-torus. This is extended in section 5 to find isomorphisms of K-theory groups for T-duality on torus of other dimensions. There we also indentify the K-theory group that classifies D-branes in Type II orientifold.

T-duality was discussed in terms of K-theory also in [20,21] which appeared after this paper. The former considers D-branes in algebraic varieties while the latter takes a close look at T-duality in Type II orientifold. They are closely related to sections 2 and 5 respectively.

\section{D-branes on Four-Torus and T-duality}

In this section we study how D-brane configurations are transformed under T-duality on four-torus in Type II string theory.

Let us consider Type II string theory on $\mathbf{R}^{6} \times T^{4}$ with $N \mathrm{D}(p+4)$ branes wrapped on $T^{4}$. We choose flat orthonormal space-time coordinates $x^{M}=x^{0}, \ldots, x^{9}$ and consider $T^{4}$ as $\mathbf{R}^{4} / \Lambda$ where $\mathbf{R}^{4}$ is spanned by $x^{\mu}=x^{1}, x^{2}, x^{3}, x^{4}$ and $\Lambda=\left\{n^{\mu}\right\} \subset \mathbf{R}^{4}$ is a lattice of rank four. The theory contains $U(N)$ super-Yang-Mills theory on the brane with sixteen supercharges. The supersymmetry transformation of the gaugino $\psi$ is given by

$$
\delta \psi=-\frac{1}{4} F_{M N} \Gamma^{M N} \epsilon
$$

where $\epsilon$ is an anticommuting parameter which is a positive chirality spinor in ten dimensions and $F_{M N}$ is the field strength of the (extended) $U(N)$ gauge field. If the gauge field has a self-dual field strength on $T^{4}$ with non-zero instanton number, the supersymmetry is broken to half and only those with positive chirality on $T^{4}$ are unbroken. This is because $F_{\mu \nu} \Gamma^{\mu \nu}=\frac{1}{2} F_{\mu \nu}^{\mp} \Gamma^{\mu \nu}$ 
on spinors with chirality \pm on $T^{4}$ where $F^{ \pm}=F \pm * F .{ }^{1}$ This together with the fact that an instanton on $\mathrm{D}(p+4)$-branes carries a unit charge of the $\mathrm{RR}$ $p$-form potential through the Chern-Simons coupling [22] suggest that the instantons on $T^{4}$ can be identified with $\mathrm{D} p$-branes at points of $T^{4}$. Namely, $N \mathrm{D}(p+4)$-branes with a $k$-instanton configuration on $T^{4}$ can be considered as a classical bound state of $N \mathrm{D}(p+4)$-branes and $k \mathrm{D} p$-branes. ${ }^{2}$ This description of $\mathrm{D} p$-branes as instantons on $\mathrm{D}(p+4)$-branes is of course valid only when the size of the torus is large compared to the string scale.

Now we address the following question: How is T-duality transformation of D-branes described in terms of gauge fields on $T^{4}$ ? We first have to note that T-duality inverts the size of the torus (when B-field is zero) with respect to the string scale. Thus, the question makes sense only when there is a natural one-to-one correspondence between D-brane configurations on large and small torus. In the present case, eight supersymmetries and non-renormalization theorm assures this, since parameters for D-brane configuration and the size of the torus belong to different supermultiplets.

In what follows we provide an answer to this question. We will work in the case $p=5$ since we want to introduce branes of lower dimensions as probes. This means that we work in (unphysical) Type IIB string theory with $N$ D9-branes on $\mathbf{R}^{6} \times T^{4}$. Tadpole, anomaly and other sickness of the theory do not affect our argument which we are going to make. We could also work in the barely physical case of $p=4$ in which most of our argument can be repeated, although it is less convenient compared to $p=5$.

\footnotetext{
${ }^{1}$ In this paper, positive or + (resp. negative or - ) chirality in four dimensions means $\Gamma^{1234}=1$ (resp. -1$)$ (and likewise, in $1+1$ or $5+1$ dimensions (spanning 09 or 012349 directions), it means $\Gamma^{09}$ or $\Gamma^{012349}=1$ (resp. -1$)$ ). This convention in four-dimensions appears to conflict with mathematical notation where in $2 n$-dimension spinor bundles with $i^{n} \Gamma^{12 \cdots 2 n}= \pm 1$ is denoted as $S^{ \pm}$. Nevertheless, we shall follow this mathematical convention as far as the notation $S^{ \pm}$is concerned. Thus, in this paper the bundle of positive (or + ) chirality spinors (with $\Gamma^{1234}=1$ ) is denoted as $S^{-}$whereas $S^{+}$is the bundle of negative (or - ) chirality spinors (with $\Gamma^{1234}=-1$ ). I hope that this does not confuse the reader.

${ }^{2}$ It was noted in [23] that (2.1) admits another term with values in the center of $U(N)$ which relaxes the self-duality condition for supersymmetry. In this section we do not consider the associated generalization which necessarily involves the first Chern class or $\mathrm{D}(p+2)$-branes.
} 


\subsection{Nahm Transform as T-duality}

\section{Probing by a D1-brane}

Let us first probe this D9-brane system by a D1-brane which spans the coordinates $x^{0,9}$, or $x^{ \pm}=x^{0} \pm x^{9}$. The analysis of lowlying spectrum of open strings ending on this probe (in the case where $T^{4}$ is large) is the same as the one for a D1-brane in Type I string theory [24] except that we do not impose invariance under worldsheet orientation reversal. The theory on the probe is a $U(1)$ gauge theory in $1+1$ dimensions with at most $(0,8)$ supersymmetry. It has an $(8,8) U(1)$ vector multiplet whose scalar components take values in $\mathbf{R}^{4} \times T^{4}$ and also a positive-chirality fermion $\lambda$ with $U(1)$ charge -1 which transforms in the fundamental representation of the flavor group $U(N)$. The fermion $\lambda$ is coupled to the $U(N)$ gauge field $A_{\mu} \mathrm{d} x^{\mu}$ on the D9-branes via the minimal coupling

$$
\bar{\lambda}\left(\partial_{-}+\partial_{-} X^{\mu} A_{\mu}(X)-i a_{-}\right) \lambda
$$

where $i a_{ \pm} \mathrm{d} x^{ \pm}$is the $U(1)$ gauge field and $X^{\mu}$ are the scalar fields in the $U(1)$ vector multiplet representing the position of the probe in $T^{4}$. If the gauge field $A_{\mu}$ is flat, the theory preserves the $(0,8)$ supersymmetry (generated by those $\epsilon$ with $\left.\Gamma^{0} \Gamma^{9}=1\right)$ but if it is in an instanton configuration with selfdual curvature, $F_{\mu \nu}^{-}=0$, the supersymmetry is broken to $(0,4)$ (generated by those $\epsilon$ with $\left.\Gamma^{0} \Gamma^{9}=\Gamma^{1} \Gamma^{2} \Gamma^{3} \Gamma^{4}=1\right)$. For a finite size torus, there are actually infinitely many additional modes coming from the strings winding around 1-cycles of $T^{4}$, but the effect of them is small when the size of $T^{4}$ is large and they simply decouple in the low energy limit.

\section{Probing by a Wrapped D5-brane}

Next, let us probe the D9-brane system by a D5-brane wrapped on $T^{4}$ and spanning the $x^{0,9}$ or $x^{ \pm}$directions. The analysis of low-lying modes on the D5-brane is almost identical to that in [25]. The theory of these modes is a $U(1)$ gauge theory in $5+1$ dimensions with at most $(1,0)$ supersymmetry. It has a $(1,1) U(1)$ vector multiplet and a $(1,0)$ hypermultiplet with $U(1)$ charge -1 which transforms in the fundamental representation of the flavor group $U(N)$. The flavor group $U(N)$ has a background gauge field which is given by the gauge field $A_{\mu} \mathrm{d} x^{\mu}$ of the D9-brane. Namely, the hypermultiplet 
fields - scalars $Q^{\sigma}(\sigma=1,2)$ and a negative chirality spinor $\Psi$ - are minimally coupled to $A_{\mu}$ via

$$
\left\|D Q^{\sigma}\right\|^{2}+\bar{\Psi} \not D \Psi
$$

where $D_{ \pm}=\partial_{ \pm}-i a_{ \pm}$and

$$
D_{\mu}=\partial_{\mu}+A_{\mu}-i a_{\mu}
$$

in which $i a_{ \pm} \mathrm{d} x^{ \pm}+i a_{\mu} \mathrm{d} x^{\mu}$ is the $U(1)$ gauge field of the probe. When the $U(N)$ gauge field $A_{\mu} \mathrm{d} x^{\mu}$ is flat, the theory preserves the $(1,0)$ supersymmetry but if it is in an instanton configuration on $T^{4}$ with self-dual curvature $F_{\mu \nu}^{-}=0$, the supersymmetry is broken to half - only those with positivechirality in $T^{4}$ (as well as in $\mathbf{R}^{2} \times T^{4}$ ) is unbroken. We shall consider the case where the instanton number is $k$, namely $A_{\mu}$ is a gauge field that defines a connection of a rank $N$ complex vector bundle $E$ over $T^{4}$ with the second Chern character $\operatorname{ch}_{2}(E)=k$. Then, the wrapped D5-brane is probing a bound state of $N$ D9-branes wrapped on $T^{4}$ and $k$ D5-branes at points in $T^{4}$.

When the size of $T^{4}$ becomes small, it is natural to consider the theory of the wrapped D5-brane probe as $1+1$ dimensional theory with a tower of infinite Kaluza-Klein modes. The theory is invariant under $(0,4)$ supersymmetry which is generated by those $\epsilon$ with $\Gamma^{0} \Gamma^{9}=\Gamma^{1} \Gamma^{2} \Gamma^{3} \Gamma^{4}=1$. At long distances, massive modes simply decouple and only finitely many massless modes remain in the theory. From the six-dimensional $(1,1) U(1)$ vector multiplet, we obtain $(0,4)$ multiplets whose bosonic fields are the $U(1)$ gauge field $a_{ \pm}$together with the scalar fields taking values in the space of solutions to the equations

$$
\begin{aligned}
& \partial_{\mu} X^{p}=0 \\
& \partial_{\mu} a_{\nu}-\partial_{\nu} a_{\mu}=0
\end{aligned}
$$

where $X^{p}(p=5,6,7,8)$ are the scalar component of the $(1,1)$ vector multiplet. The solution space for the first equation is $\mathbf{R}^{4}$ since it is solved by $X^{p}=$ constant. The second equation is the equation for $i a_{\mu} \mathrm{d} x^{\mu}$ to be a flat $U(1)$ connection over $T^{4}$. Since we have a $U(1)$ gauge symmetry, what we actually obtain as the target space is the moduli space of flat $U(1)$ connections. A flat $U(1)$ gauge field can have $a_{\mu}=$ constant, and $a_{\mu}$ is gauge equivalent to $a_{\mu}+\hat{n}_{\mu}$ where $\hat{n}_{\mu}$ belongs to the dual lattice $2 \pi \Lambda^{*}$ of $\Lambda / 2 \pi$ so that the 
gauge transformation $g(x)=\mathrm{e}^{i \hat{n} x}$ is single valued on $T^{4}$. The moduli space is therefore the dual torus $\widehat{T}^{4}=\left\{a_{\mu}\right\} /\left\{\hat{n}_{\mu}\right\}=\left(\mathbf{R}^{4}\right)^{*} / 2 \pi \Lambda^{*}$. Thus, from the six-dimensional $(1,1) U(1)$ vector multiplet, we obtain a $1+1$ dimensional $(8,8) U(1)$ vector multiplet whose scalar components take values in $\mathbf{R}^{4} \times \widehat{T}^{4}$.

Massless fields in $1+1$ dimensions also come from the hypermultiplet in the bifundamental representation and these break the supersymmetry to $(0,4)$. They correspond to the fields on $T^{4}$ satisfying

$$
D_{\mu} Q^{\sigma}=0, \quad \gamma^{\mu} D_{\mu} \psi=0,
$$

where $D_{\mu}$ is given by (2.4). For a generic instanton configuration, there is no covariantly constant section of the associated bundle in the fundamental representation. Therefore, there is no solution to $D_{\mu} Q^{\sigma}=0$. In this case, there is neither positive-chirality Dirac zero mode since such a thing, if existed, would be covariantly constant in a self-dual instanton background as one can see from the Weizenbock formula $\not D^{\dagger} \not D=D^{\dagger} D-F_{\mu \nu} \gamma^{\mu \nu}$. However, as the index theorem shows, there are $k$ Dirac zero modes with negative chirality (see section 4). Let us choose a family of orthonormal basis $\psi_{1}(a), \ldots, \psi_{k}(a)$ of the space of zero modes which varies smoothly as $a_{\mu}$ is varied. Let us expand the field $\Psi$ as

$$
\Psi\left(x^{ \pm}, x^{\mu}\right)=\sum_{i=1}^{k} \psi_{i}\left(a\left(x^{ \pm}\right)\right)\left(x^{\mu}\right) \otimes \lambda^{i}\left(x^{ \pm}\right),
$$

where $\lambda_{i}$ are positive chirality spinors in $1+1$ dimensions (positive because $\Psi$ and $\psi_{i}(a)$ are negative in $5+1$ and 4 -dimensions respectively). Inserting this in the lagrangian (2.3) and integrating over $T^{4}$, we obtain a lagrangian for $\lambda=\left(\lambda^{i}\right)$

$$
\bar{\lambda}\left(\partial_{-}+\partial_{-} a_{\mu} \hat{A}^{\mu}(a)-i a_{-}\right) \lambda
$$

where

$$
\hat{A}^{\mu}(a)_{\bar{\imath} j}=\int_{T^{4}} \mathrm{~d}^{4} x \psi_{i}(a)^{\dagger} \frac{\partial}{\partial a_{\mu}} \psi_{j}(a) .
$$

This $\hat{A}^{\mu}(a)$ can naturally be considered as a $U(k)$ gauge field on the parameter space $\left(\mathbf{R}^{4}\right)^{*}=\left\{a_{\mu}\right\}$ since the choice of orthonormal basis $\psi_{i}(a)$ is arbitrary and can be changed to other ones. Thus, we have obtained from the hypermultiplet a positive chirality fermion $\lambda$ with a unit $U(1)$ charge which is coupled to the external gauge field $\hat{A}^{\mu}(a)$ given by (2.10). In other words, $\lambda$ takes values in the bundle $\hat{E}$ of Dirac zero modes provided with 
the unitary connection $\hat{A}$; the fibre of $\hat{E}$ at $a \in\left(\mathbf{R}^{4}\right)^{*}$ is the kernel of $\not D$ associated with $D_{\mu}=\partial_{\mu}+A_{\mu}-i a_{\mu}$ which is provided with the natural inner product coming from the integration over $T^{4}$. Note that the $U(1)$ gauge transformation $\Psi \rightarrow \mathrm{e}^{i \hat{n} x} \Psi$ sends a zero mode $\psi$ at $a$ to a zero mode $\tilde{\psi}$ at $a+\hat{n}$ defined by $\tilde{\psi}(x)=\mathrm{e}^{i \hat{n} x} \psi(x)$. Thus, $\hat{E}$ should be considered as a bundle over $\left(\mathbf{R}^{4}\right)^{*} / 2 \pi \Lambda^{*}=\widehat{T}^{4}$ where we identify $\psi \equiv \tilde{\psi}$. It is clear that $\hat{A}$ in $(2.10)$ defines a connection on it.

We see that the $1+1$ dimensional theory we obtained is exactly the same as the effective theory of a D1-brane in Type IIB string theory on $\mathbf{R}^{6} \times \widehat{T}^{4}$ which probes D9-branes supporting the bundle $\hat{E}$ with connection $\hat{A}$.

The transform $A \mapsto \hat{A}$ of a gauge field on $T^{4}$ to that of its dual $\widehat{T}^{4}$ is nothing but what is known as the Nahm transform [11-13]. In particular, the $U(k)$ gauge field $\hat{A}^{\mu} \mathrm{d} a_{\mu}$ on $\widehat{T}^{4}$ is actually an instanton with self-dual field strength $\widehat{F}=* \widehat{F}$, as expected from supersymmetry. As we will see in section 4 using the index theorem, the topological numbers of the bundle $\hat{E}$ is related to that of $E$ as $\operatorname{rank}(\hat{E})=\operatorname{ch}_{2}(E)=k$ and $\operatorname{ch}_{2}(\hat{E})=\operatorname{rank}(E)=N$. Thus, the system of D9-branes wrapped on $\widehat{T}^{4}$ supporting $(\hat{E}, \hat{A})$ can be considered as a system of $k$ D9-branes wrapped on $\widehat{T}^{4}$ and $N$ D5-branes at points on $\widehat{T}^{4}$.

To summarize, we have seen that the theory on the wrapped D5-brane probe is effectively the same as the effective theory on a D1-brane moving in $\mathbf{R}^{6} \times \widehat{T}^{4}$ and probing the system of D9-branes which support the gauge field configuration $\hat{A}^{\mu}(a) \mathrm{d} a_{\mu}$ on the dual torus $\widehat{T}^{4}$ given by (2.10) (which represent a system of $k$ D9 and $N$ D5 branes). By definition, the effective theory of a D5-brane wrapped on a small $T^{4}$ is identified as the effective theory of the T-dual D1-brane moving in the large $\widehat{T}^{4}$. Then, the system of $k \mathrm{D} 9$ and $N$ D5 branes emerged above must be identified as T-dual to the original system of $k \mathrm{D} 9$ and $N$ D5 branes. Therefore, what we have seen shows that the the T-duality of D9-D5 brane system (or other (physical) $\mathrm{D}(p+4)$ - $\mathrm{D} p$ brane system with $p=0,1,2,3,4)$ is indeed given by the Nahm transform of instantons on $T^{4}$ and $\widehat{T}^{4}$.

One important property of T-duality is that if the T-duality is operated twice we get back in the same string background. It is indeed known as the inversion theorem $[12,13]$ that the square $\mathrm{Nahm}$ transform is the identity, $(E, A) \mapsto(\hat{E}, \hat{A}) \mapsto(\hat{\hat{E}}, \hat{\hat{A}}) \cong(E, A)$ (see also Appendix A for the expression 
of the isomorphism $(\hat{\hat{E}}, \hat{\hat{A}}) \cong(E, A))$.

Use of brane probe to study space-time geometry was initiated in [26] and has been an important method in string theory. The paper [26] considers D9-D5 system in flat non-compact space-time probed by a D1-brane and obtains ADHM construction of instantons on $\mathbf{R}^{4}$ (which is nothing but the S-dual of the heterotic worldsheet theory in [27]). Since Nahm-transform is in a sense the ADHM construction for four-torus, what we have done can be considered as a generalization of [26]. Indeed, similar argument had been used in [28] where Nahm equation for monopoles on $\mathbf{R}^{3}$ was considered. ${ }^{1}$ Our argument is also close to the one in [31] where D3-branes wrapped on three-torus in a Calabi-Yau three-fold are identified with D0-branes at points in the mirror.

\subsection{The General Case - Emergence of Brane-Anti-Brane System}

In the above discussion, we have assumed that the Dirac operator $\not D=$ $\gamma^{\mu}\left(\partial_{\mu}+A_{\mu}-i a_{\mu}\right)$ has no positive-chirality zero mode at any value of $a_{\mu}$. In general, however, we encounter the cases where there are positive-chirality zero modes as well. This includes the simplest and important example of $k=0$ where the connection $A$ is flat, $A_{\mu}=\operatorname{diag}\left(i a_{\mu}^{1}, \ldots, i a_{\mu}^{N}\right)$, in which there are both positive and negative chirality zero modes at $a_{\mu}=a_{\mu}^{j}$. In such a case, the T-dualized system we obtain is not a vector bundle on $\widehat{T}^{4}$ supported by D9-branes, but something else which we now describe.

\section{Kaluza-Klein Modes and Interpretation}

Let us look at the lagrangian for the full Kaluza-Klein modes from the hypermultiplet of 5-9 strings. The scalar fields $Q^{\sigma}$ can be considered as $1+1$ dimensional scalar fields $q^{\sigma}$ with values in the infinite dimensional vector space $\Gamma(E)$ of sections of $E$. Likewise, the fermion $\Psi$ can be considered to consist of $1+1$ dimensional spinor fields $\lambda_{+}$and $\lambda_{-}$of positive and negative chirality respectively which take values in the infinite dimensional vector

\footnotetext{
${ }^{1}$ Certain monopoles can be considered as instantons on $S^{1} \times \mathbf{R}^{3}$ and [28] is closely related to T-duality on $S^{1}$. Nahm transform on $S^{1}$ (and $T^{2}$ ) is also discussed in $[29,30]$ more completely than [28] but their argument is not applicable to T-duality on $T^{4}$.
} 
spaces $\Gamma\left(S^{+} \otimes E\right)$ and $\Gamma\left(S^{-} \otimes E\right)$. It is appropriate to consider these vector spaces of sections of $E, S^{+} \otimes E$ and $S^{-} \otimes E$ as infinite-dimensional vector bundles $\mathcal{E}, \mathcal{E}^{+}$and $\mathcal{E}^{-}$over $\widehat{T}^{4}$ provided with hermitian metrics and natural connections which we denote by $\mathcal{A}, \mathcal{A}_{+}$and $\mathcal{A}_{-}$respectively: Recall that the $U(1)$ gauge symmetry of the probe D5-brane identifies a section $\psi(x)$ at $a \in\left(\mathbf{R}^{4}\right)^{*}$ and a section $\mathrm{e}^{i \hat{n} x} \psi(x)$ at $a+\hat{n} \in\left(\mathbf{R}^{4}\right)^{*}$ of any of the bundles $E, S^{+} \otimes E$ and $S^{-} \otimes E$. This defines the bundles $\mathcal{E}, \mathcal{E}^{+}$and $\mathcal{E}^{-}$over $\widehat{T}^{4}$. Since this identification is unitary and does not involve $a_{\mu}$ explicitly, the hermitian products and the trivial connections of the trivial bundles over $\left(\mathbf{R}^{4}\right)^{*}$ descends to hermitian products and connections of the bundles over $\widehat{T}^{4}$.

Note that the components $D^{+}: \Gamma\left(S^{+} \otimes E\right) \rightarrow \Gamma\left(S^{-} \otimes E\right)$ and $D^{-}:$ $\Gamma\left(S^{-} \otimes E\right) \rightarrow \Gamma\left(S^{+} \otimes E\right)$ of the Dirac operator $\not D$ are conjugate to each other and that the Weizenbock formula shows $D^{+} D^{-}=-D^{\mu} D_{\mu}$. Then, denoting the operator $D^{+}$at $a$ as $\mathcal{D}(a):\left.\left.\mathcal{E}^{+}\right|_{a} \rightarrow \mathcal{E}^{-}\right|_{a}$, the lagrangian can be written as

$$
\begin{aligned}
& \sum_{\sigma=1,2} \sum_{u=0,9} \mp\left|\left(\partial_{u}+\partial_{u} a_{\mu} \mathcal{A}^{\mu}(a)-i a_{u}\right) q^{\sigma}\right|^{2}+\sum_{\sigma=1,2} \overline{q^{\sigma}} \mathcal{D}(a) \mathcal{D}(a)^{\dagger} q^{\sigma} \\
& +\overline{\lambda_{+}}\left(\partial_{-}+\partial_{-} a_{\mu} \mathcal{A}_{+}^{\mu}(a)-i a_{-}\right) \lambda_{+}+\overline{\lambda_{-}}\left(\partial_{+}+\partial_{+} a_{\mu} \mathcal{A}_{-}^{\mu}(a)-i a_{+}\right) \lambda_{-} \\
& +\overline{\lambda_{-}} \mathcal{D}(a) \lambda_{+}+\overline{\lambda_{+}} \mathcal{D}(a)^{\dagger} \lambda_{-} .
\end{aligned}
$$

We interpret (2.11) as the lagrangian for a D1-brane probing some bound state of infinitely many D9-brane and anti-D9-branes wrapped on $\widehat{T}^{4}$ of the T-dualized system. This is based on the following consideration.

If there are $n$ D9-branes and a D1-brane, the 1-9 strings creates a positivechirality fermion on the $1+1$ dimensional worldvolume of the D1-brane which is charged under the bifundamental representation of $U(n) \times U(1)$. Similarly, if there are $\bar{n}$ anti-D9-brane and a D1-brane, the 1- $\overline{9}$ strings create a negative chirality fermion in the bifundamental of $U(\bar{n}) \times U(1)$. If there are $n$ D9-brane and $\bar{n}$ anti-D9-branes, the analysis of open strings shows that, in addition to the $U(n) \times U(\bar{n})$ gauge fields created by $9-9$ and $\overline{9}-\overline{9}$ strings, there are tachyonic modes created by $9-\overline{9}$ strings which are charged under $U(n) \times U(\bar{n})$ as the bifundamentals $\left(\mathbf{n}, \overline{\mathbf{n}}^{*}\right)$ and $\left(\overline{\mathbf{n}}, \mathbf{n}^{*}\right)$. (In this paper, we denote the dual of a representation $V$ by $V^{*}$.) Therefore, the configuration without the tachyon expectation value is unstable and is expected to roll down toward a minimum of the tachyon potential. Since we do not have a 
correct decription of some stable bound state of a brane-anti-brane system, we do not precisely know what happends for the theory on a D1-brane probing such a system. However, we may expect that the low-lying spectrum is not very much different from the simple superposition of those for the 1-9 system and those for the $1-\overline{9}$ system. In particular, there will be positive and negative chirality fermions in $(\mathbf{n}, \mathbf{1},-1)$ and $(\mathbf{1}, \overline{\mathbf{n}},-1)$ of $U(n) \times U(\bar{n}) \times U(1)$ respectively (and their duals). We may also expect that the tachyon vev will provide a mass term in the theory of D1-brane which couples the positive and negative chirality fermions created by the 1-9 and 1-9 strings. If it is the case, when the tachyon vev is non-zero the coupled fermions are irrelevant in the infra-red. This is consistent with the expectation that a D-brane and an anti-D-brane will annihilate via the tachyon condensation.

Now, part of the interpretation of (2.11) is clear. The fermion $\lambda_{+}$is interpreted as the collection of fermions created by the 1-9 strings in the $n \rightarrow \infty$ limit, whereas $\lambda_{-}$is interpreted as those created by. 1- $\overline{9}$ strings in the $\bar{n} \rightarrow \infty$ limit. The connections $\mathcal{A}_{+}$of $\mathcal{E}^{+}$and $\mathcal{A}_{-}$of $\mathcal{E}^{-}$are the connections of the Chan-Paton bundles supported by the D9 and anti-D9branes respectively. The operators $\mathcal{D}: \mathcal{E}^{+} \rightarrow \mathcal{E}^{-}$and $\mathcal{D}^{\dagger}: \mathcal{E}^{-} \rightarrow \mathcal{E}^{+}$are interpreted as the tachyon fields.

However, it is not obvious how to interpret the scalar fields $q^{\sigma}$ which become massless at the locus where $\mathcal{D}(a)^{\dagger}$ has a zero. Their charge under the symmetry of the system suggest that they are created from the 1-9 or 1-9 $\overline{9}$ strings, but the standard analysis shows that the NS sector of the 1-9 or $1-\overline{9}$ strings has the lowest mass squared $=\frac{1}{2}$ and therefore the corresponding bosons can never become massless. We leave this as an open problem. In this paper, we consider the existence of $q^{\sigma}$ as a consequence of the $(0,4)$ supersymmetry (required from the supersymmetry of the bound state) which postulates the existence of the superpartner of the fermions of mass-squared matrix $\mathcal{D}(a) \mathcal{D}(a)^{\dagger}$.

\section{Localized Degrees of Freedom}

Irrespective of the interpretation as the bound state of D9-branes and antiD9-branes, it is clear that the modes with non-zero eigenvalues of the Laplace/ Dirac operators $-\mathcal{D}(a) \mathcal{D}(a)^{\dagger}$ or $\mathcal{D}(a)^{\dagger} \mathcal{D}(a)$ - are irrelevant in the infra-red limit and can be ignored. When $\mathcal{D}(a)^{\dagger}$ has no kernel everywhere on $\widehat{T}^{4}$, we 
only have to take into account the kernel of $\mathcal{D}(a)$, and we are back in the cases considered in section 2.1: we obtain the Nahm transform $\hat{E}$ supported by $k$ D9-branes of the T-dualized system.

Something special happens when $\mathcal{D}(a)^{\dagger}$ has a non-trivial kernel (and the kernel of $\mathcal{D}(a)$ jumps) at some locus $M$ in $\widehat{T}^{4}$. The mass of some supermultiplet (two complex scalars and two Dirac fermions) goes down toward $M$ and vanish at $M$. In such a case, this multiplet can no longer be ignored at least in a neighborhood of $M$. In other words there are some degrees of freedom localied at $M \subset \widehat{T}^{4}$.

We examine what this is in our favorite example of $k=0$ and flat $A_{\mu}$. For simplicity we consider the case $N=1$. In this case, $E$ is the trivial complex line bundle over $T^{4}$ and the flat connection is given by $A_{\mu}=i a_{\mu}^{0}$ (constant). The Dirac operators are

$$
\mathcal{D}(a)=\bar{\sigma}^{\mu}\left(\partial_{\mu}+i\left(a_{\mu}^{0}-a_{\mu}\right)\right), \quad \mathcal{D}(a)^{\dagger}=\sigma^{\mu}\left(\partial_{\mu}+i\left(a_{\mu}^{0}-a_{\mu}\right)\right),
$$

where $\bar{\sigma}^{\mu}: S^{+} \rightarrow S^{-}$and $\sigma^{\mu}: S^{-} \rightarrow S^{+}$are represented as

$$
\sigma^{1}=\left(\begin{array}{cc}
0 & i \\
i & 0
\end{array}\right), \quad \sigma^{2}=\left(\begin{array}{cc}
0 & 1 \\
-1 & 0
\end{array}\right), \quad \sigma^{3}=\left(\begin{array}{cc}
i & 0 \\
0 & -i
\end{array}\right), \quad \sigma^{4}=\left(\begin{array}{cc}
1 & 0 \\
0 & 1
\end{array}\right),
$$

and $\bar{\sigma}^{\mu}=\sigma^{\mu \dagger}$ under trivializations $S^{+} \cong T^{4} \times \mathbf{C}^{2}$ and $S^{-} \cong T^{4} \times \mathbf{C}^{2}$. There is no kernel for both $\mathcal{D}(a)$ and $\mathcal{D}(a)^{\dagger}$ everywhere except $a=a^{0}$. At $a=a^{0}$, the constant sections of $S^{+} \cong T^{4} \times \mathbf{C}^{2}$ and $S^{-} \cong T^{4} \times \mathbf{C}^{2}$ become the kernels of $\mathcal{D}(a)$ and $\mathcal{D}(a)^{\dagger}$ respectively and there are nothing else. Thus, we only have to look at the spaces $\mathcal{E}_{0}^{+}$and $\mathcal{E}_{0}^{-}$of these constant sections in the vicinity of $a=a^{0}$. The operator $\mathcal{D}(a)$ sends $\mathcal{E}_{0}^{+}$to $\mathcal{E}_{0}^{-}$and behaves as

$$
\mathcal{D}(a)=-i \bar{\sigma}^{\mu}\left(a-a^{0}\right)_{\mu} .
$$

What these all mean in the $1+1$ dimensional theory of the probe is that there are two complex bosons $q_{0}^{\sigma}$ and two positive and negative chirality fermions $\lambda_{+}^{\alpha}, \lambda_{-}^{\dot{\alpha}}$ which are localized near $a=a^{0}$. Their mass squared behaves as $\mathcal{D}(a) \mathcal{D}(a)^{\dagger}=\left|a-a^{0}\right|^{2}$. Note that $q_{0}^{\sigma}$ transforms in the positive spinor representation of $S O(4)$ in the $x^{5678}$ directions while $\lambda_{+}^{\alpha}$ and $\lambda_{-}^{\dot{\alpha}}$ transforms in the negative and positive spinor representations of $S O(4)$ in the $\hat{x}_{1234}$ directions. These are clearly the properties of the 1-5 string in the system of D1-brane probing the D5-brane located at $a=a^{0}$. Thus, this localized 
degrees of freedom can be identified as the D5-brane at $a^{0} \in \widehat{T}^{4}$ of the Tdualized system. This is of course what is expected from the known relation of the Wilson line and the position of the D-branes under T-duality $[1,2]$.

The same conclusion can be deduced also in the picture of D9-anti-D9brane system. Although there are infinitely many D9 and anti-D9-branes, we may regularize the system by discarding infinite high level modes which are irrelevant in the infra-red limit. One obvious choice appears to be the one to discard everything but the constant modes. However, there is a technical difficulty. Because of the identification of the sections $\psi(x)$ at $a$ and $\mathrm{e}^{i \hat{n} x} \psi(x)$ at $a+\hat{n}$, the space of constant sections (defined as $\mathcal{E}_{0}^{ \pm}$in a neighborhood of $a^{0}$ ) does not extend as a globally defined finite-dimensional vector bundle over $\widehat{T}^{4}$. Instead of trying to find a finite-dimensional subbundle of $\mathcal{E}^{ \pm}$, we can consider a more wild but reasonable regularization. Since the only relevant information is the behaviour of the lowest modes in the vicinity of $a=a^{0}$, we can approximate the infinite-dimensional bundles $\mathcal{E}^{ \pm}$with the tachyon $\mathcal{D}: \mathcal{E}^{+} \rightarrow \mathcal{E}^{-}$by rank two vector bundles $\widetilde{\mathcal{E}}_{0}^{ \pm}$with a tachyon $\widetilde{\mathcal{D}}: \widetilde{\mathcal{E}}_{0}^{+} \rightarrow \widetilde{\mathcal{E}}_{0}^{-}$ defined globally over $\widehat{T}^{4}$ such that $\widetilde{\mathcal{D}}: \widetilde{\mathcal{E}}_{0}^{+} \rightarrow \widetilde{\mathcal{E}}_{0}^{-}$is the same as $\mathcal{D}: \mathcal{E}_{0}^{+} \rightarrow \mathcal{E}_{0}^{-}$ when restricted to a neighborhood of $a=a^{0}$ and is an isomorphism outside. Since $\mathcal{D}(a): \mathcal{E}_{0}^{+} \rightarrow \mathcal{E}_{0}^{-}$given by (2.14) has a winding number one on the three-sphere surrounding $a=a^{0}$ and $\widetilde{\mathcal{D}}(a)$ is an isomorphism outside, the instanton numbers of $\widetilde{\mathcal{E}}_{0}^{+}$and $\widetilde{\mathcal{E}}_{0}^{-}$must differ by one, irrespective of the choice of the extension $\widetilde{\mathcal{E}}_{0}^{ \pm}$of $\mathcal{E}_{0}^{ \pm}$. This together with the Chern-Simons coupling on the (anti-)D-brane shows that there is a single D5-brane. Since the degrees of freedom is localized at $a=a^{0}$, the D5-brane must be at $a^{0} \in \widehat{T}^{4}$.

\section{Mukai's Fourier Transform}

We have described what we obtain as the T-dualized system in the general case, using the effective theory on the probe. We have also given an interpretation as some bound state of D9-branes and anti-D9-branes. However, we have not described it in a mathematical language except for the generic case where the kernel of $\mathcal{D}(a)^{\dagger}$ is constantly zero and the T-duality is described as the Nahm transform. In algebraic geometry, there is a transform called Mukai's Fourier transform [10] which can be considered as a generalization of the Nahm transform. It is a transform of an object in a category called derived category of sheaves [32] on an abelian variety $X$ (a complex torus 
embedded in a projective space) denoted by $\mathbf{D}(X)$ to an object of $\mathbf{D}(\widehat{X})$ where $\widehat{X}$ is the dual torus of $X$ (which is again an abelian variety). The category $\mathbf{D}(X)$ includes as its objects holomorphic vector bundles on $X$, and for the case where $X$ is four-torus and in a region where there is a one to one correspondence between holomorphic bundles and (anti-)self-dual connections, Mukai's Fourier transform agrees with the Nahm transform. This suggests that the correct mathematical language to describe what we have obtained should not be far from the derived category of sheaves, at least in the case where $T^{4}$ has a structure of an abelian variety. Indeed, a general object of $\mathbf{D}(X)$ is a complex of sheaves which is reminiscent of our Dirac complex, and also, sheaves can localize on a subvariety of $X$. It is an interesting problem to find the precise relation (though we do not attempt to solve here).

\section{The Inverse Transform}

We have started from a connection of a vector bundle on $T^{4}$ supported by D9-branes and obtained, as its T-dual image, an object which is something more general than a connection of a vector bundle, presumably supported by D9 and anti-D9-branes. Then, a natural question is what happens when T-duality is applied to such an object. To test the interpretation as the D9-anti-D9 bound state, assuming some property of branes probing such a system, we consider the T-duality of the object which is obtained as the Tdual image of our favorite example of $k=0, N=1$ flat connection $A_{\mu}=i a_{\mu}^{0}$. We must get back the original flat connection. We will work in the finitedimensional approximation of $\mathcal{D}: \mathcal{E}^{+} \rightarrow \mathcal{E}^{-}$introduced before and will focus our attention to a neigorhood of $a=a^{0}$, ignoring the global issue on $\widehat{T}^{4}$ which yields only a subleading modification in the present discussion.

Thus, our starting point is the two pairs of D9 and anti-D9-branes wrapped on $\widehat{T}^{4}$ supporting the rank-two Chan-Paton bundles $E^{+}$and $E^{-}$ and the tachyon field $T: E^{+} \rightarrow E^{-}$which behaves near $a=a^{0}$ as (2.14), $T(a)=-i \bar{\sigma}^{\mu}\left(a-a^{0}\right)_{\mu}$. We probe this system by a D5-brane wrapped on $\widehat{T}^{4}$. As in the case for a D1-brane probing the brane-anti-brane system, we assume that the low-lying fermionic spectrum on the D5-brane is the same as the superposition of those for the D5-D9 system and the D5-anti-D9 system. Also, we assume that the tachyon expectation value yields the fermion mass 
term. Thus, the probe theory has $5+1$ dimensional negative and positive chirality fermions with values in $E^{+}$and $E^{-}$which we denote by $\Psi_{-}$and $\Psi_{+}$respectively. The lagrangian for these fields is

$$
\bar{\Psi}_{-} \not D^{-} \Psi_{-}+\bar{\Psi}_{+} \not D^{+} \Psi_{+}+\bar{\Psi}_{+} T \Psi_{-}+\bar{\Psi}_{-} T^{\dagger} \Psi_{+}
$$

where $\not D^{\mp}$ are the $5+1$ dimensional Dirac operator associated with the covariant derivatives $D_{ \pm}=\partial_{ \pm}-i a_{ \pm}$and

$$
D^{\mu}=\frac{\partial}{\partial a_{\mu}}-i x^{\mu}
$$

where $x^{\mu}$ stands for the flat $U(1)$ gauge field on $\widehat{T}^{4}$. Here we have ignored the gauge fields of the bundles $E^{ \pm}$.

Massless fermions in the reduced $1+1$ dimensions would come from the solutions of the equations of motion on the $\widehat{T}^{4}$ factor

$$
\left(\begin{array}{cc}
T & \hat{D}^{-} \\
\hat{D}^{+} & T^{\dagger}
\end{array}\right)\left(\begin{array}{c}
\psi_{++} \\
\psi_{--}
\end{array}\right)=0, \quad\left(\begin{array}{cc}
T & \hat{D}^{+} \\
\hat{D}^{-} & T^{\dagger}
\end{array}\right)\left(\begin{array}{c}
\psi_{-+} \\
\psi_{+-}
\end{array}\right)=0,
$$

for $\psi_{++} \in \Gamma\left(\hat{S}^{+} \otimes E^{+}\right), \psi_{-+} \in \Gamma\left(\hat{S}^{-} \otimes E^{+}\right)$etc, where $\hat{S}^{ \pm}$are negative and postitive spinor bundles on $\widehat{T}^{4}$ and $\hat{D}^{ \pm}$are the components of the Dirac operator associated with $D^{\mu}$. A solution to the equation on the left (resp. right) would lead to a positive (resp. negative) chirality fermion in $1+1$ dimensions.

Now let us take a closer look at these equations. We first recall some facts about the spinor representations in four-dimensions. Let $\mathbf{2}_{ \pm}$be the spinor representation of $\operatorname{Spin}(4)=S U(2)_{+} \times S U(2)_{-}$which are sent to each other by the gamma matrices $\bar{\sigma}^{\mu}: \mathbf{2}_{+} \rightarrow \mathbf{2}_{-}$and $\sigma^{\mu}: \mathbf{2}_{-} \rightarrow \mathbf{2}_{+}$. We note that there are isomorphisms $\epsilon_{ \pm}: \mathbf{2}_{ \pm} \rightarrow \mathbf{2}_{ \pm}^{*}$ of $S U(2)_{ \pm}$representations such that $\epsilon_{-} \bar{\sigma}^{\mu}={ }^{t} \sigma^{\mu} \epsilon_{+}$. Now, using the metric we identify the tangent spaces of $T^{4}$ and $\widehat{T}^{4}$, and thus we can consider both $S^{ \pm}$and $\hat{S}^{ \pm}$as the trivial bundles with the common fibre $\mathbf{2}_{ \pm}$. In particular, the Dirac operators on $\widehat{T}^{4}$ can be respresented as $\hat{D}^{+}=\bar{\sigma}^{\mu} D^{\mu}$ and $\hat{D}^{-}=\sigma^{\mu} D^{\mu}$. On the other hand, the bundles $E^{ \pm}$look like the bundles with fibres $2_{ \pm}$in the vicinity of $a=a^{0}$ where the tachyon is given by $T(a)=-i \bar{\sigma}^{\mu}\left(a-a^{0}\right)_{\mu}$. For convenience, using the isomorphisms $\epsilon_{ \pm}$, we shall consider $E^{ \pm}$to have fibres $\mathbf{2}_{ \pm}^{*}$ and the tachyon is represented as

$$
T(a)=-i^{t} \sigma^{\mu}\left(a-a^{0}\right)_{\mu}
$$


Then, we can consider $\psi_{++}, \psi_{--}, \psi_{-+}$and $\psi_{+-}$as functions taking values in $\mathbf{2}_{+} \otimes \mathbf{2}_{+}^{*}, \mathbf{2}_{-} \otimes \mathbf{2}_{-}^{*}, \mathbf{2}_{-} \otimes \mathbf{2}_{+}^{*}$ and $\mathbf{2}_{+} \otimes \mathbf{2}_{-}^{*}$ respectively, and the equations (2.17) look like

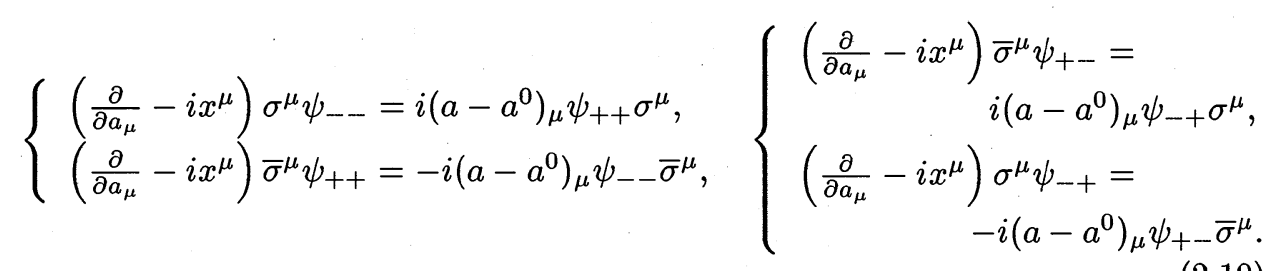

The equations on the left are solved by

$$
\begin{aligned}
& \psi_{++}=1_{\mathbf{2}_{+}} \exp \left(-\frac{1}{2}\left|a-a^{0}\right|^{2}+i x a\right), \\
& \psi_{--}=i 1_{\mathbf{2}_{-}} \exp \left(-\frac{1}{2}\left|a-a^{0}\right|^{2}+i x a\right),
\end{aligned}
$$

while the equations on the right have no solution. This is true for any values of $x^{\mu} \in \mathbf{R}^{4}$. Note that this solution is single valued as a function of $x_{\mu} \in T^{4}$ since it obeys the correct periodicity with respect to $x^{\mu} \rightarrow x^{\mu}+n^{\mu}(n \in \Lambda)$ :

$$
\psi(a) \text { at } x \equiv \mathrm{e}^{i n a} \psi(a) \text { at } x+n
$$

which is dictated by the $U(1)$ gauge symmetry of the probe D5-brane.

Thus, we have a single positive-chirality fermion in the effective $1+1$ dimensional theory. This is interpreted as the 1-9 string mode of the D1brane probing a D9-brane. Since the above solution for $\left(\psi_{++}, \psi_{--}\right)$is single valued and nonwhere vanishing over the whole space $T^{4}$, the Chan-Paton bundle of the D9-brane is topologically trivial. The gauge field on this trivial bundle can be computed as

$$
A_{\mu}(x)=\int_{\widehat{T}^{4}} \mathrm{~d}^{4} a\left(\psi_{++}^{\dagger}, \psi_{--}^{\dagger}\right) \frac{\partial}{\partial x^{\mu}}\left(\begin{array}{c}
\psi_{++} \\
\psi_{--}
\end{array}\right)=i a_{\mu}^{0}
$$

when $\psi_{++}$and $\psi_{--}$are correctly normalized. Thus, we have recovered the original flat connection supported by a single D9-brane.

\section{Orientifold and Orbifold}

We apply the argument of the previous section to the case where the fourtorus is at an orientifold fixed plane or is modded out by $\mathbf{Z}_{2}$ orbifold action. 
We describe the T-duality in terms of the gauge field configuration representing the system. This will lead us to find a $\mathrm{Nahm}$ transform for instantons on $T^{4}$ with orthogonal/symplectic gauge groups or on orbifold $T^{4} / \mathbf{Z}_{2}$. Here we only consider the generic case where the transformed object is a vector bundle, and more general case will not be presented since that would be a repetition of section 2.2. (However, the general case will be included in section 4.)

\subsection{D-branes Wrapped on $\mathrm{Z}_{2}$ Orientifold/Orbifold of Four- Torus}

As preliminaries, we provide the gauge theory description of D-branes wrapped on $\mathbf{Z}_{2}$ orientifold/orbifold of four-torus where $\mathbf{Z}_{2}$ acts on the torus via the inversion - the sign flip of all four flat coordinates. (For orientifold, we consider here the case where D-branes and orientifold-planes are parallel except the four-torus directions.) We apply the method of [33] to find the description. Similar analysis for orientifold has been done in $[34,35]$ and most close one is in [36], while the orbifold case was analyzed in [37,38].

\section{(i) Orientifold of $S O$-Type}

We first consider the case of orientifold of $S O$-type. Thus, we would like to find a gauge theory description of, say, $2 k$ D4-branes in Type IIA orientifold on $\mathbf{R} \times\left(\widehat{T}^{4} \times \mathbf{R}^{5}\right) / \mathbf{Z}_{2}$ which are wrapped on the $\widehat{T}^{4}$ directions. For this, we start with the T-dualized system of $2 k$ D0-branes in Type IIA orientifold on $\mathbf{R} \times T^{4} \times \mathbf{R}^{5} / \mathbf{Z}_{2}$ and perform the Fourier transform as in [33].

If $T^{4}$ were $\mathbf{R}^{4}$, the theory on the D0-branes would be the supersymmetric quantum mechanics with eight supercharges which can be obtained as the dimensional reduction of four-dimensional $N=2 S p(k)$ gauge theory with a hypermultiplet in the anti-symmetric representation. The bosonic fields in such a theory is $S p(k)$ gauge field $A_{0}$ (which can be gauged away), the scalars $X^{p}(p=5,6,7,8,9)$ in the adjoint representation, and the scalars $X^{\mu}$ $(\mu=1,2,3,4)$ in the anti-symmetric representation. The adjoint and antisymmetric representation fields, $X^{p}$ and $X^{\mu}$, can be represented by $2 k \times 2 k$ 
anti-hermitian and hermitian matrices respectively, both of which obey

$$
J \bar{X}=X J, \quad J=\left(\begin{array}{cc}
0 & -\mathbf{1}_{k} \\
\mathbf{1}_{k} & 0
\end{array}\right),
$$

where $\bar{X}$ is the complex conjugation of $X$ and $\mathbf{1}_{k}$ is the $k \times k$ unit matix.

For $T^{4}=\mathbf{R}^{4} / \Lambda$, we must take into accout the open strings winding around 1-cycles (and ending on the D0-branes). Thus, as in [33], we must replace the $S p(k)$ gauge group to the symplectic group " $S p(k|\Lambda|)$ " of infinite rank. The bosonic fields in the theory can be represented by $2 k \times 2 k$ matrices $X_{n m}^{p}, X_{n m}^{\mu}$ parametrized by $(n, m) \in \Lambda \times \Lambda$ which obey $X_{n m}^{p \dagger}+X_{m n}^{p}=$ $0, X_{n m}^{\mu \dagger}=X_{m n}^{\mu}$ and also (3.1) for each $X=X_{n m}^{p}, X_{n m}^{\mu}$. There are also periodicity conditions $X_{\left(n+n^{\prime}\right)\left(m+n^{\prime}\right)}^{p}=X_{n m}^{p}$ and $X_{\left(n+n^{\prime}\right)\left(m+n^{\prime}\right)}^{\mu}=X_{n m}^{\mu}+$ $\left(n^{\prime}\right)^{\mu} \delta_{n, m} \mathbf{1}_{2 k}$. Let us put

$$
\begin{aligned}
& X^{p}(\hat{x})=\sum_{n \in \Lambda} \mathrm{e}^{i n \hat{x}} X_{n 0}^{p} \\
& A^{\mu}(\hat{x})=i \sum_{n \in \Lambda} \mathrm{e}^{i n \hat{x}} X_{n 0}^{\mu}
\end{aligned}
$$

where $\hat{x}=\left(\hat{x}_{\mu}\right)$ are coordinates of the dual torus $\widehat{T}^{4}=\left(\mathbf{R}^{4}\right)^{*} / 2 \pi \Lambda^{*}$. Then, these are functions on $\widehat{T}^{4}$ with values in $2 k \times 2 k$ anti-hermitian matrices and satisfy

$$
\begin{aligned}
& J \overline{X^{p}(\hat{x})}=X^{p}(-\hat{x}) J, \\
& J \overline{A^{\mu}(\hat{x})}=-A^{\mu}(-\hat{x}) J .
\end{aligned}
$$

The $S p(k|\Lambda|)$ gauge transformation is represented as the transformation of the fields $X^{p}(\hat{x}), A^{\mu}(\hat{x})$ given infinitesimally by $\delta X^{p}(\hat{x})=\left[X^{p}(\hat{x}), \alpha(\hat{x})\right]$ and $\delta A^{\mu}(\hat{x})=\left[A^{\mu}(\hat{x}), \alpha(\hat{x})\right]+\partial^{\mu} \alpha(\hat{x})$ where $\alpha(\hat{x})$ is a function on $\widehat{T}^{4}$ which satisfy the same conditions as $X^{p}(\hat{x})$. The lagrangian is the same as the one for the $4+1$ dimensional $N=2$ supersymmetric (sixteen supercharges) $U(2 k)$ Yang-Mills theory formulated on $\mathbf{R} \times \widehat{T}^{4}$. The number of unbroken supersymmetries are reduced to eight by the constraints (3.4) and (3.5) on the fields.

This is the gauge theory description of wrapped $\mathrm{D} 4$ branes in the Type IIA orthogonal-orientifold on $\mathbf{R} \times\left(\widehat{T}^{4} \times \mathbf{R}^{5}\right) / \mathbf{Z}_{2}$. It is obvious how to generalize this to the system of wrapped $\mathrm{D}(p+4)$-branes in the Type II orientifold on $\mathbf{R}^{p+1} \times\left(\widehat{T}^{4} \times \mathbf{R}^{5-p}\right) / \mathbf{Z}_{2}$ with Op-plane of $S O$-type at each of the sixteen fixed point. 
The conditions (3.4) and (3.5) can be restated in a way which applies also to the case where the gauge bundle on $\widehat{T}^{4}$ is topologically non-trivial. Let $E$ be a $U(2 k)$ bundle over $\widehat{T}^{4}$ (a rank $2 k$ complex vector bundle provided with a hermitian fibre metric $()$,$) . The fields X^{p}(\hat{x})$ and $A^{\mu}(\hat{x})$ represent respectively a section $X^{p}$ of the adjoint bundle of $E$ (an anti-hermitian endomorphism of $E$ ) and a unitary connection $\nabla$ of $E$ (a connection preserving the hermitian metric). Now suppose we have a family of anti-liner maps $J=\left(J_{\hat{x}}\right)$ sending the fibre at $\hat{x}$ to the fibre at $-\hat{x}$ :

$$
J_{\hat{x}}: E_{\hat{x}} \longrightarrow E_{-\hat{x}}, \quad \text { such that } J_{-\hat{x}} J_{\hat{x}}=-\mathrm{id}_{\hat{x}}
$$

which is isometric in the sense that $\left(J_{\hat{x}} v, J_{\hat{x}} w\right)=(w, v)$. We shall call such a family $J$ a symplectic structure of $E$ over the inversion $\hat{x} \mapsto-\hat{x}$ of $\widehat{T}^{4}$, and such a pair $(E, J)$ a symplectic orientibundle over the (orthogonal) orientifold $\widehat{T}^{4} / \mathbf{Z}_{2}$. Such anti-linear maps $J$ can be locally represented by the matrix $\left(J_{j i}\right)$ in (3.1) as $J_{\hat{x}} e_{i}(\hat{x})=e_{j}^{\prime}(-\hat{x}) J_{j i}$ where $e_{i}$ is an orthonormal frame of $E$ defined in an open subset of $\widehat{T}^{4}$ and $e_{j}^{\prime}$ is another one defined in the inversion image (we shall call such a pair of frames a symplectic frame). Then, the condition (3.4) means that $X^{p}$ commutes with $J, J_{\hat{x}} X^{p}(\hat{x})=$ $X^{p}(-\hat{x}) J_{\hat{x}}$, and the condition (3.5) requires that the connection $\nabla$ preserves the symplectic structure $J$ in the sense that $J \nabla_{X} s=\nabla_{(-X)} J s$ for a (local) section $s$ and a vector field $X$ on $\widehat{T}^{4}$ where $J s$ is another section defined by $J_{\hat{x}} s(\hat{x})=(J s)(-\hat{x})$ and $-X$ is the image of $X$ under the inversion $\hat{x} \mapsto-\hat{x}$. The gauge transformations are (local) unitary automorphisms of $E$ which commute with the map $J .^{1}$

One can show that a $U(2 k)$ bundle on $\widehat{T}^{4}$ with arbitrary instanton number $N$ admits a symplectic structure over the inversion. Let us choose a neighborhood $D$ of $\hat{x}=0$ given by $|\hat{x}| \leq \epsilon$ and let $\overline{\widehat{T}^{4} \backslash D}$ be the outside region. A $U(2 k)$ bundle $E$ of instanton number $N$ can be constructed by glueing the trivial bundles over $D$ and $\widehat{T}^{4} \backslash D$ at the boundary $S^{3}=\{|\hat{x}|=\epsilon\}$ by a transition function $g: S^{3} \rightarrow U(2 k)$ of winding number $N$. We define the anti-linear map $J_{\hat{x}}: E_{\hat{x}} \rightarrow E_{-\hat{x}}$ over $\widehat{T}^{4} \backslash D$ as the complex conjugation followed by the multiplication by the matrix $J$ in (3.1) with respect to the trivialization we started with. At the boundary $S^{3}$, the matrix to be multiplied is

\footnotetext{
${ }^{1}$ There is another way to state the conditions: Let us define a bilinear pairing $\langle$,$\rangle of$ the fibres at $\hat{x}$ and $-\hat{x}$ by $\left\langle v_{\hat{x}}, w_{-\hat{x}}\right\rangle=\left(J v_{\hat{x}}, w_{-\hat{x}}\right)$. Then, this is a non-degenerate skewsymmetric form (skew-symmetric in the sense that $\left.\left\langle v_{\hat{x}}, w_{-\hat{x}}\right\rangle=-\left\langle w_{-\hat{x}}, v_{\hat{x}}\right\rangle\right)$. Then, the conditions (3.4) and (3.5) means that the gauge transformations and the connection $\nabla$ should preserve the skew-form $\langle$,$\rangle .$
} 
expressed as $J(\hat{x})=g(-\hat{x})^{-1} J \overline{g(\hat{x})}$ with respect to the trivialization that extends over $D$. We can extend $J_{\hat{x}}$ to the interior of $D$ if $J(\hat{x})$ can be extended to a function on $D$ satisfying $J(-\hat{x}) \overline{J(\hat{x})}=-1$ and $J(\hat{x})^{\dagger} J(\hat{x})=1$. Let us embed $S p(1)$ in $S p(k) \subset U(2 k)$ via $h \in S p(1) \mapsto \operatorname{diag}(h, 1, \ldots, 1) \in S p(k)$. Then, a map $\hat{x} \in S^{3}=S p(1) \mapsto \hat{x}^{N} \in S p(1)$ induces a map $g: S^{3} \rightarrow U(2 k)$ of winding number $N$ which yields $J(\hat{x})=(-1)^{N} J$ on $S^{3}$. Thus, $J_{\hat{x}}$ extends to the interior of $D$ and hence to all over $\widehat{T}^{4}$, defining a symplectic structure over the inversion.

\section{(ii) Orientifold of $S p$-Type}

We next consider the case of orientifold of $S p$-type. We shall find a gauge theory description of $k$ D4-branes in Type IIA orientifold on $\mathbf{R} \times\left(\widehat{T}^{4} \times \mathbf{R}^{5}\right) / \mathbf{Z}_{2}$ which are wrapped on the $\widehat{T}^{4}$ directions. We start with the T-dualized system of $k$ D0-branes in Type IIA orientifold on $\mathbf{R} \times T^{4} \times \mathbf{R}^{5} / \mathbf{Z}_{2}$ and proceed as in the previous case. The differece is that (in the case where $T^{4}$ is replaced by $\mathbf{R}^{4}$ ) the gauge group is now $O(k)$ and the hypermultiplet is in the second rank symmetric tensor representation. The effect is to eliminate the matrix $J$ in every formula in the previous case.

Thus, the theory of D4-branes wrapped on $\widehat{T}^{4} / \mathbf{Z}_{2}$ orientifold contains bosonic fields $X^{p}(\hat{x})(p=5,6,7,8,9)$ and $A^{\mu}(\hat{x})(\mu=1,2,3,4)$ with values in $k \times k$ anti-hermitian matrices obeying

$$
\begin{aligned}
& \overline{X^{p}(\hat{x})}=X^{p}(-\hat{x}), \\
& \overline{A^{\mu}(\hat{x})}=-A^{\mu}(-\hat{x}) .
\end{aligned}
$$

The infinitesimal gauge transformation parameter $\alpha(\hat{x})$ obey the same conditions as $X^{p}(\hat{x})$. The lagrangian is the same as the one for $4+1$ dimensional $N=2 U(k)$ super-Yang-Mills theory on $\mathbf{R} \times \widehat{T}^{4}$ but the supersymmetries are reduced to eight by the constarints on the fields.

We restate the conditions (3.7) and (3.8) in a general set up. Let $E$ be a $U(k)$ bundle over $\widehat{T}^{4}$. The fields $X^{p}(\hat{x})$ and $A^{\mu}(\hat{x})$ represent respectively a section $X^{p}$ of the adjoint bundle of $E$ and a unitary connection $\nabla$ of $E$. Now suppose we have a family of anti-liner maps $I=\left(I_{\hat{x}}\right)$ sending the fibre at $\hat{x}$ to the fibre at $-\hat{x}$ :

$$
I_{\hat{x}}: E_{\hat{x}} \longrightarrow E_{-\hat{x}}, \quad \text { such that } I_{-\hat{x}} I_{\hat{x}}=\mathrm{id}_{\hat{x}}
$$


which is isometric in the sense that $\left(I_{\hat{x}} v, I_{\hat{x}} w\right)=(w, v)$. We shall call such a family $I$ an orthogonal structure of $E$ over the inversion $\hat{x} \rightarrow-\hat{x}$ of $\widehat{T}^{4}$, and such a pair $(E, I)$ an orthogonal orientibundle over the (symplectic) orientifold $\widehat{T}^{4} / \mathbf{Z}_{2}$. Such anti-linear maps $I$ can be locally represented as $I_{\hat{x}} e_{i}(\hat{x})=e_{i}^{\prime}(-\hat{x})$ where $e_{i}$ is an orthonormal frame of $E$ defined in an open subset of $\widehat{T}^{4}$ and $e_{j}^{\prime}$ is another one defined in the inversion image (we shall call such a pair of frames a real frame). Then, the condition (3.7) means that $X^{p}$ commutes with $I$ and the condition (3.8) requires that the connection $\nabla$ preserves the symplectic structure $I$ (in the similar sense as before). The gauge transformations are (local) unitary automorphisms of $E$ which commute with the map $I .{ }^{2}$

One can show that a $U(k)$ bundle on $\widehat{T}^{4}$ admits an orthogonal structure over the inversion provided the instanton number is even, say $2 N$. The construction is as in the previous case. Thus, we only have to show that the function $I(\hat{x})=g(-\hat{x})^{-1} \overline{g(\hat{x})}$ on $S^{3}=\{|\hat{x}|=\epsilon\}$ extends to a function defined on $|\hat{x}| \leq \epsilon$ obeying $I(-\hat{x}) \overline{I(\hat{x})}=1$ and $I(\hat{x})^{\dagger} I(\hat{x})=1$ if $g: S^{3} \rightarrow U(k)$ is a map of even winding number $2 N$. Let us embed $S p(1)$ in $S O(k) \subset U(k)$ via $h \in S p(1) \mapsto[(h, 1)] \in(S p(1) \times S p(1)) / \mathbf{Z}_{2}=S O(4) \subset S O(k)$. Then, a map $\hat{x} \in S^{3}=S p(1) \mapsto \hat{x}^{N} \in S p(1)$ induces a map $g: S^{3} \rightarrow U(k)$ of winding number $2 N$. This yields $I(\hat{x})=(-1)^{N}$ which obviously extends to $|\hat{x}| \leq \epsilon$. Note that this construction applies only to the case of even instanton numbers (presumably there is no orthogonal structure over the inversion for odd instanton numbers because of $\left.\pi_{3}(U(k) / O(k))=\mathbf{Z}_{2}\right)$.

\section{(iii) Orbifold}

Finally, we provide a gauge theory description of D-branes wrapped on the orbifold $^{3} T^{4} / \mathbf{Z}_{2}$. We consider here $2 N$ D4-branes (the generalization to $p>4$ branes is obvious).

We start with Type IIA string theory on the dual $\widehat{T}^{4} / \mathbf{Z}_{2}$ with $2 N$ D0-

\footnotetext{
${ }^{2} \mathrm{~A}$ bilinear pairing $\langle$,$\rangle of the fibres at \hat{x}$ and $-\hat{x}$ defined by $\left\langle v_{\hat{x}}, w_{-\hat{x}}\right\rangle=\left(J v_{\hat{x}}, w_{-\hat{x}}\right)$ is a non-degenerate symmetric form (symmetric in the sense that $\left\langle v_{\hat{x}}, w_{-\hat{x}}\right\rangle=\left\langle w_{-\hat{x}}, v_{\hat{x}}\right\rangle$ ). Then, the conditions (3.7) and (3.8) means that the gauge transformations and the connection $\nabla$ should preserve the form $\langle$,$\rangle .$

${ }^{3}$ Here, we consider string theory based on the orbifold CFT on the worldsheet. When $T^{4} / \mathbf{Z}_{2}$ is considered as a singular K3-surafce, NS-NS $B$-field has period $\pi$ for each of the sixteen vanishing two-cycles.
} 
branes at points of $\widehat{T}^{4}$, and proceed as before following [33]. The bosonic fields in the theory of lowlying open string modes can be represented by $2 N \times$ $2 N$ matrices $X_{\hat{n} \hat{m}}^{p}, \hat{X}_{\mu, \hat{n} \hat{m}}$ parametrized by $(\hat{n}, \hat{m}) \in 2 \pi \Lambda^{*} \times 2 \pi \Lambda^{*}$ which obey the usual conditions $X_{\hat{n} \hat{m}}^{p \dagger}+X_{\hat{m} \hat{n}}^{p}=0, \hat{X}_{\mu, \hat{n} \hat{m}}^{\dagger}=\hat{X}_{\mu, \hat{m} \hat{n}}, X_{\left(\hat{n}+\hat{n}^{\prime}\right)\left(\hat{m}+\hat{n}^{\prime}\right)}^{p}=X_{\hat{n} \hat{m}}^{p}$ and $\hat{X}_{\mu,\left(\hat{n}+\hat{n}^{\prime}\right)\left(\hat{m}+\hat{n}^{\prime}\right)}=\hat{X}_{\mu, \hat{n} \hat{m}}+\left(\hat{n}^{\prime}\right)_{\mu} \delta_{\hat{n}, \hat{m}} \mathbf{1}_{2 N}$. The $\mathbf{Z}_{2}$ orbifold projection amounts to $\Phi X_{\hat{n} \hat{m}}^{p} \Phi=X_{-\hat{n}-\hat{m}}^{p}, \Phi \hat{X}_{\mu, \hat{n} \hat{m}} \Phi=-\hat{X}_{\mu,-\hat{n}-\hat{m}}$, where

$$
\Phi=\left(\begin{array}{cc}
0 & \mathbf{1}_{N} \\
\mathbf{1}_{N} & 0
\end{array}\right) .
$$

Here we assumed that the $\mathbf{Z}_{2}$ orbifold action on Chan-Paton factor is in a sum of copies of the regular representation, but we can relax this condition so that $\Phi$ is an arbitrary unitary matrix that squares to 1 . Such matrices are classified by $\operatorname{Tr} \Phi$ up to similarity transformation. ${ }^{4}$ Let us put

$$
\begin{aligned}
& X^{p}(x)=\sum_{\hat{n} \in 2 \pi \Lambda^{*}} \mathrm{e}^{i \hat{n} x} X_{\hat{n} \hat{0}}^{p} \\
& A_{\mu}(x)=i \sum_{\hat{n} \in 2 \pi \Lambda^{*}} \mathrm{e}^{i \hat{n} x} \hat{X}_{\mu, \hat{n} \hat{0}}
\end{aligned}
$$

where $x=\left(x^{\mu}\right)$ are coordinates of the torus $T^{4}$. Then, these are functions on $T^{4}$ with values in $2 N \times 2 N$ anti-hermitian matrices and satisfy

$$
\begin{aligned}
& \Phi X^{p}(x) \Phi=X^{p}(-x) \\
& \Phi A_{\mu}(x) \Phi=-A_{\mu}(-x) .
\end{aligned}
$$

The gauge transformation is represented infinitesimally by $\delta X^{p}(x)=\left[X^{p}(x)\right.$, $\alpha(x)]$ and $\delta A_{\mu}(x)=\left[A_{\mu}(x), \alpha(x)\right]+\partial_{\mu} \alpha(x)$ where $\alpha(x)$ is a function on $T^{4}$ which satisfy the same conditions as $X^{p}(x)$. The lagrangian is the same as the one for the $4+1$ dimensional $N=2 U(2 N)$ super-Yang-Mills theory on $\mathbf{R} \times T^{4}$, but the number of supersymmetries are reduced to eight by the constraints on the fields.

We restate the conditions (3.13) and (3.14) in a general set up where the gauge bundle on $T^{4}$ is not necessarily topologically trivial. Let $E$ be a $U(2 k)$ bundle over $T^{4}$. The fields $X^{p}(x)$ and $A_{\mu}(x)$ represent respectively a section $X^{p}$ of the adjoint bundle and a unitary connection $\nabla$. Now suppose we have a family of linear maps $\varphi=\left(\varphi_{x}\right)$ sending the fibre at $x$ to the fibre at $-x$ :

$$
\varphi_{x}: E_{x} \longrightarrow E_{-x}, \quad \text { such that } \varphi_{-x} \varphi_{x}=\mathrm{id}_{x}
$$

\footnotetext{
${ }^{4}$ If $\operatorname{Tr} \Phi \neq 0$, there must be a fractional brane stuck at the $\mathbf{Z}_{2}$ fixed point [9].
} 
which is unitary in the sense that $\left(\varphi_{x} v, \varphi_{x} w\right)=(v, w)$. We shall call such a family $\varphi$ a lift to $E$ of the inversion $x \mapsto-x$ of $T^{4}$, and such a pair $(E, \varphi)$ an orbibundle over the orbifold $T^{4} / \mathbf{Z}_{2} \cdot{ }^{5}$ Then, the condition (3.13) means that $X^{p}$ commutes with $\varphi$ and the condition (3.14) requires that the connection $\nabla$ preserves $\varphi$ in the usual sense. The gauge transformations are (local) unitary automorphisms of $E$ which commute with the map $\varphi$. Two lifts of the inversion cannot be equivalent if the traces are different at any of the sixteen fixed points $\left\{x_{i}\right\}$. We shall call a lift $\varphi$ traceless if $\operatorname{Tr} \varphi_{x_{i}}=0$ at all of the fixed points.

One can show that a $U(2 N)$ bundle on $T^{4}$ admits a traceless lift of the inversion provided the instanton number is even, say $2 k$. As before, we only have to show that the function $\Phi(x)=g(-x)^{-1} \Phi g(x)$ on $S^{3}=\{|x|=\epsilon\}$ extends to $|x| \leq \epsilon$ as a function obeying $\Phi(-x) \Phi(x)=1$ and $\Phi(x)^{\dagger} \Phi(x)=1$, if $g: S^{3} \rightarrow U(2 N)$ is a map of even winding number $2 k$. We choose $g(x)$ to be $\operatorname{diag}\left(g_{2}(x), \mathbf{1}_{N-2}, g_{2}(x), \mathbf{1}_{N-2}\right)$ where $g_{2}(x)$ is a map $S^{3} \rightarrow S U(2)$ of winding number $k$ such that $g_{2}(-x)=(-1)^{k} g_{2}(x)$. (it is easy to construct such $g_{2}(x)$ : for example, identify $x \in S^{3}$ as a unit quaternion and put $\left.g_{2}(x)=x^{k} \in S p(1)=S U(2)\right)$. Then, $\Phi(x)$ is a constant unitary matrix $\Phi^{\prime}$ such that $\Phi^{\prime 2}=\mathbf{1}_{2 N}$ and $\operatorname{Tr} \Phi^{\prime}=0$, and therefore extends to $|x| \leq \epsilon$.

\subsection{Nahm Transform as T-Duality}

We now describe T-duality in terms of the gauge field configuration representing the system. We consider T-duality transform of $\mathrm{D}(p+4)$ and $\mathrm{D} p$-branes on $T^{4}$ where $T^{4}$ is (i) at the fixed plane of orthogonal-orientifold, (ii) at the fixed plane of symplectic-orientifold, and (iii) divided by a $\mathbf{Z}_{2}$ orbifold action.

\section{(i) Orthogonal Bundle $\leftrightarrow$ Symplectic Orientibundle}

We first consider an (unphysical) Type I string theory (or equivalently Type IIB orientifold of $S O$-type) on $\mathbf{R}^{6} \times T^{4}$ with $N$ D9-branes wrapped on $T^{4}$ and $2 k$ D5-branes at points on $T^{4}$.

\footnotetext{
${ }^{5} \mathrm{~A}$ more standard terminology (after the $\mathbf{Z}_{2}$ quotient) is $V$-bundle over $V$-manifold. The name "orbibundle" is due to K. Fukaya and K. Ono (as far as I know). "Orientibundle" which I introduced in the previous part of the paper simply follows this terminology.
} 
The D9-branes support an $S O(N)$ gauge field and the D5-branes can be represented by an $S O(N)$ instanton on $T^{4}$. An $S O(N)$ gauge field can be considered as a connection of a $U(N)$ bundle $E$ preserving an orthogonal structure $I$ of $E$ (i.e. anti-linear isometric involutions $I_{x}: E_{x} \rightarrow E_{x}$ ). Note that the embedding $S O(N) \hookrightarrow U(N)$ has index 2, namely, it maps the generator of $\pi_{3}(S O(N)) \cong \mathbf{Z}$ to two times the generator of $\pi_{3}(U(N)) \cong \mathbf{Z}$. Therefore the $2 k$ D5-branes, which are represented by a $2 k$-instanton of $U(N)$ group, correspond to a $k$-instanton of $S O(N)$.

\section{Probing by a Wrapped D5-brane Pair}

We probe this system by a pair of D5-branes wrapped on $T^{4}$ and spanning the $x^{ \pm}$directions. The theory on the probe is a $(1,0)$ supersymmetric $S U(2)$ gauge theory in $5+1$ dimensions with $S O(N)$ flavor symmetry where the eight supersymmetries are broken to half by the instanton configuration of the flavor group. The theory has an $S U(2)$ vector multiplet, a singlet hypermultiplet and a half-hypermultiplet in the bifundamental representation $\left(\mathbf{N}, \mathbf{2}^{*}\right)$ of $S O(N) \times S U(2)$. The condition of half-hypermultiplet is important for our purpose and deserves a paragraph of digression as a reminder.

If a hypermultiplet is in a pseudo-real representation of the flavor $\times$ gauge group, one can impose a half-hypermultiplet condition. Let $J^{A B}$ be the skew-form defining the psudo-reality (so that the representation matrix $g_{B}^{A}$ satisfies $J^{A B} \overline{g_{C}^{B}}=g_{B}^{A} J^{B C}$ in addition to unitarity), and let $\epsilon^{\sigma \tau}$ be the invariant tensor of the $S U(2)_{R}$ symmetry. Then, the half-hypermultiplet conditions for the hypermultiplet fields $\left(Q^{\sigma A}, \Psi^{A}\right)$ are

$$
\begin{aligned}
& Q^{\sigma A}=J^{A B} \epsilon^{\tau \sigma} \overline{Q^{\tau B}}, \\
& \Psi^{A}=J^{A B}\left(\Psi^{B}\right)^{c_{5+1}},
\end{aligned}
$$

where $(\cdot)^{c_{5+1}}$ is the charge conjugation in $5+1$ dimensions. ${ }^{1}$ In particular,

\footnotetext{
${ }^{1}$ In $4 n$ dimensional Euclidean or $((4 n+1)+1)$ dimensional Minkowski space, charge conjugation does not flip the chirality. The square of charge conjugation is 1 for even $n$ while it is -1 for odd $n$. The $5+1$ dimensional spinor representation decomposes to the tensor product of the 4 and $1+1$ dimensional spinor representations, and the $5+1$ dimensional charge conjugation can be represented as the tensor product of the ones in 4 and $1+1$ dimensions;
}

$$
(\psi \otimes \lambda)^{c_{5+1}}=\psi^{c_{4}} \otimes \lambda^{c_{1+1}} .
$$

We shall write $(\cdot)^{c}$ for $(\cdot)^{c_{4}}$. In the representation where the $d=4$ Gamma matrices 
the fermion $\Psi^{a}$ is a symplectic-Majorana-Weyl spinor (of negative chirality). Indeed, the conditions (3.16) and (3.17) are invariant under the $(1,0)$ supersymmetry

$$
\delta Q^{\sigma A}=\overline{\xi_{\sigma}} \Psi^{A}, \quad \delta \Psi^{A}=\left(\not D Q^{\sigma}\right)^{A} \xi_{\sigma}
$$

generated by a symplectic-Majorana-Weyl spinor $\xi_{\sigma}=\epsilon_{\sigma \tau}\left(\xi_{\tau}\right)^{c_{5+1}}$ of positive chirality. In our case, the flavor $\times$ gauge group is $S O(N) \times S U(2)$ and the representation $\left(\mathbf{N}, \mathbf{2}^{*}\right)$ is indeed pseudo-real; if we denote the $S U(2)$-gauge indices by a, b, $\ldots$ and the $S O(N)$-flavor indices by $i, j, \ldots$, the skew-form defining the pseudo-reality is $\epsilon^{\mathrm{ab}} \delta^{i j}$. In the present set-up, the $S O(N)$ flavor bundle is topologically non-trivial and the hypermultiplet fields are sections $Q^{\sigma \mathrm{a}}$ and $\Psi^{\mathrm{a}}$ of the bundle $E$ and $E \otimes S_{5+1}^{-}$respectively where $S_{5+1}^{-}$ is the $5+1$ dimensional spin bundle of negative chirality. Let us define an antilinear map $I^{c_{5+1}}: E \otimes S_{5+1}^{-} \rightarrow E \otimes S_{5+1}^{-}$as the tensor product of the map $I$ acting on $E$ and the charge conjugation on $S_{5+1}^{-}$. Then, the conditions of half-hypermultiplet are $Q^{\sigma \mathrm{a}}=\epsilon^{\mathrm{ab}} \epsilon^{\tau \sigma} I\left(Q^{\tau \mathrm{b}}\right)$ and

$$
\Psi^{\mathrm{a}}=\epsilon^{\mathrm{ab}} I^{c_{5+1}}\left(\Psi^{\mathrm{b}}\right)
$$

where $I Q$ is defined by $(I Q)(x)=I_{x} Q(x)$ and the definition of $I^{c_{5+1}} \Psi$ is similar.

At long distances, as in the case without orientifold projection, we obtain an effective $1+1$ dimensional theory with $(0,4)$ supersymmetry. The $S U(2)$ gauge field on $T^{4}$ reduces to scalar fields taking values in the moduli space of flat $S U(2)$ connections on $T^{4}$. A flat $S U(2)$ connection can be represented as a constant gauge field of the form

$$
a_{\mu}^{S U(2)}=i\left(\begin{array}{cc}
a_{\mu} & 0 \\
0 & -a_{\mu}
\end{array}\right) .
$$

Since there are $S U(2)$ gauge equivalence relations $a_{\mu} \equiv-a_{\mu} \equiv a_{\mu}+\hat{n}_{\mu}$ $\left(\hat{n} \in 2 \pi \Lambda^{*}\right)$, the moduli space is $\left(\mathbf{R}^{4}\right)^{*} /\left(2 \pi \Lambda^{*} \tilde{\times} \mathbf{Z}_{2}\right)=\widehat{T}^{4} / \mathbf{Z}_{2}$. Away from the $\mathbf{Z}_{2}$ fixed points of $\widehat{T}^{4}$, the $S U(2)$ gauge symmetry is broken to $U(1)$ and

are given by

$$
\gamma^{\mu}=\left(\begin{array}{cc}
0 & \sigma^{\mu} \\
\bar{\sigma}^{\mu} & 0
\end{array}\right), \quad \sigma^{\mu}=(i \vec{\sigma}, 1), \quad \bar{\sigma}^{\mu}=\sigma^{\mu \dagger},
$$

the $d=4$ charge conjugation is given by $\psi^{c}=C \psi^{*}$ (on both chirality) in which $C$ is a charge conjugation matrix given by $C=i \sigma_{2}$ and $\psi^{*}$ is the complex conjugation of $\psi$.

The $d=1+1$ charge conjugation is simply the complex conjugation $\lambda^{c_{1+1}}=\lambda^{*}$ in the representation where $\gamma^{0}=-i \sigma_{2}$ and $\gamma^{9}=\sigma_{1}$ in which $\gamma^{0} \gamma^{9}=\operatorname{diag}(-1,1)$. In this paper, we often denote $\lambda^{*}$ by $\bar{\lambda}$. 
we obtain from the $S U(2)$ vector multiplet and the singlet hypermultiplet the $1+1$ dimensional $(0,4)$ multiplets whose bosonic components are $U(1)$ gauge field $i a_{ \pm}$(embedded in $S U(2)$ as $a_{ \pm}^{S U(2)}=\operatorname{diag}\left(i a_{ \pm},-i a_{ \pm}\right)$) and scalar fields taking values in $\mathbf{R}^{4} \times \widehat{T}^{4}$ modulo a $\mathbf{Z}_{2}$ action which simultaneously flips the sign of $a_{ \pm}$and the coordinates of $\widehat{T}^{4}$. At the sixteen $\mathbf{Z}_{2}$ fixed points, the $S U(2)$ gauge symmetry is restored.

Massless fields also come from the half-hypermultiplet in the $\left(\mathbf{N}, \mathbf{2}^{*}\right)$ representation. They correspond to the fields on $T^{4}$ satisfying the equations as (2.7) where now

$$
D_{\mu}=\partial_{\mu}+A_{\mu}^{S O(N)}-{ }^{t} a_{\mu}^{S U(2)},
$$

in which $a_{\mu}^{S U(2)}$ is the flat $S U(2)$ gauge field (3.20) and $A_{\mu}^{S O(N)}$ is the $S O(N)$ instanton. Namely, they come from the zero modes of the Laplace and the Dirac operators on $T^{4}$ associated with $D_{\mu}^{( \pm a)}=\partial_{\mu}+A_{\mu}^{S O(N)} \mp i a_{\mu}$. Generically, there are nothing else than $2 k$ negative-chirality zero modes for each of $\not^{(a)}$ and $\not D^{(-a)}$. As in the previous section, we expand the field $\Psi^{\mathrm{a}}$ by the orthonormal basis $\psi_{I}( \pm a)(I=1, \ldots, 2 k)$ of the space of zero modes of $\not D^{( \pm a)}$ as

$$
\Psi^{1}=\sum_{I=1}^{2 k} \psi_{I}(a) \otimes \lambda^{1 I}, \quad \Psi^{2}=\sum_{I=1}^{2 k} \psi_{I}(-a) \otimes \lambda^{2 I},
$$

where $\lambda^{\mathrm{a}}=\left(\lambda^{\mathrm{a} I}\right)$ are positive chirality spinors in $1+1$ dimensions. The lagrangian for $\Psi^{a}$ then becomes

$$
\left(\overline{\lambda^{1}}, \overline{\lambda^{2}}\right)\left(\partial_{-}+\partial_{-} a_{\mu}\left(\begin{array}{cc}
\hat{A}^{\mu}(a) & 0 \\
0 & -\hat{A}^{\mu}(-a)
\end{array}\right)-{ }^{t} a_{-}^{S U(2)}\right)\left(\begin{array}{l}
\lambda^{1} \\
\lambda^{2}
\end{array}\right)
$$

where $\hat{A}^{\mu}(a) \mathrm{d} a_{\mu}$ is the $U(2 k)$ gauge field on $\widehat{T}^{4}$ (defined as in (2.10)) defining a connection of the bundle $\hat{E}$ of $\not^{(a)}$ zero modes. The bundle $\hat{E}$ has instanton number $N$ and $\hat{A}=\hat{A}^{\mu}(a) \mathrm{d} a_{\mu}$ has a self-dual curvature.

The half-hypermultiplet condition constrains the fermions $\lambda^{\mathrm{a}}$. We first note that the operator $I^{c_{5+1}}$ induces in the $T^{4}$ factor a map sending $\not^{(a)}$ zero modes to $\not D^{(-a)}$ zero modes and vice versa. Let $I^{c}: E \otimes S \rightarrow E \otimes S$ be the tensor product map of $I$ on $E$ and the charge conjugation on the spinor bundle $S$ on $T^{4}$. With respect to a (local) real-orthonormal frame of $E, I^{c}$ is represented simply as the charge conjugation. Since $A_{\mu}^{S O(N)}$ is represented by real anti-symmetric matrices in such a frame, the effect of $I^{c}$ on the Dirac 
equation $\gamma^{\mu}\left(\partial_{\mu}+A_{\mu}^{S O(N)}-i a_{\mu}\right) \psi=0$ is simply to change the sign of $a_{\mu}$. Namely, if $\psi$ is a zero mode of $\not D^{(a)}$, then $I^{c} \psi$ (defined as $\left(I^{c} \psi\right)(x)=I_{x}^{c} \psi(x)$ ) is a zero mode of $\not^{(-a)}$. Thus, $I^{c} \psi_{I}(a)$ can be spanned by $\psi_{I}(-a)$ 's

$$
I^{c} \psi_{I}(a)=\sum_{J} \psi_{J}(-a) J_{a}^{J I}
$$

Then, the half-hypermultiplet condition $\Psi^{2}=I^{c_{5+1}} \Psi^{1}$ requires that

$$
\lambda^{2 I}=J_{a}^{I J} \overline{\lambda^{1 J}} .
$$

The anti-linear map $J_{a}: \hat{E}_{a} \rightarrow \hat{E}_{-a}$ induced by $\psi \mapsto I^{c} \psi$ defines a symplectic structure $J$ over the inversion. Indeed, it squares to $J_{-a} J_{a}=-1$ due to the property $\psi^{c c}=-\psi$ of charge conjugation in Euclidean four dimension, and it is isometric since $\left(I^{c} \psi_{1}\right)^{\dagger} I^{c} \psi_{2}=\psi_{2}^{\dagger} \psi_{1}$. A direct computation shows

$$
J_{a}^{I J} \overline{\hat{A}^{\mu}(a)_{\bar{J} K}}=-\hat{A}^{\mu}(-a)_{\bar{I} J} J_{a}^{J K},
$$

which means that the connection $\hat{A}$ preserves $J$. Thus, we have a symplectic orientibundle $(\hat{E}, J)$ over the orientifold $\widehat{T}^{4} / \mathbf{Z}_{2}$ with a connection $\hat{A}$.

The theory we have obtained is exactly the same as the effective theory on a D1-brane pair probing the system of $k$ pairs of D9-branes in Type IIB orientifold on $\mathbf{R}^{6} \times \widehat{T}^{4} / \mathbf{Z}_{2}$ which support the symplectic orientibundle $(\hat{E}, J)$ with the connection $\hat{A}$. In fact, the condition (3.25) is nothing but the orientifold projection on 1-9 open string modes. By definition, this theory must be identifed as the effective theory of the D1-brane pair which is Tdual to the original D5-brane pair. Thus, we conclude that the T-duality mapping D9 and D5 branes in Type I string theory on $\mathbf{R}^{6} \times T^{4}$ to D9 and D5 branes in Type IIB orientifold on $\mathbf{R}^{6} \times \widehat{T}^{4} / \mathbf{Z}_{2}$ is represented by the transform $\left(E, A^{S O(N)}, I\right) \mapsto(\hat{E}, \hat{A}, J)$ of $S O(N)$ instantons on $T^{4}$ of instanton number $k$ to $N$-instantons in symplectic orientibundle on $\widehat{T}^{4} / \mathbf{Z}_{2}$ of rank $2 k$.

\section{The Inverse Transform}

The transform $\left(E, A^{S O(N)}, I\right) \mapsto(\hat{E}, \hat{A}, J)$ may be considered as a generalization of Nahm transform to the case of orthogonal bundles. To fully establish this, we should provide the inverse transform. Thus, we consider D9-branes wrapped on the orthogonal orientifold $\widehat{T}^{4} / \mathbf{Z}_{2}$ supporting a rank 
$2 k$ symplectic orientibundle $(E, A, J)$ of instanton number $N$, and probe this by a wrapped D5-brane.

The theory of lowlying modes on the probe D5-brane can be analyzed in the same way as we have done for wrapped D4-branes in orientifold on $\mathbf{R} \times\left(\widehat{T}^{4} \times \mathbf{R}^{5}\right) / \mathbf{Z}_{2}$. It is a $5+1$ dimensional $(1,0)$ supersymmetric $U(1)$ gauge theory on $\mathbf{R}^{2} \times \widehat{T}^{4}$ with $U(2 k)$ flavor symmetry where the eight supersymmetries are broken to half by constraints on the fields (written below) and by the instanton configuration $A^{\mu} \mathrm{d} \hat{x}_{\mu}$ of the flavor group. The theory containes a $U(1)$ vector multiplet, a free hypermultiplet and a hypermultiplet in the bifundamental representation $(\mathbf{2 k},-1)$ of $U(2 k) \times U(1)$. The bosonic fields $X^{p}$ from the free hypermultiplet and the $U(1)$ gauge field $i a$ (and their superpartners) are subject to the constraints

$$
\begin{aligned}
& X^{p}(-\hat{x})=X^{p}(\hat{x}), \\
& a^{\mu}(-\hat{x})=a^{\mu}(\hat{x}), a_{ \pm}(-\hat{x})=-a_{ \pm}(\hat{x}),
\end{aligned}
$$

and the $U(1)$ gauge transformations are also constrained as $g(-\hat{x})=g(\hat{x})^{-1}$. The hypermultiplet in the bifundamental $(\mathbf{2 k},-1)$, which consists of sections $Q^{\sigma}$ and $\Psi$ of $E$ and $E \otimes \hat{S}_{5+1}^{-}$, is subject to the "half-hypermultiplet" conditions: $Q^{\sigma}=\epsilon^{\tau \sigma} J Q^{\tau}$ and

$$
\Psi=J^{c_{5+1}} \Psi
$$

where $J Q$ and $J^{c_{5+1}} \Psi$ are defined respectively by $(J Q)(\hat{x})=J_{-\hat{x}} Q(-\hat{x})$ and $\left(J^{c_{5+1}} \Psi\right)(\hat{x})=\Gamma^{1234} J_{-\hat{x}}^{c_{5+1}} \Psi(-\hat{x})$ (multiplication by $\Gamma^{1234}$ represents a lift of the inversion of $\widehat{T}^{4}$ to the spin bundle). These conditions are invariant under the supersymmetry (3.18) generated by the symplectic-Majorana-Weyl spinor $\xi_{\sigma}$ which is of positive-chirality also in four dimensions $\Gamma^{1234} \xi_{\sigma}=\xi_{\sigma}$.

The Kaluza-Klein reduction on $\widehat{T}^{4}$ leads to a $1+1$ dimensional $(0,4)$ supersymmetric theory. The gauge symmetry reduces from $U(1)$ to $\mathbf{Z}_{2}$ since $g= \pm 1$ are the only gauge transformations that are constant along $\widehat{T}^{4}$ satisfying the constraint. The vector and the free hypermultiplets reduce to $\mathbf{Z}_{2}$-singlet $(0,4)$ multiplets whose bosonic components are scalar fields taking values in $\mathbf{R}^{4} \times T^{4}$ where $T^{4}$ appears here as the moduli space of (constrained) flat $U(1)$ connections $i x^{\mu} \mathrm{d} \hat{x}_{\mu}$ on $\widehat{T}^{4}$. The hypermultiplet in $(\mathbf{2} \mathbf{k},-1)$ leads to $\mathbf{Z}_{2}$-nonsinglet positive-chirality fermions $\lambda^{i}$ with values in the bundle $\check{E}$ of zero modes of the Dirac operator associated with the covariant derivative $D^{\mu}=\partial^{\mu}+A^{\mu}-i x^{\mu}$. The bundle $\check{E}$ has rank $N$ and instanton number $2 k$. The fermions $\lambda^{i}$ are coupled to the dual connection $\check{A}_{\mu}(x)_{\bar{i} j}$ of $\check{E}$ constructed 
as before, and are constrained as below.

Let $J^{c}: E \otimes \hat{S} \rightarrow E \otimes \hat{S}$ be the anti-linear map covering the inversion of $\widehat{T}^{4}$ defined as the tensor product of the map $J: E \rightarrow E$ and the charge conjugation followed by the $\gamma^{1234}$-multiplication on the spinor bundle $\hat{S}$ of $\widehat{T}^{4}$ (the latter map is an antilinear lift of the inversion of $\widehat{T}^{4}$ to $\hat{S}$ ). In a (local) symplectic frame of $E$, it is represented as the charge conjugation followed by the multiplication by $\gamma^{1234}$ and the matrix $J$ given in (3.1). Then, the Dirac equation $\gamma_{\mu}\left(\partial^{\mu}+A^{\mu}(\hat{x})-i x^{\mu}\right) \psi(\hat{x})=0$ is transformed to $\gamma_{\mu}\left(\partial^{\mu}+J \overline{A^{\mu}(\hat{x})} J^{-1}+i x^{\mu}\right) \gamma^{1234} J \psi^{c}(\hat{x})=0$. In the symplectic frame, the gauge field $A^{\mu}(\hat{x})$ satisfies (3.5); $J \overline{A^{\mu}(\hat{x})} J^{-1}=-A^{\mu}(-\hat{x})$. Thus, we see that $J^{c} \psi$ (defined as $\left.\left(J^{c} \psi\right)(\hat{x})=J_{-\hat{x}}^{c} \psi(-\hat{x})\right)$ is a $\not D$ zero mode, if $\psi$ is. If $\psi_{i}$ $(i=1, \ldots, N)$ are orthonormal basis of the space of $\not D$ zero modes, $J^{c} \psi_{i}$ can be spanned by $\psi_{j}$ 's

$$
J^{c} \psi_{i}=\psi_{j} \check{I}_{x}^{j i}
$$

Then, the condition (3.29) constrains the fermions $\lambda^{i}$ (associated with the base $\left.\psi_{i}\right)$ as

$$
\lambda^{i}=\check{I}_{x}^{i j} \overline{\lambda^{j}}
$$

The anti-linear map $\check{I}_{x}: \check{E}_{x} \rightarrow \check{E}_{x}$ induced by $\psi \mapsto J^{c} \psi$ defines an orthogonal structure of $\check{E}$. Indeed, it is an involution $\check{I}^{2}=1$ because $\psi^{c c}=$ $-\psi$ and $J J=-1$, and also it is an isometry, $\int J^{c} \psi_{1}^{\dagger} J^{c} \psi_{2}=\int \psi_{2}^{\dagger} \psi_{1}$. It is easy to see that the dual gauge field $\check{A}$ preserves this. Thus, we have an $S O(N)$ bundle $(\check{E}, \check{I})$ with an $S O(N)$ connection $\check{A}$.

The effective theory we have obtained is exactly the same as the effective theory of a D1-brane probing the system of $N$ D9-branes in Type I string theory on $\mathbf{R}^{6} \times T^{4}$ which support the $S O(N)$ bundle $(\check{E}, \check{I})$ and the connection $\check{A}$. Indeed, a Type I D1-brane has a $\mathbf{Z}_{2}$ gauge symmetry [24] and also, (3.31) is nothing but the orientifold projection on 1-9 string modes in such a system. Thus, we can conclude that the T-duality mapping the D9D5 system in Type IIB orientifold on $\mathbf{R}^{6} \times \widehat{T}^{4} / \mathbf{Z}_{2}$ to the D9-D5 system in Type I on $\mathbf{R}^{6} \times T^{4}$ is represented by the transform $(E, A, J) \mapsto(\check{E}, \check{A}, \check{I})$ of the gauge field configurations. Since T-duality squares to the identity, the transforms $(E, A, I) \rightarrow(\hat{E}, \hat{A}, J)$ and $(E, A, J) \mapsto(\check{E}, \check{A}, \check{I})$ must be inverse of each other. This can indeed be shown explicitly (see Appendix). 


\section{Summary}

We have constructed a Nahm transform which is a correspondence of instantons of orthogonal bundle on $T^{4}$ and instantons of symplectic orientibundle on the orthogonal orientifold $\widehat{T}^{4} / \mathbf{Z}_{2}$. The orthogonal bundle of rank $N$ and instanton number $k$ correspond to the symplectic orientibundle of rank $2 k$ and instanton number $N$. By construction, the T-duality between the D9D5 system of Type I string theory on $T^{4}$ and the D9-D5 system of Type IIB on orthogonal orientifold $\widehat{T}^{4} / \mathbf{Z}_{2}$ is given by this Nahm transform.

\section{(ii) Symplectic Bundle $\leftrightarrow$ Orthogonal Orientibundle}

We next consider an (unphysical) Type IIB string theory with $S p$-type O9plane on $\mathbf{R}^{6} \times T^{4}$ with $2 N$ D9-branes wrapped on $T^{4}$ and $k$ D5-branes at points on $T^{4}$.

The D9-branes support an $S p(N)$ gauge field which can be considered as a connection of a $U(2 N)$ bundle $E$ preserving a symplectic structure $J$ of $E$ (i.e. anti-linear isometries $J_{x}: E_{x} \rightarrow E_{x}$ such that $J_{x}^{2}=-\mathrm{id}_{x}$ ). Since the embedding $S p(N) \hookrightarrow U(2 N)$ has index one, the $k$ D5-branes correspond to a $k$-instanton of $S p(N)$ on $T^{4}$.

\section{Probing by a Wrapped D5-brane Pair}

We probe the system by a pair of wrapped D5-branes as before. The theory on the probe is a $(1,0)$ supersymmetric $O(2)$ gauge theory in $5+1$ dimensions with $S p(N)$ flavor symmetry where the supersymmetries are broken to half by the instanton configuration of the flavor group. The theory has an $O(2)$ vector multiplet, a hypermultiplet in the second rank symmetric tensor representation, and a half-hypermultiplet in the bifundamental representation $\left(\mathbf{2 N}, \mathbf{2}^{*}\right)$ of $S p(N) \times O(2)$. The conditions of half-hypermultiplet are $Q^{\sigma \mathrm{a}}=\epsilon^{\tau \sigma} J Q^{\tau \mathrm{a}}$ and $\Psi^{\mathrm{a}}=J^{c_{5+1}} \Psi^{\mathrm{a}}$ where $\mathrm{a}, \mathrm{b}=1,2$ are $O(2)$ gauge indices, $Q^{\sigma \mathrm{a}}$ and $\Psi^{\mathrm{a}}$ are the hypermultiplet fields which are sections of the bundle $E$ and $E \otimes S_{5+1}^{-}$respectively, and $J Q$ and $J^{c_{5+1}} \Psi$ are defined as before.

At long distances, we obtain an effective $1+1$ dimensional theory with $(0,4)$ supersymmetry. The $O(2)$ gauge field on $T^{4}$ reduces to scalar fields 
taking values in the moduli space of flat $O(2)$ connections on $T^{4}$. A flat $O(2)$ connection can be represented with respect to a complexified $O(2)$ base (such that the fermion $\Psi$ is represented as $\Psi^{ \pm}=\Psi^{1} \mp i \Psi^{2}$ in the corresponding dual basis) as a constant field

$$
a_{\mu}^{O(2)}=i\left(\begin{array}{cc}
a_{\mu} & 0 \\
0 & -a_{\mu}
\end{array}\right) .
$$

Since there are $O(2)$ gauge equivalence relations $a_{\mu} \equiv-a_{\mu} \equiv a_{\mu}+\hat{n}_{\mu}(\hat{n} \in$ $\left.2 \pi \Lambda^{*}\right)$, the moduli space is $\widehat{T}^{4} / \mathbf{Z}_{2}$. Away from the $\mathbf{Z}_{2}$ fixed point, the gauge symmetry is broken to $S O(2)=U(1)$ and the $O(2)$ vector multiplet plus the symmetric tensor hypermultiplet reduce to a $U(1)$ gauge field $a_{ \pm}^{O(2)}$ and scalar fields taking values in $\mathbf{R}^{4} \times \widehat{T}^{4}$ (with their superpartners) modulo a $\mathbf{Z}_{2}$ action which flips the sign of $a_{ \pm}^{O(2)}$ and the coordinates of $\widehat{T}^{4}$ (and their superpartners). At the sixteen fixed points, the $O(2)$ gauge symmetry is restored and a new branch $\mathbf{R}^{4} / \mathbf{Z}_{2}$ develops.

Massless fields also come from the half-hypermultiplet in the $\left(\mathbf{2 N}, \mathbf{2}^{*}\right)$ representation. They correspond to the fields on $T^{4}$ obeying the equations as (2.7) where now

$$
D_{\mu}=\partial_{\mu}+A_{\mu}^{S p(N)}-a_{\mu}^{O(2)},
$$

in which $a_{\mu}^{O(2)}$ is the flat gauge field (3.32) and $A_{\mu}^{S p(N)}$ is the $S p(N)$ instanton. Namely, they come from the zero modes of the Laplace and the Dirac operators associated with $D_{\mu}^{( \pm a)}=\partial_{\mu}+A_{\mu}^{S p(N)} \mp i a_{\mu}$. Generically there are nothing else than $k$ Dirac zero modes of negative-chirality. We expand $\Psi^{ \pm}=\Psi^{1} \mp i \Psi^{2}$ by the orthonormal basis $\psi_{I}( \pm a)(I=1, \ldots, k)$ of the space of $\not D^{( \pm a)}$ zero modes as $\Psi^{ \pm}=\sum \psi_{I}( \pm a) \otimes \lambda^{ \pm I}$ where $\lambda^{ \pm}=\left(\lambda^{ \pm I}\right)$ are $1+1$ dimensional positive-chirality fermions. Then, the lagrangian for $\Psi^{ \pm}$becomes

$$
\left(\overline{\lambda^{+}}, \overline{\lambda^{-}}\right)\left(\partial_{-}+\partial_{-} a_{\mu}\left(\begin{array}{cc}
\hat{A}^{\mu}(a) & 0 \\
0 & -\hat{A}^{\mu}(-a)
\end{array}\right)-a_{-}^{O(2)}\right)\left(\begin{array}{c}
\lambda^{+} \\
\lambda^{-}
\end{array}\right)
$$

where $\hat{A}^{\mu}(a) \mathrm{d} a_{\mu}$ is the $U(k)$ connection of the bundle $\hat{E}$ over $\widehat{T}^{4}$ of $\not^{(a)}$ zero modes defined as before. The bundle $\hat{E}$ has instanton number $2 N$ and $\hat{A}=\hat{A}^{\mu}(a) \mathrm{d} a_{\mu}$ has a self-dual curvature.

The half-hypermultiplet condition $\Psi^{ \pm}=J^{c_{5+1}} \Psi^{\mp}$ constrains $\lambda^{ \pm}$. Let $J^{c}: E \otimes S \rightarrow E \otimes S$ be the tensor product map of $J: E \rightarrow E$ and the charge conjugation on the spinor bundle $S$ on $T^{4}$. With respect to a (local) 
symplectic frame of $E, J^{c}$ is represented by the charge conjugation followed by the multiplication by the $2 N \times 2 N$ matrix $J$ as (3.1) under which the Dirac equation $\gamma^{\mu}\left(\partial_{\mu}+A_{\mu}^{S p(N)}-i a_{\mu}\right) \psi=0$ is transformed to $\gamma^{\mu}\left(\partial_{\mu}+J \overline{A_{\mu}^{S p(N)}} J^{-1}+\right.$ $\left.i a_{\mu}\right) J \psi^{c}=0$. In such a frame, the $S p(N)$ gauge field obeys $J \overline{A_{\mu}^{S p(N)}}=$ $A_{\mu}^{S p(N)} J$. Thus, if $\psi$ is a zero mode of $\not^{(a)}$, then $J^{c} \psi$ (defined as $\left(J^{c} \psi\right)(x)=$ $\left.J_{x}^{c} \psi(x)\right)$ is a zero mode of $\not^{(-a)}$. In particular, $J^{c} \psi_{I}(a)$ is spanned by $\psi_{I}(-a)$ 's

$$
J^{c} \psi_{I}(a)=\sum_{J} \psi_{J}(-a) I_{a}^{J I}
$$

Thus, the half-hypermultiplet condition requires

$$
\lambda^{-I}=I_{a}^{I J} \overline{\lambda^{+J}} .
$$

The anti-linear map $I_{a}: \hat{E}_{a} \rightarrow \hat{E}_{-a}$ induced by $\psi \mapsto J^{c} \psi$ defines an orthogonal structure over the inversion of $\widehat{T}^{4}$. Indeed, it squares to $I_{-a} I_{a}=1$ because $\psi^{c c}=-\psi$ and $J J=-1$, and is isometric since $\left(J^{c} \psi_{1}\right)^{\dagger} J^{c} \psi_{2}=$ $\psi_{2}^{\dagger} \psi_{1}$. It is easy to see that the connection $\hat{A}$ preserves $I$. Thus, we have an orthogonal orientibundle $(\hat{E}, I)$ with a connection $\hat{A}$.

The theory we have obtained is the same as the effective theory on a D1-brane pair probing the system of $k$ D9-branes in Type IIB symplecticorientifold on $\mathbf{R}^{6} \times \widehat{T}^{4} / \mathbf{Z}_{2}$ which support the orthogonal orientibundle $(\hat{E}, I)$ with the connection $\hat{A}$. In fact, the condition (3.36) is nothing but the orientifold projection on 1-9 string modes. Thus, we conclude that the Tduality mapping the D9-D5 system in Type IIB symplectic-orientifold on $\mathbf{R}^{6} \times T^{4}$ to the D9-D5 system in Type IIB symplectic-orientifold on $\mathbf{R}^{6} \times$ $\widehat{T}^{4} / \mathbf{Z}_{2}$ is represented by the transform $\left(E, A^{S p(N)}, J\right) \mapsto(\hat{E}, \hat{A}, I)$ of $S p(N)$ instantons on $T^{4}$ of instanton number $k$ to $2 N$-instantons in orthogonal orientibundle on $\widehat{T}^{4} / \mathbf{Z}_{2}$ of rank $k$.

\section{The Inverse Transform}

To find the inverse transform, we consider D9-branes wrapped on the symplectic orientifold $\widehat{T}^{4} / \mathbf{Z}_{2}$ supporting an orthogonal orientibundle $(E, A, I)$ of rank $k$ and instanton number $2 N$, and probe it by a wrapped D5-brane pair.

The theory on the probe is a $5+1$ dimensional $(1,0)$ supersymmetric $U(2)$ gauge theory on $\mathbf{R}^{2} \times \widehat{T}^{4}$ with $U(k)$ flavor symmetry where the eight 
supersymmetries are broken to half by constraints on the fields (written below) and by the instanton configuration $A^{\mu} \mathrm{d} \hat{x}_{\mu}$ of the flavor group. The theory containes a $U(2)$ vector multiplet, an adjoint hypermultiplet and a hypermultiplet in the bifundamental representation $\left(\mathbf{k}, \mathbf{2}^{*}\right)$ of $U(k) \times U(2)$. The bosonic fields $X^{p}$ from the adjoint hypermultiplet and the $U(2)$ gauge field $a_{U(2)}$ (and their superpartners) are subject to the constraints

$$
\begin{aligned}
& X^{p}(-\hat{x})=\epsilon X^{p}(\hat{x})^{T} \epsilon^{-1}, \\
& a_{U(2)}^{\mu}(-\hat{x})=\epsilon a_{U(2)}^{\mu}(\hat{x})^{T} \epsilon^{-1}, \quad a_{ \pm}^{U(2)}(-\hat{x})=-\epsilon a_{ \pm}^{U(2)}(\hat{x})^{T} \epsilon^{-1},
\end{aligned}
$$

where $\epsilon$ is the $2 \times 2$ matrix as $J$ in (3.1). The gauge transformations are also constrained as $\epsilon \overline{g(\hat{x})}=g(-\hat{x}) \epsilon$. The hypermultiplet in $\left(\mathbf{k}, \mathbf{2}^{*}\right)$ consisting of sections $Q^{\sigma \mathrm{a}}$ and $\Psi^{\mathrm{a}}$ of $E$ and $E \otimes \hat{S}_{5+1}^{-}(\mathrm{a}=1,2$ are $U(2)$ gauge indices) is subject to the "half-hypermultiplet" conditions: $Q^{\sigma \mathrm{a}}=\epsilon^{\mathrm{ab}} \epsilon^{\tau \sigma} I Q^{\tau \mathrm{b}}$ and

$$
\Psi^{\mathrm{a}}=\epsilon^{\mathrm{ab}} I^{c_{5+1}} \Psi^{\mathrm{b}}
$$

where $I Q$ and $I^{c_{5+1}} \Psi$ are defined by $(I Q)(\hat{x})=I_{-\hat{x}} Q(-\hat{x})$ and $I^{c_{5+1}} \Psi(\hat{x})=$ $\Gamma^{1234} I_{-\hat{x}}^{c_{5+1}} \Psi(-\hat{x})$. These conditions are invariant under the supersymmetry (3.18) generated by the symplectic-Majorana-Weyl spinor $\xi_{\sigma}$ which is positive also in four dimensions $\Gamma^{1234} \xi_{\sigma}=\xi_{\sigma}$.

The Kaluza-Klein reduction on $\widehat{T}^{4}$ leads to a $1+1$ dimensional $(0,4)$ supersymmetric theory. The $U(2)$ gauge symmetry reduces to $S U(2)$, since gauge transformations and the gauge field $a_{ \pm}^{U(2)}$ that are constant on $\widehat{T}^{4}$ belong to the $S U(2)$ subgroup. The $\widehat{T}^{4}$ components of the gauge field reduces to the scalar fields with values in the moduli space of flat $U(2)$ connection on $\widehat{T}^{4}$ subject to the constraint (3.38). Such a flat connection can be expressed as $a_{U(2)}^{\mu}=i x^{\mu} \mathbf{1}_{2}$ where $x^{\mu}$ are parameters, and the moduli space is $T^{4}$ because there are gauge equivalence relations $x^{\mu} \equiv x^{\mu}+n^{\mu}(n \in \Lambda)$. The form of $a_{U(2)}^{\mu}$ shows that these scalar fields are $S U(2)$ singlets. The adjoint hypermultiplet subject to (3.37) similarly reduces to $S U(2)$ singlet free scalar multiplet with values in $\mathbf{R}^{4}$. The bifundamental hypermultiplet reduces to $S U(2)$-doublet positive-chirality-fermions $\lambda^{a i}$ with values in the bundle $\breve{E}$ over $T^{4}$ of zero modes of the Dirac operator associated with the covariant derivative $D^{\mu}=\partial^{\mu}+A^{\mu}-i x^{\mu}$. The bundle $\check{E}$ has rank $2 N$ and instanton number $k$. The fermions $\lambda^{a i}$ are coupled to the dual connection $\check{A}_{\bar{\imath} j}$ of $\check{E}$ constructed as before, and are constrained as described below.

Let $I^{c}: E \otimes \hat{S} \rightarrow E \otimes \hat{S}$ be the anti-linear map over the inversion of $\widehat{T}^{4}$ defined as the tensor product of the map $I: E \rightarrow E$ and the charge 
conjugation followed by the $\gamma^{1234}$-multiplication on the spinor bundle $\hat{S}$. In a (local) real orthogonal frame of $E$, it is simply represented as the charge conjugation followed by the $\gamma^{1234}$-multiplication. This transforms the Dirac equation $\gamma_{\mu}\left(\partial^{\mu}+A^{\mu}(\hat{x})-i x^{\mu}\right) \psi(\hat{x})=0$ to $\gamma_{\mu}\left(\partial^{\mu}+\overline{A^{\mu}(\hat{x})}+i x^{\mu}\right) \gamma^{1234} \psi^{c}(\hat{x})=0$. In the real orthogonal frame, the gauge field $A^{\mu}(\hat{x})$ satisfies $(3.8) ; \overline{A^{\mu}(\hat{x})}=$ $-A^{\mu}(-\hat{x})$. Thus, we see that $I^{c} \psi$ (defined as $\left.\left(I^{c} \psi\right)(\hat{x})=I_{-\hat{x}}^{c} \psi(-\hat{x})\right)$ is a $\not D$ zero mode, if $\psi$ is. If $\psi_{i}(i=1, \ldots, 2 N)$ are orthonormal frame of the space of $\not D$ zero modes, $I^{c} \psi_{i}$ can be spanned by $\psi_{j}$ 's

$$
I^{c} \psi_{i}=\psi_{j} \check{J}_{x}^{j i} .
$$

Then, the condition (3.39) constrains the fermions $\lambda^{\text {ai }}$ (associated with the basis $\left.\psi_{i}\right)$ as

$$
\lambda^{\mathrm{a} i}=\epsilon^{\mathrm{ab}} \breve{J}_{x}^{i j} \overline{\lambda^{\mathrm{b} j}} .
$$

The anti-linear map $\check{J}_{x}: \check{E}_{x} \rightarrow \check{E}_{x}$ induced by $\psi \mapsto I^{c} \psi$ defines a symplectic structure on $\check{E}$. Indeed, it squares to $\check{J}^{2}=-1$ due to $\psi^{c c}=-\psi$, and is isometric since $I^{c} \psi_{1}^{\dagger} I^{c} \psi_{2}=\psi_{2}^{\dagger} \psi_{1}$. It is easy to see that the dual gauge field $\check{A}$ preserves $J$. Thus, we have an $S p(N)$ bundle $(\check{E}, \check{J})$ over $T^{4}$ with an $S p(N)$ connection $\check{A}$.

The effective theory we have obtained is the same as the effective theory of a D1-brane probing the system of D9-branes in Type II symplectic orientifold which support the $S p(N)$ bundle $(\check{E}, \check{A}, \check{J})$ on $T^{4}$. Indeed, (3.41) is nothing but the orientifold projection on 1-9 string modes. Thus, we conclude that the T-duality mapping the D9-D5 system in orientifold $\widehat{T}^{4} / \mathbf{Z}_{2}$ to the D9-D5 system in orientifold on $T^{4}$ is represented by the transform $(E, A, I) \mapsto(\check{E}, \check{A}, \breve{J})$ of the gauge field configurations. Since T-duality squares to the identity, the transforms $(E, A, J) \mapsto(\hat{E}, \hat{A}, I)$ and $(E, A, I) \mapsto(\check{E}, \check{A}, \check{J})$ must be inverse of each other. This can indeed be shown explicitly (see Appendix).

\section{Summary}

We have constructed a Nahm transform which is a correspondence of instantons of symplectic bundle on $T^{4}$ and instantons of orthogonal orientibundle on the symplectic orientifold $\widehat{T}^{4} / \mathbf{Z}_{2}$. The symplectic bundle of rank $2 N$ and instanton number $k$ correspond to the orthogonal orientibundle of rank $k$ and 
instanton number $2 N$. By construction, the T-duality between the D9-D5 system of Type IIB symplectic orientifold on $T^{4}$ and the D9-D5 system of Type IIB symplectic orientifold on $\widehat{T}^{4} / \mathbf{Z}_{2}$ is given by this Nahm transform.

\section{(iii) Orbibundle}

As the final example, we consider Type IIB string theory on orbifold $\mathbf{R}^{6} \times$ $T^{4} / \mathbf{Z}_{2}$ with $2 N$ D9-branes wrapped on $T^{4} / \mathbf{Z}_{2}$ and $k$ D5-branes at points on $T^{4} / \mathbf{Z}_{2}$. This system is represented by a rank $2 N$ orbibundle $(E, \varphi)$ on $T^{4} / \mathbf{Z}_{2}$ of instanton number $2 k$ with a self-dual connection $A$.

\section{Probing by a Wrapped D5-brane Pair}

We probe the system by a pair of D5-branes wrapped on $T^{4} / \mathbf{Z}_{2}$. The theory of lowlying modes on the probe can be analyzed in the same way as before. It is a $5+1$ dimensional $(1,0)$ supersymmetric $U(2)$ gauge theory on $\mathbf{R}^{2} \times T^{4}$ with $U(2 N)$ flavor symmetry where the eight supersymmetries are broken to half by constraints on the fields (written below) and by the instanton configuration $A_{\mu} \mathrm{d} x^{\mu}$ of the flavor group. The theory containes a $U(2)$ vector multiplet, an adjoint hypermultiplet and a hypermultiplet in the bifundamental representation $\left(\mathbf{2 N}, \mathbf{2}^{*}\right)$ of $U(2 N) \times U(2)$. The bosonic fields $X^{p}$ from the adjoint hypermultiplet and the $U(2)$ gauge field $a^{U(2)}$ (and their superpartners) are subject to the constraints

$$
\begin{aligned}
& X^{p}(-x)=\phi X^{p}(x) \phi, \\
& a_{\mu}^{U(2)}(-x)=-\phi a_{\mu}^{U(2)}(x) \phi, \quad a_{ \pm}^{U(2)}(-x)=\phi a_{ \pm}^{U(2)}(x) \phi,
\end{aligned}
$$

where $\phi$ is a $2 \times 2$ matrix as $\Phi$ in (3.10). The gauge transformations are also subject to the constraint $\phi g(x) \phi=g(-x)$. The hypermultiplet fields in $\left(\mathbf{2 N}, \mathbf{2}^{*}\right)$, the sections $Q^{\sigma \mathrm{a}}$ and $\Psi^{\mathrm{a}}$ of $E$ and $E \otimes S_{5+1}^{-}(\mathrm{a}=1,2$ are $U(2)$ gauge indices), are subject to the "half-hypermultiplet" conditions: $Q^{\sigma a}=$ $\phi_{\mathrm{b}}^{\mathrm{a}} \varphi Q^{\sigma \mathrm{b}}$ and

$$
\Psi^{\mathrm{a}}=\phi_{\mathrm{b}}^{\mathrm{a}} \varphi \Psi^{\mathrm{b}},
$$

where $\varphi Q$ and $\varphi \Psi$ are defined respectively by $(\varphi Q)(x)=\varphi_{-x} Q(-x)$ and $(\varphi \Psi)(x)=\Gamma^{1234} \varphi_{-x} \Psi(-x)$. These conditions are invariant under the supersymmetry (3.18) generated by the symplectic-Majorana-Weyl spinor $\xi_{\sigma}$ which is positive also in four dimensions $\Gamma^{1234} \xi_{\sigma}=\xi_{\sigma}$. 
At long distances, we obtain an effective $1+1$ dimensional theory with $(0,4)$ supersymmetry. Note that the gauge transformations and the \pm component of the gauge field that are constant along $T^{4}$ are in the $U(1) \times U(1)$ subgroup defined by the embedding

$$
\left(\mathrm{e}^{i s}, \mathrm{e}^{i t}\right) \mapsto \frac{1}{2}\left(\begin{array}{cc}
\mathrm{e}^{i s}+\mathrm{e}^{i t} & -\mathrm{e}^{i s}+\mathrm{e}^{i t} \\
-\mathrm{e}^{i s}+\mathrm{e}^{i t} & \mathrm{e}^{i s}+\mathrm{e}^{i t}
\end{array}\right) .
$$

Thus, the effective theory has $U(1) \times U(1)$ gauge symmetry with the gauge field $a_{ \pm}^{U(1) \times U(1)}$. The $T^{4}$ components of the gauge field reduces to scalar fields taking values in the moduli space of flat $U(2)$ connections on $T^{4}$ subject to the constraint (3.43). Such a flat $U(2)$ connection can be expressed as a constant gauge field of the form

$$
a_{\mu}^{U(2)}=i\left(\begin{array}{cc}
a_{\mu} & 0 \\
0 & -a_{\mu}
\end{array}\right) .
$$

Since there are (constrained) $U(2)$ gauge equivalence relations $a_{\mu} \equiv-a_{\mu} \equiv$ $a_{\mu}+\hat{n}_{\mu}\left(\hat{n} \in 2 \pi \Lambda^{*}\right)$, the moduli space is $\widehat{T}^{4} / \mathbf{Z}_{2}$. The adjoint hypermultiplet reduces to scalar multiplet taking values in the adjoint representation of the $U(1) \times U(1)$ subgroup. Away from the $\mathbf{Z}_{2}$ fixed point of $T^{4}$, the gauge group $U(1) \times U(1)$ is broken to its diagonal subgroup $U(1)$, and we obtain $(0,4)$ supersymmetric $U(1)$ gauge theory which has singlet scalar fields taking values in $\mathbf{R}^{4} \times \widehat{T}^{4} / \mathbf{Z}_{2}$. At each of the fixed points, $U(1) \times U(1)$ is unbroken and a new branch develops.

In any of these branches, massless fields also come from the "half-hypermultiplet" in the $\left(\mathbf{2 N}, \mathbf{2}^{*}\right)$ representation. They correspond to the fields on $T^{4}$ satisfying the equations as (2.7) where now

$$
D_{\mu}=\partial_{\mu}+A_{\mu}-{ }^{t} a_{\mu}^{U(2)}
$$

in which $a_{\mu}^{U(2)}$ is the flat $U(2)$ gauge field (3.46). Namely, they come from the zero modes of the Laplace and the Dirac operators on $T^{4}$ associated with $D_{\mu}^{( \pm a)}=\partial_{\mu}+A_{\mu} \mp i a_{\mu}$. Generically, there are nothing else than $2 k$ negativechirality zero modes for each of $\not^{(a)}$ and $\not^{(-a)}$. Let us expand the fermion $\Psi^{a}$ by the orthonormal base $\psi_{i}( \pm a)$ of the space of $\not D^{( \pm a)}$ zero modes as

$$
\Psi^{1}=\sum_{i} \psi_{i}(a) \otimes \lambda^{1 i}, \quad \Psi^{2}=\sum_{i} \psi_{i}(-a) \otimes \lambda^{2 i}
$$


where $\lambda^{\mathrm{a}}=\left(\lambda^{\mathrm{a} i}\right)$ are $1+1$ dimensional positive-chirality fermions. The lagrangian for $\Psi^{\mathrm{a}}$ then becomes

$$
\left(\overline{\lambda^{1}}, \overline{\lambda^{2}}\right)\left(\partial_{-}+\partial_{-} a_{\mu}\left(\begin{array}{cc}
\hat{A}^{\mu}(a) & 0 \\
0 & -\hat{A}^{\mu}(-a)
\end{array}\right)-{ }^{t} a_{-}^{U(1) \times U(1)}\right)\left(\begin{array}{c}
\lambda^{1} \\
\lambda^{2}
\end{array}\right)
$$

where $\hat{A}^{\mu}(a) \mathrm{d} a_{\mu}$ is the $U(2 k)$ connection of the bundle $\hat{E}$ over $\widehat{T}^{2}$ of $\not D^{(a)}$ zero modes defined as before. The bundle $\hat{E}_{ \pm}$have instanton number $2 N$ and $\hat{A}=\hat{A}^{\mu}(a) \mathrm{d} a_{\mu}$ has a self-dual curvature.

The fermions $\lambda^{\mathrm{a}}$ are constrained due to the "half-hypermultiplet" condition for $\Psi^{\mathrm{a}}$. It is easy to see that, if $\psi$ is a zero mode of $\not^{(a)}$, then $\varphi \psi$ (defined by $(\varphi \psi)(x)=\varphi_{-x} \psi(-x)$ ) is a zero mode of $\not^{(-a)}$. In particular $\varphi \psi_{i}(a)$ can be spanned by $\psi_{j}(-a)$ :

$$
\varphi \psi_{i}(a)=\psi_{j}(-a) \hat{\varphi}_{a i}^{j} .
$$

Then, the "half-hypermultiplet" condition requires

$$
\lambda^{2 i}=\hat{\varphi}_{a j}^{i} \lambda^{1 j}
$$

The linear map $\hat{\varphi}_{a}: \hat{E}_{a} \rightarrow \hat{E}_{-a}$ induced by $\psi \mapsto \varphi \psi$ defines a lift to $\hat{E}$ of the inversion of $\widehat{T}^{4}$. Indeed, it is involutive and unitary because $\varphi$ is. It is also easy to see that $\hat{A}$ preserves $\hat{\varphi}$. Thus, we have an orbibundle $(\hat{E}, \hat{\varphi})$ on $\widehat{T}^{4} / \mathbf{Z}_{2}$ with a connection $\hat{A}$.

The effective theory we have obtained is exactly the same as the effective theory of a D1-brane probing the system of $2 k$ D9-branes on orbifold $\widehat{T}^{4} / \mathbf{Z}_{2}$ which support the orbibundle $(\hat{E}, \hat{A}, \hat{\varphi})$. Indeed, (3.51) is nothing but the orientifold projection on 1-9 string modes. Thus, we can conclude that the Tduality mapping the D9-D5 system on orbifold $T^{4} / \mathbf{Z}_{2}$ to the D9-D5 system on the dual orbifold $\widehat{T}^{4} / \mathbf{Z}_{2}$ is represented by the transform $(E, A, \varphi) \mapsto$ $(\hat{E}, \hat{A}, \hat{\varphi})$ of the gauge field configurations. Since T-duality squares to the identity, the square of this transform must be identity. This can indeed be shown explicitly (see Appendix).

It should be possible to extend T-duality on orbifold $T^{4} / \mathbf{Z}_{2}$ to T-duality on its resolution - a smooth K3 surface. In [39], the transformation of Dbranes under T-duality on K3 surface was proposed using Mukai's Fourier transform. It is interesting to derive it using our argument and to see the relation to the construction of the present section. See also [37] for a related discussion which focuses on the B-field. 


\section{Reduction to Topology}

Recently, it was argued by Witten that D-brane charges take values in $\mathrm{K}$ theory groups of the space-time [15]. Since T-duality is an equivalence of string theories which sends D-branes to D-branes, (if the identification of D-brane charge as K-theory element is valid for any size of the space-time) it should induce an isomorphism of relevent K-theory groups. One is then interested in what this isomorphism is in general. In this section, we determine this for T-duality on four-torus by reducing to topology the Nahm transforms obtained in the previous sections. This serves as a warm-up for the next section where we will determine the isomorphisms for tori of other dimensions.

\subsection{D-branes, K-Theory, and Index Theory}

The basic assumption behind the identification of K-groups as D-brane charges [15] is that a $\mathrm{D} p$-brane and an anti- $\mathrm{D} p$-branes can annihilate by condensation of the tachyon field. ${ }^{1}$ D-branes located at a submanifold $W$ of the space-time support a complex vector bundle $E$ on $W$ with a connection. Likewise anti-D-branes at $W$ support another vector bundle $F$ with a connection. The tachyon field is a complex linear map $T: E \rightarrow F$ (and its conjugate $T^{\dagger}: F \rightarrow E$ ). If $T$ is everywhere at the minimum of the tachyon potential and hence is an isomorphism, then the system is considered to be equivalent to the vacuum; i.e. if $E$ and $F$ are isomorphic, the branes and anti-branes will annihilate. The set of pairs $(E, F)$ modulo an equivalence relation $(E, F) \equiv\left(E^{\prime}, F^{\prime}\right)$ when there are $H$ and $H^{\prime}$ such that $(E \oplus H, F \oplus H) \cong\left(E^{\prime} \oplus H^{\prime}, F^{\prime} \oplus H^{\prime}\right)$ forms the K-theory group $\mathrm{K}(W)$.

We have actually experienced in section 2 a phenomenon analogous to brane-anti-brane annihilation; it is the decoupling of brane-anti-brane pairs in the infra-red limit of the probe theory. We considered a vector bundle

\footnotetext{
${ }^{1}$ The tachyon is created by stretched strings and its condensation breaks the off-diagonal $U(1)$ subgroup of $U(1) \times U(1)$. There has been a puzzle about the fate of diagonal $U(1)$ which appears to remain unbroken $[40,15]$. However, it can disappear by confinement, namely, by condensation of the "magnetic tachyon" which is created by stretched (anti) $\mathrm{D}(p-2)$-branes and is charged under the $(p-2)$-form potential dual to the diagonal $U(1)$. See [41] for some of the details. In the present context, these are simply invisible sectors and will not be mentioned.
} 
$E$ on $T^{4}$ supported by D9-branes and probed the system with a D5-brane wrapped on $T^{4}$. The effective theory on the D5-brane, which is a $1+1$ dimensional theory with a tower of Kaluza-Klein modes, can be identified with the effective theory of a D1-brane probing the T-dualized system. The infinite Kaluza-Klein modes from the 5-9 hypermultiplet are interpreted as the strings stretched between the D1-brane and infinitely-many D9 or antiD9-branes of the T-dualized system. The fermion masses come from the Dirac operators on $T^{4}$ and are interpreted as the tachyon vevs of infinite D9 anti-D9 pairs. Massive modes are irrelevant at long distances and can be simply ignored. We can interpret this as the pair annihilation of the D9 and anti-D9-branes via tachyon condensation.

Now, as in [15], we can associate to this system of D9 and anti-D9-branes an element of an appropriate K-theory group which can be considered as the D-brane charge of the T-dualized system. Let $\mathcal{E}^{+}$and $\mathcal{E}^{-}$be the spaces of sections of negative and positive-chirality spinor bundles on $T^{4}$ coupled to $E$, as in section 2. The Dirac operator $\not D$ defines a complex linear map $\mathcal{D}: \mathcal{E}^{+} \rightarrow \mathcal{E}^{-}$and its conjugate $\mathcal{D}^{\dagger}: \mathcal{E}^{-} \rightarrow \mathcal{E}^{+}$. The spaces $\mathcal{E}^{+}$and $\mathcal{E}^{-}$ together with the operator $\mathcal{D}$ are parametrized by $a_{\mu} \in \widehat{T}^{4}$ and are considered as bundles over $\widehat{T}^{4}$ and a map between them. We have interpreted them as the bundles supported by the D9 and the anti-D9-branes and the tachyon field of the T-dualized system. Thus, the desired element of a K-theory group is " $\left(\mathcal{E}^{+}, \mathcal{E}^{-}\right)$" which belongs to $K\left(\widehat{T}^{4}\right)$.

One may wonder whether the fact that the bundles $\mathcal{E}^{+}$and $\mathcal{E}^{-}$are infinite-dimensional causes some trouble. However, since the massive modes are irrelevant (or since brane-anti-brane pair can annihilate via tachyon condensation), we can throw away the higher level modes and reduce the problem to finite dimension. Let us decompose $\mathcal{E}^{+}$and $\mathcal{E}^{-}$as $\mathcal{E}^{+}=\mathcal{E}_{0}^{+} \oplus \mathcal{E}_{1}^{+}$and $\mathcal{E}^{-}=\mathcal{E}_{0}^{-} \oplus \mathcal{E}_{1}^{-}$in such a way that $\mathcal{D}$ sends $\mathcal{E}_{i}^{+}$to $\mathcal{E}_{i}^{-}(i=0,1), \mathcal{E}_{0}^{+}$and $\mathcal{E}_{0}^{-}$ are finite-dimensional, and $\mathcal{D}: \mathcal{E}_{1}^{+} \rightarrow \mathcal{E}_{1}^{-}$is an isomorphism. Locally such a decomposition always exists as one can see, say, by taking the spectral decomposition and defining $\mathcal{E}_{1}^{+}$and $\mathcal{E}_{1}^{-}$as the modes whose Dirac eigenvalues do not vanish. If we could take such a decomposition globally on $\widehat{T}^{4}$, we would be able to regularize " $\left(\mathcal{E}^{+}, \mathcal{E}^{-}\right)$" by $\left(\mathcal{E}_{0}^{+}, \mathcal{E}_{0}^{-}\right)$defining an element of $K\left(\widehat{T}^{4}\right)$. Such a global decomposition does not always exist, but we can glue the local decompositions to obtain an element of $K\left(\widehat{T}^{4}\right)$ which restricts 
locally to $\left(\mathcal{E}_{0}^{+}, \mathcal{E}_{0}^{-}\right) .{ }^{2}$ This is actually exactly what the family index theory defines [6-8] as the index of $\mathcal{D}: \mathcal{E}^{+} \rightarrow \mathcal{E}^{-}$.

The operator $\mathcal{D}: \mathcal{E}^{+} \rightarrow \mathcal{E}^{-}$is defined as the Dirac operator $D^{+}:$ $\Gamma\left(S^{+}\right) \rightarrow \Gamma\left(S^{-}\right)$coupled to the family of connections $A_{\mu}-i a_{\mu}$ over $T^{4}$ parametrized by $a \in \widehat{T}^{4}$. Its index actually depends only on the topology of the bundle $\mathcal{V}$ on $T^{4} \times \widehat{T}^{4}$ which carries this family of connections (i.e. the bundle which has a connection which restricts on $T^{4} \times\{a\}$ to the connection $\left.A_{\mu}-i a_{\mu}\right)$. The index can therefore be denoted as ind $\left(D^{+}, \mathcal{V}\right)$. Thus, we need to find out what $\mathcal{V}$ is.

\section{The Poincaré Bundle}

The $U(1)$ connection $\partial_{\mu}-i a_{\mu}$ on $T^{4}$ is equivalent to $\partial_{\mu}-i\left(a_{\mu}+\hat{n}_{\mu}\right)$ for $\hat{n}_{\mu} \in 2 \pi \Lambda^{*}$ under the gauge transformation $\mathrm{e}^{i n x}$. Also, we recall from section 2 that the $U(1)$ gauge symmetry of the probe D5-brane implies that the section $\psi(x)$ of $S^{ \pm} \otimes E$ at $a \in\left(\mathbf{R}^{4}\right)^{*}$ should be identified as the section $\mathrm{e}^{i \hat{n} x} \psi(x)$ at $a+\hat{n} \in\left(\mathbf{R}^{4}\right)^{*}$. These motivate us to define a complex line bundle $\mathcal{P}$ over $T^{4} \times \widehat{T}^{4}$ as the quotient of the trivial bundle $T^{4} \times\left(\mathbf{R}^{4}\right)^{*} \times \mathbf{C}$ by the action of the lattice $2 \pi \Lambda^{*}$ given by

$$
\hat{n}:(x, a, c) \longmapsto\left(x, a+\hat{n}, \mathrm{e}^{i \hat{n} x} c\right) .
$$

We call this the Poincaré bundle. The gauge potential $-i a_{\mu} \mathrm{d} x^{\mu}$ on $T^{4} \times\left(\mathbf{R}^{4}\right)^{*}$ is invariant under the transformation (4.1) and defines a connection $\omega$ of $\mathcal{P}$ that restricts on $T^{4} \times\{a\}$ to the flat connection $\partial_{\mu}-i a_{\mu}$ of the trivial bundle. When restricted to $\{x\} \times \widehat{T}^{4}$, the connection $\omega$ yields a flat connection on $\widehat{T}^{4}$ which is equivalent to $\partial / \partial a_{\mu}+i x^{\mu}$. However, $\omega$ is not flat over $T^{4} \times \widehat{T}^{4}$ and has a curvature $\mathrm{d} \omega$ with a first Chern class

$$
c_{1}(\mathcal{P})=\sum_{\mu=1}^{4} \frac{\mathrm{d} a_{\mu} \wedge \mathrm{d} x^{\mu}}{2 \pi}=\sum_{i=1}^{4} \widehat{\eta}^{i} \wedge \eta_{i}
$$

where $\eta_{i}$ and $\widehat{\eta}^{i}$ are basis of $H^{1}\left(T^{4}, \mathbf{Z}\right)$ and $H^{1}\left(\widehat{T}^{4}, \mathbf{Z}\right)$ respectively (more precisely, their image under the embedding of the integral to the real cohomology of the torus).

\footnotetext{
${ }^{2}$ Here is the construction: Collecting the spaces $\mathcal{E}_{0}^{-}$defined locally, using cut-off functions we obtain a finite-dimensional vector bundle $V$ over $\widehat{T}^{4}$ with a map $f: V \rightarrow \mathcal{E}^{-}$such that $\mathcal{D} \oplus f: \mathcal{E}^{+} \oplus V \rightarrow \mathcal{E}^{-}$is surjective everywhere. The kernel of $\mathcal{D} \oplus f$ has a constant rank and defines a vector bundle over $\widehat{T}^{4}$. Then, we define " $\left(\mathcal{E}^{+}, \mathcal{E}^{-}\right)$" by $(\operatorname{Ker}(\mathcal{D} \oplus f), V)$.
} 


\section{The Isomorphism $\mathrm{K}\left(T^{4}\right) \cong \mathrm{K}\left(\widehat{T}^{4}\right)$}

The tensor product $E \otimes \mathcal{P}$ has a connection $A \otimes 1+1 \otimes \omega$ that restricts on $T^{4} \times\{a\}$ to the connection $A_{\mu}-i a_{\mu}$. Also, $\mathcal{E}^{+}$and $\mathcal{E}^{-}$are vector bundles whose fibres at $a \in \widehat{T}^{4}$ are given by $\mathcal{E}_{a}^{+}=\Gamma\left(T^{4},\left.S^{+} \otimes E \otimes \mathcal{P}\right|_{a}\right)$ and $\mathcal{E}_{a}^{-}=$ $\Gamma\left(T^{4},\left.S^{-} \otimes E \otimes \mathcal{P}\right|_{a}\right)$. Therefore, $E \otimes \mathcal{P}$ is the desired bundle over $T^{4} \times \widehat{T}^{4}$ that carries the family of connections defining the operator $\mathcal{D}: \mathcal{E}^{+} \rightarrow \mathcal{E}^{-}$.

Thus, we find that the T-duality transformation of the D-brane charge is given by $E \mapsto E \otimes \mathcal{P} \mapsto$ ind $\left(D^{+}, E \otimes \mathcal{P}\right)$, which can be considered as the image of $(E, 0) \in K\left(T^{4}\right)$ under the composition of the maps of K-theory groups:

$$
\mathrm{K}\left(T^{4}\right) \stackrel{\otimes \mathcal{P}}{\longrightarrow} \mathrm{K}\left(T^{4} \times \widehat{T}^{4}\right) \stackrel{\text { ind } D^{+}}{\longrightarrow} \mathrm{K}\left(\widehat{T}^{4}\right) .
$$

By abuse of language, we shall denote ind $\left(D^{+}, E \otimes \mathcal{P}\right)$ by $\widehat{E}$. Since the Nahm transform squares to the identity transform of instantons, the above map, which is the topological reduction of the Nahm transform, must also square to the identity of $\mathrm{K}\left(T^{4}\right)$. Namely, the inverse of (4.3) is given by

$$
\mathrm{K}\left(T^{4}\right) \stackrel{\text { ind } \widehat{D}^{+}}{\longleftarrow} \mathrm{K}\left(T^{4} \times \widehat{T}^{4}\right) \stackrel{\otimes \widehat{\mathcal{P}}}{\longleftarrow} \mathrm{K}\left(\widehat{T}^{4}\right),
$$

where $\widehat{D}^{+}$is the Dirac operator of $\widehat{T}^{4}$ and $\widehat{\mathcal{P}}$ is the dual of $\mathcal{P}$ with connection $-\omega$.

To compute the index, we use the formula

$$
\operatorname{ch}\left(\operatorname{ind}\left(D^{+}, \mathcal{V}\right)\right)=\int_{X} \operatorname{ch}(\mathcal{V}) \widehat{A}(X)
$$

which holds for the Dirac operator $D^{+}: \Gamma\left(S^{+}\right) \rightarrow \Gamma\left(S^{-}\right)$on any evendimensional spin manifold $X$ coupled to any family of connections carried by a bundle $\mathcal{V}$ over $X \times Y$ where $Y$ is a compact parameter space. Here, $\widehat{A}(X)$ is the A-roof genus of $X$ which belongs to $H^{*}(X, \mathbf{Q})$ and ch is the Chern character, which is a group homomorphism

$$
\operatorname{ch}: \mathrm{K}(X) \rightarrow H^{\text {even }}(X, \mathbf{Q})
$$

defined for a general topological space $X$. Note that $\mathrm{ch}_{0}=\operatorname{rank}, \mathrm{ch}_{1}=c_{1}$, and $\mathrm{ch}_{2}=c_{1}^{2} / 2-c_{2}$ etc.

In the present case, we have (see the next section)

$$
\mathrm{K}\left(T^{4}\right) \cong \mathbf{Z}^{8}
$$


and the Chern character maps this isomorphically to the subgroup $H^{\text {even }}\left(T^{4}, \mathbf{Z}\right) \cong$ $\mathbf{Z}^{8}$ of $H^{\text {even }}\left(T^{4}, \mathbf{Q}\right) \cong \mathbf{Q}^{8}$ where $H^{0}\left(T^{4}, \mathbf{Z}\right) \cong \mathbf{Z}, H^{2}\left(T^{4}, \mathbf{Z}\right) \cong \mathbf{Z}^{6}$ (generated by $\left.\eta^{i} \eta^{j}\right), H^{4}\left(T^{4}, \mathbf{Z}\right) \cong \mathbf{Z}$ (generated by the volume form). In particular, no information is lost by looking only at the Chern character. Since we have $\mathcal{V}=$ $E \otimes \mathcal{P}$ and $\widehat{A}\left(T^{4}\right)=1$ the character of the index is $\operatorname{ch}(\widehat{E})=\int_{T^{4}} \operatorname{ch}(E) \operatorname{ch}(\mathcal{P})$. Since $\mathcal{P}$ is a line bundle we have $\operatorname{ch}(\mathcal{P})=\mathrm{e}^{c_{1}(\mathcal{P})}$. Then, (4.2) shows that the Chern numbers of $E$ are related to that of the index $\widehat{E}$ as

$$
\begin{aligned}
& \operatorname{rank}(\widehat{E})=\operatorname{ch}_{2}(E), \\
& c_{1}(\widehat{E})=-\sigma\left(c_{1}(E)\right) \\
& \operatorname{ch}_{2}(\widehat{E})=\operatorname{rank}(E)
\end{aligned}
$$

where $\sigma$ is a map of $H^{2}\left(T^{4}, \mathbf{Z}\right)$ to $H^{2}\left(\widehat{T}^{4}, \mathbf{Z}\right)$ sending $\eta^{i} \eta^{j}$ to $\frac{1}{2} \epsilon_{i j k l} \widehat{\eta}^{k} \widehat{\eta}^{l}$. In particular, the map $\mathrm{K}\left(T^{4}\right) \rightarrow \mathrm{K}\left(\widehat{T}^{4}\right)$ given by (4.3) is an isomorphism.

\subsection{Orthogonal/Symplectic (Orienti)bundles and $\mathrm{Z}_{2}$ Orbibun- dles}

We next find the map of D-brane charges under the T-duality on $T^{4}$ in the presence of orientifold/orbifold projection, by considering the topological reduction of the Nahm transforms constructed in section 3 .

\section{Structures of the Poincaré Bundle}

The bundle $\mathcal{P}$ and the connection $\omega$ has two basic properties which are useful for the present discussion (and which we have actually implicitly used in the construction of the Nahm transform). One is the structure of an orthogonal orientibundle. We note that the anti-linear involution

$$
I:(x, a, c) \mapsto(x,-a, \bar{c})
$$

of the trivial bundle over $T^{4} \times\left(\mathbf{R}^{4}\right)^{*}$ commutes with the action (4.1) of $2 \pi \Lambda^{*}$, and hence defines and orthogonal structure on $\mathcal{P}$ over $(x, a) \mapsto(x,-a)$. We also note that the connection $\omega$ coming from the gauge potential $-i a_{\mu} \mathrm{d} x^{\mu}$ is invariant under this $\mathbf{Z}_{2}$ action. Thus, $(\mathcal{P}, \omega, I)$ becomes an orthogonal orientibundle with a connection over $T^{4} \times \widehat{T}^{4}$ with respect to the inversion of the $\widehat{T}^{4}$ factor. The other is the structure of an orbibundle. The involution

$$
\varphi:(x, a, c) \mapsto(-x,-a, c)
$$


obviously commutes with the $2 \pi \Lambda^{*}$ action and preserves the connection $\omega$. Thus, $(\mathcal{P}, \omega, \varphi)$ is an orbibundle with a connection over $T^{4} \times \widehat{T}^{4}$ with respect to the total inversion.

\section{Relevant K-theory Groups}

As we have seen in section 3.3, in theories with orientifold/orbifold projection the Chan-Paton bundles on D9-branes have various extra structures. Accordingly, the relevant K-theory groups vary.

The K-theory group for orthogonal bundles over a space $X$ is the KOtheory group of $X$ denoted by $\mathrm{KO}(X)$. That for symplectic bundles is likewise denoted by $\operatorname{KSp}(X)$. Now let $Y$ be a space with an involution $\sigma$. Then, the K-theory group for orthogonal orientibundles over $Y$ with respect to $\sigma$ is what is known as KR-theory group and is denoted as $\mathrm{KR}(Y)$. We can also define $\mathrm{K}$-theory group for symplectic orientibundles over $Y$ with respect to $\sigma$. We shall denote it by $\operatorname{KpR}(Y) .{ }^{1}$ Finally, the K-theory for $\mathbf{Z}_{2}$ orbibundles over $Y$ with respect to $\sigma$ is given by the $\mathbf{Z}_{2}$-equivariant $\mathrm{K}$-theory and the group is denoted by $\mathrm{K}_{\mathbf{Z}_{2}}(Y)$.

Note that orthogonal (orienti)bundles and $\mathbf{Z}_{2}$-orbibundles are closed under tensor product. Therefore $\mathrm{KO}(X), \mathrm{KR}(Y)$ and $\mathrm{K}_{\mathbf{Z}_{2}}(Y)$ become rings. However, symplectic (orienti)bundles are not.closed and thus $\mathrm{KSp}(X)$ and $\mathrm{KpR}(Y)$ has no ring structure. Instead, the tensor product of two symplectic (orienti)bundles is an orthogonal (orienti)bundle, and the tensor product of an orthogonal and a symplectic (orienti)bundles is a symplectic (orienti)bundle. Thus, the sums $\mathrm{KO}(X) \oplus \mathrm{KSp}(X)$ and $\mathrm{KR}(Y) \oplus \mathrm{KpR}(Y)$ become rings.

\section{(i) $\mathrm{KO}\left(T^{4}\right) \cong \mathrm{KpR}\left(\widehat{T}^{4}\right)$}

We first consider the $S O$-type orientifold and find the transformation of $\mathrm{D}$ brane charges under T-duality on four-torus. As we have seen in section 3, D9-branes in Type I string theory support orthogonal bundles while those in Type IIB orientifold on $\widehat{T}^{4} / \mathbf{Z}_{2}$ support symplectic orientibundles over $\widehat{T}^{4}$ with respect to the inversion. Thus, we must find a map from $\operatorname{KO}\left(T^{4}\right)$

\footnotetext{
${ }^{1}$ The same group appears in [19] and is named as $K H(Y)$.
} 
to $\operatorname{KpR}\left(\widehat{T}^{4}\right)$ and back. As in the previous subsection, we can find this by taking the topological reduction of the Nahm transform between orthogonal bundles and symplectic orientibundles obtained in section 3 . We recall that the transform is based on the Nahm transform for the underlying unitary bundles. Thus, we basically follow the maps (4.3) and (4.4).

Since the Poincaré bundle $\mathcal{P}$ has an orthogonal structure over the inversion of $\widehat{T}^{4}$, the tensor product of an orthogonal bundle over $T^{4}$ (pulled back to $T^{4} \times \widehat{T}^{4}$ ) and $\mathcal{P}$ defines an orthogonal orientibundle over $T^{4} \times \widehat{T}^{4}$ with respect to the inversion of the $\widehat{T}^{4}$ factor. Thus, $\otimes \mathcal{P}$ defines a map from $\mathrm{KO}\left(T^{4}\right)$ to $\mathrm{KR}\left(T^{4} \times \widehat{T}^{4}\right)$. On the other hand, the spinor bundles $S^{ \pm}$on $T^{4}$ (or on any four-dimensional spin manifold) has a symplectic structure coming from the charge conjugation. Thus, tensoring an orthogonal orientibundle with $S^{ \pm}$(pulled back to $T^{4} \times \widehat{T}^{4}$ ) makes a symplectic orientibundle. Then, the index of the Dirac operator on $T^{4}$ becomes a symplectic orientibundle over $\widehat{T}^{4}$. Thus, ind $D^{+}$defines a map from $\operatorname{KR}\left(T^{4} \times \widehat{T}^{4}\right)$ to $\operatorname{KpR}\left(\widehat{T}^{4}\right)$. By composition, we obtain a map

$$
\mathrm{KO}\left(T^{4}\right) \stackrel{\otimes \mathcal{P}}{\longrightarrow} \mathrm{KR}\left(T^{4} \times \widehat{T}^{4}\right) \stackrel{\text { ind } D^{+}}{\longrightarrow} \mathrm{KpR}\left(\widehat{T}^{4}\right) .
$$

In section 3 , we have seen that the symplectic structure over the inversion of $\widehat{T}^{4}$ obtained this way is exactly the same as the one for the T-dualized system. Thus, (4.13) is the desired map for the T-duality transformation of the D-branes charges.

The inverse map can be obtained in a similar way. We only have to note that the dual Poincaré bundle $\widehat{\mathcal{P}}$ also has an orthogonal structure over the inversion of the $\widehat{T}^{4}$ factor and that the spinor bundles $\widehat{S}^{ \pm}$on $\widehat{T}^{4}$ has a symplectic structure over the inversion coming from the charge conjugation followed by the multiplication by $\Gamma^{1234}$. The result is

$$
\mathrm{KO}\left(T^{4}\right) \stackrel{\text { ind } \widehat{D}^{+}}{\longleftarrow} \mathrm{KpR}\left(T^{4} \times \widehat{T}^{4}\right) \stackrel{\otimes \widehat{\mathcal{P}}}{\longleftarrow} \mathrm{KpR}\left(\widehat{T}^{4}\right) .
$$

The two maps (4.13) and (4.14) must be the inverse of each other, since the Nahm transforms are. In particular, these must be isomorphisms of groups. Indeed these groups are isomorphic (see the next section)

$$
\mathrm{KO}\left(T^{4}\right)=\mathbf{Z} \oplus \mathbf{Z}_{2}^{\oplus 4} \oplus \mathbf{Z}_{2}^{\oplus 6} \oplus \mathbf{Z}=\mathrm{KpR}\left(\widehat{T}^{4}\right) .
$$




\section{(ii) $\operatorname{KSp}\left(T^{4}\right) \cong \operatorname{KR}\left(\widehat{T}^{4}\right)$}

We next consider T-duality on four-torus of the $S p$-type orientifold. As before, we obtain the map of D-brane charges, from $\operatorname{KSp}\left(T^{4}\right)$ to $\operatorname{KR}\left(\widehat{T}^{4}\right)$ and back, by topological reduction of the Nahm transform of section 3 . We use the orthogonal structure of $\mathcal{P}(\widehat{\mathcal{P}})$ over the inversion of the $\widehat{T}^{4}$ factor and the symplectic structure of the spinor bundles $S^{ \pm}\left(\widehat{S}^{ \pm}\right)$. The result is

$$
\mathrm{KSp}\left(T^{4}\right) \stackrel{\otimes \mathcal{P}}{\longrightarrow} \mathrm{KpR}\left(T^{4} \times \widehat{T}^{4}\right) \stackrel{\text { ind } D^{+}}{\longrightarrow} \mathrm{KR}\left(\widehat{T}^{4}\right),
$$

and the inverse is

$$
\operatorname{KSp}\left(T^{4}\right) \stackrel{\text { ind } \widehat{D}^{+}}{\longleftarrow} \operatorname{KR}\left(T^{4} \times \widehat{T}^{4}\right) \stackrel{\otimes \widehat{\mathcal{P}}}{\longleftarrow} \mathrm{KR}\left(\widehat{T}^{4}\right) .
$$

The two groups are indeed isomorphic

$$
\mathrm{KSp}\left(T^{4}\right)=\mathbf{Z} \oplus \mathbf{Z}=\mathrm{KR}\left(\widehat{T}^{4}\right) .
$$

(iii) $\mathrm{K}_{\mathbf{Z}_{2}}\left(T^{4}\right) \cong \mathrm{K}_{\mathbf{Z}_{2}}\left(\widehat{T}^{4}\right)$

Finally we obtain the map from $\mathrm{K}_{\mathbf{Z}_{2}}\left(T^{4}\right)$ to $\mathrm{K}_{\mathbf{Z}_{2}}\left(\widehat{T}^{4}\right)$ by reducing the Nahm transform. What we use here is the fact that the Poincaré bundle $\mathcal{P}$ has a complex linear lift of the inversion of $T^{4} \times \widehat{T}^{4}$ and that the spinor bundles $S^{ \pm}$ also have a linear lift of the inversion given by the multiplication by $\Gamma^{1234}$. Then, the map is easily obtained. It is

$$
\mathrm{K}_{\mathbf{Z}_{2}}\left(T^{4}\right) \stackrel{\otimes \mathcal{P}}{\longrightarrow} \mathrm{K}_{\mathbf{Z}_{2}}\left(T^{4} \times \widehat{T}^{4}\right) \stackrel{\text { ind } D^{+}}{\longrightarrow} \mathrm{K}_{\mathbf{Z}_{2}}\left(\widehat{T}^{4}\right) .
$$

The inverse map is similar.

\section{Generalizations}

In the final section, we identify the isomorphism of appropriate K-theory groups that realizes the map of D-brane charges under T-duality on torus of arbitrary dimensions. 


\subsection{Relevant K-theory Groups}

As argued in [15] and as we have experienced, D-branes that can be represented as bound states of $\mathrm{D} p$-branes and anti- $\mathrm{D} p$-branes wrapped on a $(p+1)$-dimensional submanifold $W$ are classified by an appropriate K-theory group of $W$. In string thoery, it seems common that any D-brane can be represented as a bound state of the highest dimensional D-branes (and antiD-branes) filling the ten-dimensional space-time $X$. Therefore, the entire D-brane charges of a string theory on $X$ can be classified by an appropriate K-theory group of the full space-time $X$.

The relevant $\mathrm{K}$-theory group for Type IIB string theory on $X$ is $\mathrm{K}(X)$ while it is $\operatorname{KO}(X)$ for Type I string on $X$ [15]. It was also proposed in [15] that D-brane charge in Type IIA string theory takes values in $\mathrm{K}^{-1}(X)$ - the subgroup of $\mathrm{K}\left(S^{1} \times X\right)$ consisting of elements that are trivial on $z_{0} \times X$ where $z_{0}$ is a point in $S^{1}$. This proposal was supported by Horava in [17]. He first argued that any Type IIA (BPS) D-brane can possibly be represented as a certain bound state of the unstable non-supersymmetric D9-branes $[42,17]$ and then identified the Chan-Paton bundle $E$ with the tachyon field $T: E \rightarrow E$ of D9-branes as the pair $\left(E, \mathrm{e}^{i T}\right)$ which determines an element of $\mathrm{K}^{-1}(X)$ via another version of the definition of $\mathrm{K}^{-1}(X)$. See [19] for another discussion.

However, the precise K-theory group has not yet been identified for Type II orientifold except for the cases where one knows the Chan-Paton bundles on ninebranes; for example it is $\mathrm{KR}(X)\left(\mathrm{KR}^{-1}(X)\right)$ when the Chan-Paton bundles on D9 and anti-D9-branes (IIA ninebranes) are orthogonal orientibundles $[15]([17]) .{ }^{1}$ Here, we consider the $\mathbf{Z}_{2}$ orientifold group generated by an involution $\tau$ of the space-time $X$ times the worldsheet orientation reversal. $^{2} \mathrm{D} p$-branes on top of an $\mathrm{O} p$-plane $W$ support orthogonal Chan-Paton bundle over $W$ if $W$ is of $S O$-type while they support symplectic bundle over

\footnotetext{
${ }^{1}$ It was also proposed in [15] that it is $\mathrm{KR}_{ \pm}(X)$ for a different kind of Chan-Paton bundles. K-theory for orientifolds was also studied in [19] with an extension to symplectic Chan-Paton bundles.

${ }^{2}$ The generator is $(-1)^{c_{p} F_{L}} \tau \Omega$ with $c_{p}=\frac{(9-p)(8-p)}{2}$ in the standard notation, where we assume that there are $\mathrm{O} p$-planes for a single $p$ - odd for IIB, even for IIA $\left((-1)^{c_{p} F_{L}}\right.$ is for the group to be $\left.\mathbf{Z}_{2}\right)$. As before, an $\mathrm{O} p$-plane $W$ is a $(p+1)$-dimensional submanifold of $X$ consisting of fixed points of the involution $\tau$ which acts near $W$ as the sign flip of $(9-p)$ normal coordinates. It is of $S O$-type or $S p$-type depending on the sign of the surrounding $\mathbf{R} \mathbf{P}^{2}$ diagram of fundamental string.
} 
$W$ if $W$ is of $S p$-type. Away from orientifold planes, physics in the quotient space $X / \mathbf{Z}_{2}$ is locally the same as the underlying Type II string theory and in particular D-branes locally support unitary bundles. Thus, the relevant K-theory group of $X$ must satisfy the two conditions:

(i) The group corresponding to D-branes localized on an orientifold plane $W$ must be $\mathrm{KO}(W)$ or $\mathrm{KSp}(W)$ if $W$ is of $S O$-type or of $S p$-type respectively. (ii) For a region $R$ in $X / \mathbf{Z}_{2}$ covered by two separate copies of $R$ in $X$, the group corresponding to D-branes in $R$ must be $\mathrm{K}(R)$ for IIB and $\mathrm{K}^{-1}(R)$ for IIA orientifold.

We shall propose K-groups which satisfy these conditions. In order to present it, we need to introduce some notions and facts in K-theory. See [43-45] for details.

\section{The Groups $\mathrm{KR}^{-n}(Y)$ and $\mathrm{KpR}^{-n}(Y)$}

Let $Y$ be a space with an involution $\sigma$. Let $D^{p, q}$ be the unit disc in $\mathbf{R}^{p+q}$ on which we let the involution act trivially on the first $p$ coordinates but by sign-flip on the last $q$ coordinates. We consider orthogonal orientibundles over $D^{p, q} \times Y$ with respect to the involution acting on $Y$ by $\sigma$ and on $D^{p, q}$ as dictated above. We define the group $\mathrm{KR}^{-p,-q}(Y)=\mathrm{KR}\left(D^{p, q} \times Y, \partial D^{p, q} \times\right.$ $Y$ ) as the KR-group for bundles over $D^{p, q} \times Y$ that vanish on $\partial D^{p, q} \times Y$. Here "bundle over $A$ that vanishes on $B \subset A$ " stands for a pair $(E, F)$ where $E$ and $F$ are bundles over $A$ that are isomorphic on $B,\left.\left.E\right|_{B} \cong F\right|_{B}$. Replacing "orthogonal" by "symplectic" we obtain the group $\mathrm{KpR}^{-p,-q}(Y)$. We denote $\mathrm{KR}^{-n}(Y)=\mathrm{KR}^{-n, 0}(Y)$ and $\mathrm{KpR}^{-n}(Y)=\mathrm{KpR}^{-n, 0}(Y)$. The following relations between these groups are known as Bott periodicity

$$
\begin{aligned}
& \mathrm{KR}^{-p,-q}=\mathrm{KR}^{-p-1,-q-1}, \\
& \mathrm{KR}^{-n}=\mathrm{KR}^{-n-8} \\
& \mathrm{KpR}^{-n}=\mathrm{KR}^{-n \pm 4} .
\end{aligned}
$$

Using these, we define $\mathrm{KR}^{n}$ etc for $n>0$. We see that any of these groups is equal to $\mathrm{KR}^{-n}$ for some $n$. We also define $\mathrm{KR}^{-n}(Y, Z)$ for a $\mathbf{Z}_{2}$-invariant subspace $Z \subset Y$ as the $\mathrm{KR}^{-n}$-group for bundles over $Y$ that vanish on $Z{ }^{3}$

\footnotetext{
${ }^{3}$ More precisely, $\mathrm{KR}^{-n}(Y, Z)$ is the KR-group for bundles over $S^{n} \wedge(Y / Z)$ that vanish on the base point, where $A \wedge B$ is the smash product defined by $(A \times B) /\left(\left(A \times b_{0}\right) \cup\left(a_{0} \times B\right)\right)$ (see [43]).
} 
When the involution $\sigma$ acts trivially on $Y$, we have $\mathrm{KR}(Y)=\mathrm{KO}(Y)$ and $\operatorname{KpR}(Y)=\mathrm{KSp}(Y)$, and we define $\mathrm{KO}^{-n}(Y):=\mathrm{KR}^{-n}(Y)$ and $\mathrm{KSp}^{-n}(Y):=$ $\mathrm{KpR}^{-n}(Y)$. When $Y$ is a disjoint union of two copies of a space $R$ and the involution $\sigma$ is the exchange of the copies, we have $\mathrm{KR}^{-n}(Y)=\mathrm{K}^{-n}(R)$ where $\mathrm{K}^{-n}$ is defined in a similar way and obeys Bott periodicity $\mathrm{K}^{-n}=$ $\mathrm{K}^{-n-2}$ so that $\mathrm{K}^{-n}=\mathrm{K}$ for even $n$ and $\mathrm{K}^{-n}=\mathrm{K}^{-1}$ for odd $n$.

\section{The proposal}

We now propose the relevant K-theory group: D-brane charges of Type II orientifold on $X / \mathbf{Z}_{2}$ are classified by

$$
\begin{aligned}
& \mathrm{KR}^{-(9-p)}(X) \text { if there are } \mathrm{O} p \text {-planes of } S O \text {-type only, } \\
& \mathrm{KpR}^{-(9-p)}(X) \text { if there are } \mathrm{O} p \text {-planes of } S p \text {-type only. }
\end{aligned}
$$

Let us show that this proposal satisfies the conditions (i) and (ii). We only consider $S O$-type orientifold (since $S p$-type orientifold is similar) and we put $n=9-p$. Condition (ii) is easy to check by using the relation noted above, $\mathrm{KR}^{-n}(R \sqcup R)=\mathrm{K}^{-n}(R)$, and the fact that $n$ is even ( $p$ odd) for IIB and $n$ is odd ( $p$ even) for IIA.

To test (i), let $W$ be an orientifold $p$-plane. For the moment, we assume that the normal bundle of $W$ in $X$ is trivial so that a neighborhood of $W$ in $X$ looks like a product space $D^{0, n} \times W$ on which $\tau$ acts on the sign-flip of the disc factor. From our proposal, the group corresponding to D-branes localized on $W$ can be identified as $\mathrm{KR}^{-n}\left(D^{0, n} \times W, \partial D^{0, n} \times W\right)$ which is the $\mathrm{KR}^{-n}$-group for bundles over $D^{0, n} \times W$ that vanishes on $\partial D^{0, n} \times W$. Namely, the KRgroup for bundles over $D^{n, 0} \times D^{0, n} \times W$ that vanishes on $\partial D^{n, 0} \times D^{0, n} \times W$ and $D^{n, 0} \times \partial D^{0, n} \times W$, i.e. on $\partial\left(D^{n, 0} \times D^{0, n}\right) \times W$. Since $D^{n, 0} \times D^{0, n} \cong D^{n, n}$ this is identified with $\mathrm{KR}\left(D^{n, n} \times W, \partial D^{n, n} \times W\right)=\mathrm{KR}^{-n,-n}(W)$. By the Bott periodicity (5.1), this is equal to $\mathrm{KR}(W)$ which is indeed $\mathrm{KO}(W)$ since the involution acts trivially on $W$. Even when the normal bundle is non-trivial, this is true at least locally. Glueing such local realtions is a generalization of the proof of Thom isomorphism theorem, and one can show that this is also true globally when the normal bundle has a spin structure (see Section 2.7 of [43] or Section 12 of [47]). The same remark applies also to the next paragraph as well. 
The proposed group also has another desirable property that could have been added to the conditions (i) and (ii). Before showing it, we comment on a related fact about the condition (i) itself. When $\mathrm{D}(p-4)$-branes are wrapped on a submanifold $W^{\prime}$ of an $\mathrm{O} p$-plane $W$ of $S O$-type, they support symplectic bundle over $W^{\prime}$. Thus, D-branes localized in $W^{\prime}$ must be classified by $\mathrm{KSp}\left(W^{\prime}\right)$. Since a neighborhood of $W^{\prime}$ in $W$ locally looks like $D^{4} \times$ $W^{\prime}$, the condition (i) shows that the relevant group is $\mathrm{KO}\left(D^{4} \times W^{\prime}, \partial D^{4} \times\right.$ $\left.W^{\prime}\right)$ which can be identified with $\mathrm{KO}^{-4}\left(W^{\prime}\right)$. This is indeed $\mathrm{KSp}\left(W^{\prime}\right)$ by Bott periodicity (5.3). Now we go back to our proposal. If $W^{\prime \prime}$ is a $\mathbf{Z}_{2^{-}}$ invariant submanifold of $X$ of dimension $p+5$ including all the $\mathrm{O} p$-planes (of $S O$-type), as we have seen in section $3, \mathrm{D}(p+4)$-branes wrapped on $W^{\prime \prime}$ support symplectic orientibundle over $W^{\prime \prime}$. Thus, D-branes localized in $W^{\prime \prime}$ must be classified by $\mathrm{KpR}\left(W^{\prime \prime}\right)$. In fact, a neighborhood of $W^{\prime \prime}$ in $X$ looks locally like $D^{0,5-p} \times W^{\prime \prime}$ and the relevant $\mathrm{K}$-group is identified as $\mathrm{KR}^{-(9-p)}\left(D^{0,5-p} \times W^{\prime \prime}, \partial D^{0,5-p} \times W^{\prime \prime}\right)$. As in the test of (i), this is equal to $\mathrm{KR}^{-(9-p),-(5-p)}\left(W^{\prime \prime}\right)$ which is indeed $\mathrm{KR}^{-4}\left(W^{\prime \prime}\right)=\mathrm{KpR}\left(W^{\prime \prime}\right)$ by Bott periodicity (5.1), (5.3).

We have shown that our proposal does satisfy all three requirements ((i), (ii) and another) but we have not shown that it is the only possibility. In the next subsection, we will show that it is the one that naturally appears when applying T-duality to Type I string theory (for $S O$-type orientifold). 4

Note that the proposal does not cover the most general cases. For example, there are orientifolds including $S O$-type and $S p$-type O-planes at the same time. There could also be cases where there are O-planes of mixed dimensions. In this paper, we do not attempt to specify the relevant K-theory group for such exotic (but sometimes interesting) models.

\subsection{The T-duality Isomorphism}

Now we identify the isomorphism of K-groups that realizes T-duality on $n$-torus.

We start with the basic example of $n=1$. Consider a D-brane configuration in Type IIB string theory on $T^{1} \times M$ where $M$ is a nine-dimensional

\footnotetext{
${ }^{4}$ Chronologically, that is the way I found the proposal.
} 
manifold. We represent it as a bound state of D9 and anti-D9-branes which support Chan-Paton bundles $E^{ \pm}$with connections $A_{M}^{ \pm}$and the tachyon field $T: E^{+} \rightarrow E^{-}$. We probe it with a D1-brane wrapped on $T^{1}$. Then, KaluzaKlein reduction of the probe theory is identified with the quantum mechanics of a D0-brane probing the T-dualized system on $\widehat{T}^{1} \times M$. We coordinatize $T^{1}$ and $\widehat{T}^{1}$ by $x$ and $a$ respectively. Mass matrix of the fermions in the quantum mechanics originating from 1-9 and 1- $\overline{9}$ strings are given by the operator

$$
\mathcal{D}=\left(\begin{array}{cc}
D_{+} & -T^{\dagger} \\
T & -D_{-}
\end{array}\right)
$$

Here $D_{ \pm}=\partial_{x}+A_{x}^{ \pm}-i a$ is the Dirac operator $D$ on $T^{1}$ coupled to the family of connections $A_{x}^{ \pm}-i a$ carried by $E^{ \pm} \otimes \mathcal{P}$ where $\mathcal{P}$ is the Poincaré bundle over $T^{1} \times \widehat{T}^{1}$ with curvature $-i \mathrm{~d} a \wedge \mathrm{d} x$. This is interpreted as the tachyon field of Type IIA ninebranes of the T-dualized system.

For instance, consider (unphysical) Type IIB string theory on $T^{1} \times \mathbf{R}^{9}$ with a single D9-brane with Wilson line $i a^{0} \mathrm{~d} x$ where $x$ is the coordinate on $T^{1}$ with periodicity $x \equiv x+1$. Then the Dirac operator on $T^{1}$ for the fermion of the probe D1-brane is $\mathcal{D}(a)=\partial_{x}+i a^{0}-i a$. Kaluza-Klein modes on $T^{1}$ consist of functions $\mathrm{e}^{i \hat{n} x}, \hat{n} \in 2 \pi \mathbf{Z}$, with $\mathcal{D}(a)$ eigenvalues $-i\left(a-\left(a^{0}+\hat{n}\right)\right)$. One interpretation of these modes is as the 0-8 strings for a D0-brane probing a D8-brane at the point $a=a^{0}$ of the dual torus $\widehat{T}^{1}$ (or equivalently infinite array of D8-branes at $a=a^{0}+\hat{n}$ in the covering space). Alternatively, these can also be interpreted as 0-9 strings for a D0-brane probing Type IIA D9-branes where $\mathcal{D}(a)=-i\left(a-\left(a^{0}+\hat{n}\right)\right)$ are interpreted as the tachyon fields of D9-branes as appeared in [17] which represent the infinite array of D8-branes. One can also start with (physical) Type IIB string theory with $\mathrm{D} p$-brane ( $p$ is odd say $9-2 m$ ) wrapped on $T^{1}$ with Wilson line $i a^{0} \mathrm{~d} x$. The $\mathrm{D} p$-brane can be represented as a D9-anti-D9 bound state with tachyon field $T=-i \mathbf{x} \cdot \boldsymbol{\Gamma}$ where $\mathbf{x}=\left(x^{1}, \ldots, x^{2 m}\right)$ are the coordinates transverse to the $\mathrm{D} p$-brane and $\boldsymbol{\Gamma}=\left(\Gamma^{1}, \ldots, \Gamma^{2 m}\right)$ are the Gamma matrices mapping positive chirality spinors to negative chirality spinors in $2 m$-dimensions. Then, 1-9 and $1-\overline{9}$ fermions reduce to fermions with mass matrix

$$
\mathcal{D}(\mathbf{x}, a)=\left(\begin{array}{cc}
-i\left(a-\left(a^{0}+\hat{n}\right)\right) & -i \mathbf{x} \cdot \mathbf{\Gamma}^{\dagger} \\
-i \mathbf{x} \cdot \mathbf{\Gamma} & i\left(a-\left(a^{0}+\hat{n}\right)\right)
\end{array}\right)
$$

This can be expressed as $\mathcal{D}(\vec{x})=-i\left(\vec{x}-\vec{x}_{\hat{n}}\right) \cdot \vec{\Gamma}$ where $\vec{\Gamma}$ are the Gamma matrices for the $(2 m+1)$-dimensions of $\vec{x}=(\mathbf{x}, a)$ and $\vec{x}_{\hat{n}}=\left(\mathbf{0}, a^{0}+\hat{n}\right)$. This 
can be interpreted as the tachyon fields of Type IIA D9-branes representing infinite array of $\mathrm{D}(8-2 m)$-branes located at $\mathbf{x}=\mathbf{0}, a=a^{0}+\hat{n}$. Thus we have recovered the fact that a $\mathrm{D} p$-brane wrapped on a circle is $\mathrm{T}$-dual to a $\mathrm{D}(p-1)$-brane in the dual circle where the Wilson line on the $\mathrm{D} p$-brane corresponds to the position of the $\mathrm{D}(p-1)$-brane $[1,2]$.

As in $T^{4}$ case, the interpretation of the operator $\mathcal{D}$ as the tachyon field of ninebranes in the T-dualized system would lead to an index-theoretic map of K-theory groups that realizes transformation of D-brane charges under T-duality. Note that the operator $\mathcal{D}$ (as well as the Dirac operator $D$ on odd-dimensional space) is skew-adjoint and therefore its family index always vanishes as an element of the $\mathrm{K}$-group of the parameter space. However, Atiyah and Singer in [8] ${ }^{1}$ studied index theory of such families and found that the index naturally takes values in $\mathrm{K}^{-1}$-group of the parameter space. This is actually what we wanted because T-duality maps Type IIB on $T^{1} \times M$ to Type IIA on $\widehat{T}^{1} \times M$ whose D-brane charges are classified by $\mathrm{K}^{-1}\left(\widehat{T}^{1} \times\right.$ $M)$ where $\widehat{T}^{1} \times M$ appears in the present set-up as the parameter space. The construction in [8] is as follows. If $\mathcal{D}(y)$ is the family of skew-adjoint operators parametrized by $y \in Y$, we define a family over $\left[-\frac{\pi}{2}, \frac{\pi}{2}\right] \times Y$ by

$$
\widetilde{\mathcal{D}}(t, y)=-\sin t+\mathcal{D}(y) \cos t
$$

This is no longer skew-adjoint and therefore can have distinct kernel and cokernel. Since $\widetilde{\mathcal{D}}\left(-\frac{\pi}{2}, y\right)=-\widetilde{\mathcal{D}}\left(\frac{\pi}{2}, x\right) \equiv 1$, kernel and cokernel are isomorphic at $t=\mp \frac{\pi}{2}$ and therefore the index of $\widetilde{\mathcal{D}}$ belongs to $\mathrm{K}\left(\left[-\frac{\pi}{2}, \frac{\pi}{2}\right] \times Y, \partial\left[-\frac{\pi}{2}, \frac{\pi}{2}\right] \times\right.$ $Y)=K^{-1}(Y)$.

The element of $\mathrm{K}^{-1}\left(\widehat{T}^{1} \times M\right)$ obtained in this way in our set-up can be identified as the D-brane charge of the T-dualized system. We show this in the example of $\mathrm{D}(9-2 m)$-brane considered above where we put $a^{0}=0$ for simplicity and we compactify the transverse space $\mathbf{R}^{2 m}$ to $S^{2 m}$, requiring triviality at infinity $\infty \in S^{2 m}$ (we thank the authors of [21] for pointing out omission of this requirement in the previous version of the paper). The operator $\widetilde{\mathcal{D}}(t, \vec{x})=-\sin t+\mathcal{D}(\vec{x}) \cos t$ has zero for constant modes at $(t, \vec{x})=(0, \overrightarrow{0})$, and there are no zero modes everywhere else. In a neightborhood of this point, $\widetilde{\mathcal{D}}(t, \vec{x})$ on the constant modes behaves as

$$
\widetilde{\mathcal{D}}(t, \vec{x})=-\sin t-i \vec{x} \cdot \vec{\Gamma} \cos t \approx-(t+i \vec{x} \cdot \vec{\Gamma}) .
$$

\footnotetext{
${ }^{1}$ We thank I.M. Singer for explanation of essential points which are relevant in the present discussion.
} 
This has winding number one on the $(2 m+1)$-sphere surrounding $(t, \vec{x})=$ $(0, \overrightarrow{0})$, and therefore the index of $\widetilde{\mathcal{D}}$ has $\mathrm{ch}_{0}=\cdots=\mathrm{ch}_{2 m}=0, \mathrm{ch}_{2 m+2}=1$ as an element of $\mathrm{K}\left(S^{1} \times \frac{\widehat{T}^{1} \times S^{2 m}}{\widehat{T}^{1} \times \infty}\right)$. This is the generator of $\mathrm{K}^{-1}\left(\widehat{T}^{1} \times S^{2 m}, \widehat{T}^{1} \times\right.$ $\infty) \cong \mathbf{Z}$ which represents the charge of the $\mathrm{D}(8-2 m)$-brane of the T-dualized system. $^{2}$

In the framework where the space-time is compactified by attaching infinity, ${ }^{3}$ the change of the off-diagonal part of (5.6) is a small perturbation and does not affect the index. Therefore, the index depends only on the $\mathrm{K}$ theory class of $\left(E^{+} \otimes \mathcal{P}, E^{-} \otimes \mathcal{P}\right)$ where $E^{-} \otimes \mathcal{P}$ gives a negative contribution because the Dirac operator $D_{-}$enters in $\mathcal{D}$ with minus sign. In particular, it can be denoted as ind $\left(D,\left(E^{+} \otimes \mathcal{P}, E^{-} \otimes \mathcal{P}\right)\right)$. Thus, the map that realizes the transformation of D-brane charges under T-duality can be identified as

$$
\mathrm{K}\left(T^{1} \times M\right) \stackrel{\otimes \mathcal{P}}{\longrightarrow} \mathrm{K}\left(T^{1} \times \widehat{T}^{1} \times M\right) \stackrel{\text { ind } D}{\longrightarrow} \mathrm{K}^{-1}\left(\widehat{T}^{1} \times M\right) .
$$

In [8] it was also shown that the index of a family of skew-adjoint operators on a real Hilbert space takes values in $\mathrm{KO}^{-1}$ of the parameter space via the same construction. A natural generalization of the construction to the case with involution on the parameter space $X$ exists, and the index takes values in $\mathrm{KR}^{-1}(X)$. Thus, we also have

$$
\mathrm{KR}\left(T^{1} \times M\right) \stackrel{\otimes \mathcal{P}}{\longrightarrow} \mathrm{KR}\left(T^{1} \times \widehat{T}^{1} \times M\right) \stackrel{\text { ind } D}{\longrightarrow} \mathrm{KR}^{-1}\left(\widehat{T}^{1} \times M\right),
$$

where the involution acts trivially on $T^{1}$ but as the inversion on $\widehat{T}^{1}$.

What if we started from an element of $\mathrm{K}^{-1}\left(T^{1} \times M\right)$ ? Such an element can be considered as an element of $\mathrm{K}\left(S^{1} \times T^{1} \times M\right)$ that vanishes on $z_{0} \times$ $T^{1} \times M$. Tensoring with $\mathcal{P}$ and taking the index do not affect the triviality at $z_{0} \in S^{1}$. Thus, its image under the map (5.10), which a priori belongs to

\footnotetext{
${ }^{2}$ In [17], the element of $\mathrm{K}^{-1}(X)$ corresponding to the (anti-hermiaitin) tachyon field $\mathcal{T}: E \rightarrow E$ of IIA ninebrane is identified as $\left(E, \mathrm{e}^{\mathcal{T}}\right)$ in the definition of $\mathrm{K}^{-1}(X)$ in [45]. It is $\left(E_{\mathrm{e}} \tau, E_{\mathrm{id}}\right)$ in the standard definition of $\mathrm{K}^{-1}(X)$ as the subgroup of $\mathrm{K}\left(S^{1} \times X\right)$, where $E_{\varphi}$ for an auatomorphism $\varphi: E \rightarrow E$ is a bundle over $S^{1} \times X$ defined as [0,1] $\times E$ modulo the identification $(0, v) \equiv(1, \varphi v)$. In our set-up, after a suitable regularization of $\mathcal{T}=\mathcal{D}, \mathrm{e}^{\mathcal{T}}$ has winding number one on a disc $D^{2 m+1}$ containing $\vec{x}=\overrightarrow{0}$ (i.e. it is constant on $\partial D^{2 m+1}$ and the induced map on $S^{2 m+1}=D^{2 m+1} / \partial D^{2 m+1}$ has winding number one). Therefore $E_{\mathrm{e}} \mathcal{T}$ and $E_{\text {id }}$ differ in $\mathrm{ch}_{2 m+2}$ by one, determining the same element as the index of $\widetilde{\mathcal{D}}$.

${ }^{3}$ Triviality at infinity is imposed as usual but, as in [15], is not explicitly indicated in the general formula since what "infinity" means depends on (and is clear in) the particular situation one considers.
} 
$\mathrm{K}^{-1}\left(S^{1} \times \widehat{T}^{1} \times M\right)$, actually belongs to the subgroup $\mathrm{K}^{-2}\left(\widehat{T}^{1} \times M\right)$. Such a consideration leads to the following generalizations of (5.10) and (5.11)

$$
\begin{aligned}
& \mathrm{K}^{-i}\left(T^{1} \times M\right) \stackrel{\otimes \mathcal{P}}{\longrightarrow} \mathrm{K}^{-i}\left(T^{1} \times \widehat{T}^{1} \times M\right) \stackrel{\text { ind } D}{\longrightarrow} \mathrm{K}^{-i-1}\left(\widehat{T}^{1} \times M\right),(5.12) \\
& \mathrm{KR}^{-i}\left(T^{1} \times M\right) \stackrel{\otimes \mathcal{P}}{\longrightarrow} \mathrm{KR}^{-i}\left(T^{1} \times \widehat{T}^{1} \times M\right) \stackrel{\text { ind } D}{\longrightarrow} \mathrm{KR}^{-i-1}\left(\widehat{T}^{1} \times M\right) .
\end{aligned}
$$

By composition, we obtain maps

$$
\begin{aligned}
& \mathrm{K}^{-i}\left(T^{n} \times M\right) \longrightarrow \mathrm{K}^{-i-n}\left(\widehat{T}^{n} \times M\right), \\
& \mathrm{KR}^{-i}\left(T^{n} \times M\right) \longrightarrow \mathrm{KR}^{-i-n}\left(\widehat{T}^{n} \times M\right),
\end{aligned}
$$

representing T-duality on $n$-torus where now $M$ is a $(10-n)$-dimensional manifold. In the latter case, the involution acts trivially on $T^{n}$ and as the inversion on $\widehat{T}^{n}$ while $M$ is assumed to have a suitable involution so that $i$ can be chosen appropriately.

As a consistency check, let us consider Type I string on $T^{n} \times M$ which T-dualizes to Type II orientifold on $\widehat{T}^{n} / \mathbf{Z}_{2} \times M$ of $S O$-type. Type I Dbrane charge takes values in $\mathrm{KO}\left(T^{n} \times M\right)=\mathrm{KR}\left(T^{n} \times M\right)$. Therefore, by (5.15), we see that D-brane charges of Type II orientifold on $\widehat{T}^{n} / \mathbf{Z}_{2} \times M$ must be classified by $\mathrm{KR}^{-n}\left(\widehat{T}^{n} \times M\right)$, thus reproducing our proposal (for $S O$-type orientifold). Also, when involution acts on $M$ with only $\mathbf{R}^{s} / \mathbf{Z}_{2^{-}}$ type fixed points, according to our proposal, D-brane charges of orientifold on $T^{n} \times M / \mathbf{Z}_{2}$ are classified by $\mathrm{KR}^{-i}\left(T^{n} \times M\right)$ with $i=s$ or $s+4$ for $S O$ or $S p$-type respectively. Then (5.15) shows that D-branes in orientifold on $\left(\widehat{T}^{n} \times M\right) / \mathbf{Z}_{2}$ is classified by $\mathrm{KR}^{-j}\left(\widehat{T}^{n} \times M\right)$ with $j=s+n$ or $s+n+4$, which is consistent with our proposal since there are only $\mathbf{R}^{n+s} / \mathbf{Z}_{2}$ fixed points.

Since T-duality squares to the identity, the maps we have obtained must be isomorphisms. Here we do not attempt to compute these isomorphisms. Instead, we show that the groups are indeed isomorphic.

\section{K-theory Groups of $T^{n} \times M$}

A subspace $B$ of a topological space $A$ is said to be a retract of $A$ if there is a continuous map $f: A \rightarrow B$ that restricts on $B$ to the identity. In such a case, there is a relation $\mathrm{K}(A)=\mathrm{K}(A, B) \oplus \mathrm{K}(B)$ which holds also for $\mathrm{K}^{-i}$ 
and $\mathrm{KR}^{-p,-q}$. Now let us apply this to $z_{0} \times M \subset T^{1} \times M$ which is obviously a retract. Noting that $K^{-i}\left(T^{1} \times M, z_{0} \times M\right)=K^{-i-1}(M)$, we have

$$
\mathrm{K}^{-i}\left(T^{1} \times M\right)=\mathrm{K}^{-i-1}(M) \oplus \mathrm{K}^{-i}(M) .
$$

To consider the case with invoultion, as before we let $\mathbf{Z}_{2}$ act on $T^{n}$ trivially and on $\widehat{T}^{n}$ as the inversion. Then, $z_{0} \times M \subset T^{1} \times M$ and $\hat{z}_{0} \times M \subset \widehat{T}^{1} \times M$ are both retracts where $\hat{z}_{0}$ is a $\mathbf{Z}_{2}$ fixed point. Noting that $\mathrm{KR}^{-p,-q}\left(T^{1} \times M, z_{0} \times\right.$ $M)=\mathrm{KR}^{-p-1,-q}(M)$ and $\mathrm{KR}^{-p,-q}\left(\widehat{T}^{1} \times M, \hat{z}_{0} \times M\right)=\mathrm{KR}^{-p,-q-1}(M)$, we have

$$
\begin{aligned}
& \mathrm{KR}^{-p,-q}\left(T^{1} \times M\right)=\mathrm{KR}^{-p-1,-q}(M) \oplus \mathrm{KR}^{-p,-q}(M), \\
& \mathrm{KR}^{-p,-q}\left(\widehat{T}^{1} \times M\right)=\mathrm{KR}^{-p,-q-1}(M) \oplus \mathrm{KR}^{-p,-q}(M) .
\end{aligned}
$$

Using these relations repeatedly, we obtain the binomial identities

$$
\begin{aligned}
& \mathrm{K}^{-i}\left(T^{n} \times M\right)=\bigoplus_{k=0}^{n} \mathrm{~K}^{-i-k}(M)^{\oplus\left(\begin{array}{c}
n \\
k
\end{array}\right)}, \\
& \mathrm{KR}^{-p,-q}\left(T^{n} \times M\right)=\bigoplus_{k=0}^{n} \mathrm{KR}^{-p-k,-q}(M)^{\oplus\left(\begin{array}{l}
n \\
k
\end{array}\right)}, \\
& \mathrm{KR}^{-p,-q}\left(\widehat{T}^{n} \times M\right)=\bigoplus_{k=0}^{n} \mathrm{KR}^{-p,-q-k}(M)^{\oplus\left(\begin{array}{l}
n \\
k
\end{array}\right)},
\end{aligned}
$$

where $M$ is any space (with an involution in the latter two). Using Bott periodicity $\mathrm{K}^{-j-2}=\mathrm{K}^{-j}, \mathrm{KR}^{-p-1,-q-1}=\mathrm{KR}^{-p,-q}$, we see from these that

$$
\begin{aligned}
& \mathrm{K}^{-i}\left(T^{n} \times M\right) \cong \mathrm{K}^{-i-n}\left(\widehat{T}^{n} \times M\right), \\
& \mathrm{KR}^{-i}\left(T^{n} \times M\right) \cong \mathrm{KR}^{-i-n}\left(\widehat{T}^{n} \times M\right) .
\end{aligned}
$$

Thus, the groups in the left hand side and the right hand side of the T-duality maps (5.14) and (5.15) are indeed isomorphic.

Applying the binomial identities (5.19)-(5.21) to the case where $M$ is a point, let us compute some K-groups of torus. We first note that $\mathrm{K}(\mathrm{pt})=\mathbf{Z}$ and $\mathrm{K}^{-1}(\mathrm{pt})=0$. Then, we immediately obtain from (5.19)

$$
\mathrm{K}\left(T^{n}\right) \cong \mathbf{Z}^{\oplus 2^{n-1}}, \quad \mathrm{~K}^{-1}\left(T^{n}\right) \cong \mathbf{Z}^{\oplus 2^{n-1}} .
$$

These are mapped isomorphically by the Chern character maps ch $: \mathrm{K}(X) \rightarrow$ $H^{\text {even }}(X, \mathbf{Q}), \mathrm{K}^{-1}(X) \rightarrow H^{\text {odd }}(X, \mathbf{Q})$ to the even and odd dimensional integral cohomology groups of the torus respectively (or more precisely their 
images in the rational cohomology). Note that $2^{n-1}$ is the dimension of the (positive or negative) spinor representation of $S O(2 n)$. In fact, it is known that the T-duality group $O(n, n ; \mathbf{Z})$ acts on the RR potentials in the spinor representation, where IIA potentials belong to, say, positive spinor representation while IIB potentials belong to negative spinor representation which are interchanged under odd number of T-duality on circles [46]. This fact may be explained by showing that our T-duality maps for D-brane charges (together with the mapping class group of $T^{n}$ and some operation corresponding to B-field shift) generate spinor representation of $O(n, n ; \mathbf{Z})$.

To compute $\mathrm{KR}^{-i}\left(T^{n}\right)$, we need to know $\mathrm{KR}^{-i}(\mathrm{pt})=\mathrm{KO}^{-i}(\mathrm{pt})$. Nonzero groups of them are $\mathrm{KO}(\mathrm{pt})=\mathbf{Z}, \mathrm{KO}^{-1}(\mathrm{pt})=\mathbf{Z}_{2}, \mathrm{KO}^{-2}(\mathrm{pt})=\mathbf{Z}_{2}$ and $\mathrm{KO}^{-4}(\mathrm{pt})=\mathbf{Z}$. Inserting these into the identities (5.20) and (5.21) we obtain for example

$$
\begin{aligned}
& \mathrm{KO}\left(T^{4}\right)=\mathbf{Z} \oplus \mathbf{Z}_{2}^{\oplus 4} \oplus \mathbf{Z}_{2}^{\oplus 6} \oplus \mathbf{Z}=\mathrm{KpR}\left(\widehat{T}^{4}\right), \\
& \mathrm{KSp}\left(T^{4}\right)=\mathbf{Z} \oplus \mathbf{Z}=\mathrm{KR}\left(\widehat{T}^{4}\right) .
\end{aligned}
$$

It is intereting to investigate what the full T-duality group is and in which representation the $\mathrm{D}$-brane charges (and RR fields) belong to. 


\section{Appendix}

\section{A The Inversion Theorem}

In this appendix we show that square Nahm transform is the identity operation for orthogonal/symplectic (orienti)bundles over $T^{4}\left(T^{4} / \mathbf{Z}_{2}\right)$ or for unitary orbibundles over orbifold $T^{4} / \mathbf{Z}_{2}$. Namely, we prove that there is an isomorphism

$$
(E, A, \Sigma) \stackrel{\cong}{\longrightarrow}(E, A, \Sigma)^{\sim}
$$

where $\Sigma$ is either one of

(i) the orthogonal structure defined by anti-linear maps $I_{x}: E_{x} \rightarrow E_{x}$

(ii) the symplectic structure defined by anti-linear maps $J_{x}: E_{x} \rightarrow E_{x}$

(iii) the $\mathbf{Z}_{2}$-orbifold action defined by linear maps $\varphi_{x}: E_{x} \rightarrow E_{-x}$

on the unitary bundle $E$ over $T^{4}$, and "^" and " " " are the Nahm transforms which we have constructed in sections 3 ( ${ }^{\circ}$ is equal to ${ }^{\wedge}$ for the case (iii)). It is already known that there is an isomorphism $(E, A) \cong(E, A)^{\sim}[10,12]$ (see also[13]) if we ignore the respective structure $\Sigma$. Therefore, what we need to do is to show that this isomorphism sends the structure $\Sigma$ to that of the square Nahm transform. We present the proof for the case (i) in some detail, and only indicate the essential points for the cases (ii) and (iii).

We first recall the definition of the isomorphism $u: E \rightarrow \hat{E}$ [12]. Let us denote by $G_{a}$ the inverse of the Laplace operator $D^{(a) \dagger} D^{(a)}$ associated with the covariant derivative $D_{\mu}^{(a)}=\partial_{\mu}+A_{\mu}-i a_{\mu}$. Then for $v \in E_{x}, u(v) \in(\hat{E})_{x}$ is given as the section of the bundle $\hat{E} \otimes \hat{S}^{+}$defined by

$$
u(v): a \longmapsto \sqrt{-1}\left[\psi_{i}^{(a)}\right] \otimes C\left(\left(G_{a} \psi_{i}^{(a)}\right)(x), v\right)
$$

where $\left\{\psi_{i}^{(a)}\right\}$ is an orthonormal basis of the kernel of the Dirac operator $\not D^{(a)}$ (representing an orthonormal basis $\left\{\left[\psi_{i}^{(a)}\right]\right\}$ of $\hat{E}_{a}$ ), $C$ is the charge conjugation matrix and $($,$) is the hermitain metric of E$ which is anti-linear in the left-entry and linear in the right-entry. (The phase $\sqrt{-1}$ in front is simply for later convenience.) To be precise, (A.2) defines a section of $\hat{E} \otimes \mathcal{L}_{x} \otimes \hat{S}^{+}$where $\mathcal{L}_{x}$ is a flat bundle defined as the quotient of $\left(\mathbf{R}^{4}\right)^{*} \times \mathbf{C}$ with the trivial connection by the $2 \pi \Lambda^{*}$ action $(a, c) \mapsto\left(a+\hat{n}, \mathrm{e}^{-i \hat{n} x} c\right)$. (This is because $\psi^{(a)}(x) \in \operatorname{Ker} \not D^{(a)}$ and $\psi^{(a+\hat{n})}(x)=\mathrm{e}^{i \hat{n} x} \psi^{(a)}(x) \in \operatorname{Ker} \not D^{(a+\hat{n})}$ are 
identified as an element of $\hat{E}_{a}$.) However, $\mathcal{L}_{x}$ is topologically trivial and is isomorphic as a flat bundle to the trivial bundle $\widehat{T}^{4} \times \mathbf{C}$ with the connection $D=d-i x^{\mu} \mathrm{d} a_{\mu}$. By this identification, we consider $u(v)$ as a section of $\hat{E} \otimes \hat{S}^{+}$. One can show, as in [12], that $u(v)$ is annihilated by the Dirac operator associated with the covariant derivative $\partial / \partial a_{\mu}+\hat{A}^{\mu}-i x^{\mu}$, thus showing that $u(v)$ belongs to $(\hat{E})_{x}$. Moreover, it was shown in [12] that $u$ sends the hermitian metric of $E$ to that of $\hat{E}$ and the connection $A$ to $\tilde{\hat{A}}$.

Now let us show that $u$ sends the orthogonal structure $I$ of $(E, A)$ to that $\check{I}$ of $(E, A)^{\varkappa}$. Namely we show that

$$
\check{I}_{x} u(v)=u\left(I_{x} v\right)
$$

for each $v \in E_{x}$. By definition, we have $\left.\check{I} u(v)\right|_{a}=J_{-a}^{c}\left(\left.u(v)\right|_{-a}\right)$, and therefore

$$
\begin{aligned}
\left.\check{I} u(v)\right|_{a} & =-\sqrt{-1} J_{-a}\left(\left[\psi_{i}^{(-a)}\right]\right) \otimes \gamma^{1234}\left[C\left(\left(G_{-a} \psi_{i}^{(-a)}\right)(x), v\right)\right]^{c} \\
& =\sqrt{-1}\left[I^{c} \psi_{i}^{(-a)}\right] \otimes C \bar{C}\left(v,\left(G_{-a} \psi_{i}^{(-a)}\right)(x)\right) \\
& =\sqrt{-1}\left[I^{c} \psi_{i}^{(-a)}\right] \otimes C \bar{C}\left(I_{x}\left(G_{-a} \psi_{i}^{(-a)}\right)(x), I_{x} v\right) \\
& =\sqrt{-1}\left[I^{c} \psi_{i}^{(-a)}\right] \otimes C\left(C\left(I G_{-a} \psi_{i}^{(-a)}\right)(x), I_{x} v\right),
\end{aligned}
$$

where $I$ is considered as an operator acting on the section $s(x)$ of $E$ as $(I s)(x):=I_{x} s(x)$. Note that $I G_{-a}=G_{a} I$ which follows from $I D^{(a)}=$ $D^{(-a)} I$. Then, we see that

$$
C\left(I G_{-a} \psi^{(-a)}\right)(x)=C G_{a} I \psi^{(-a)}(x)=G_{a} I^{c} \psi^{(-a)}(x) .
$$

Inserting this to (A.4), we have shown (A.3):

$$
\begin{aligned}
\left.\check{I} u(v)\right|_{a} & =\sqrt{-1}\left[I^{c} \psi_{i}^{(-a)}\right] \otimes C\left(\left(G_{a} I^{c} \psi_{i}^{(-a)}\right)(x), I_{x} v\right) \\
& =\left.u\left(I_{x} v\right)\right|_{a}
\end{aligned}
$$

thus proving (A.1).

The proof for the cases of (ii) and (iii) are similar. The essential points in these cases are $J G_{-a}=G_{a} J$ for (ii) and $\varphi G_{-a}=G_{a} \varphi$ for (iii), where $J$ and $\varphi$ are considered as an operator acting on the section $s$ of $E$ as $(J s)(x)=J_{x} s(x)$ and $(\varphi s)(x)=\varphi_{-x} s(-x)$ respectively. 


\section{Acknowledgements}

I would like to thank B. Acharya, J. Blum, J. de Boer, S. Kachru, A. Karch, J. Maldacena, N. Obers, H. Ooguri, A. Strominger, C. Vafa and P. Yi for useful discussions and M. Hopkins, G. Segal and I.M. Singer for help and instructions in K-theory and index theory. I wish to thank Aspen Center for Physics, Physics and Mathematics Departments of Harvard University, and Korea Institute for Advanced Study, where various stages of this work were carried out, for their kind hospitality. I express my thanks and apologies to many people who kindly pointed out omission and incorrect citation in the previous version of the paper.

This research is supported in part by NSF grant PHY-95-14797 and DOE grant DE-AC03-76SF00098.

\section{References}

[1] J. Dai, R.G. Leigh, and J. Polchinski, "New Connections Between String Theories", Mod.Phys.Lett. A4 (1989) 2073-2083.

[2] P. Hořava, "Strings on Worldsheet Orbifolds", Nucl.Phys. B327 (1989) 461484; "Background Duality of Open String Models", Phys.Lett. 231B (1989) 251-257.

[3] J. Polchinski, "Dirichlet-Branes and Ramond-Ramond Charges", hepth/9510017, Phys. Rev.Lett. 75 (1995) 4724-4727.

[4] E. Bergshoeff, C.M. Hull and T. Ortin, "Duality in the Type-II Superstring Effective Action", hep-th/9504081, Nucl.Phys. B451 (1995) 547.

[5] E. Eyras, B. Janssen and Y. Lozano, "5-branes, KK-monopoles and T-duality", hep-th/9806169, Nucl.Phys. B531 (1998) 275-301.

[6] M.F. Atiyah and I.M. Singer, "The Index of Elliptic Operators IV" Ann.Math. 93 (1971) 119-138.

[7] M.F. Atiyah and I.M. Singer, "The Index of Elliptic Operators V" Ann.Math. 93 (1971) 139-149.

[8] M.F. Atiyah and I.M. Singer, "Index Theory for Skew-adjoint Fredholm Operators", Publ.Math. I.H.E.S. 37 (1969) 305-326.

[9] M.R. Douglas and G. Moore, "D-branes, Quivers, and ALE Instantons", hepth/9603167. 
[10] S. Mukai, "Duality between $\mathbf{D}(X)$ and $\mathbf{D}(\widehat{X})$ with its application to Picard Sheaves", Nagoya Math.J. 81 (1981) 153-175.

[11] H. Shenk, "On a Generalized Fourier Transform of Instantons over Flat Tori", Commun.Math.Phys. 116 (1988) 177.

[12] P.J. Braam and P. van Baal, "Nahm's Transform for Instanton", Commun.Math.Phys. 122 (1989) 267.

[13] S.K. Donaldson and P.B. Kronheimer, The Geometry of Four-Manifolds, Clarendon Press, Oxford, 1990.

[14] A. Sen, "Stable Non-BPS States in String Theory", hep-th/9803194, JHEP 9806 (1998) 007; "Stable Non-BPS Bound States of BPS D-branes", hepth/9805019, JHEP 9808 (1998) 010; "Tachyon Condensation on the Brane Antibrane System", hep-th/9805170, JHEP 9808 (1998) 012; "SO(32) Spinors of Type I and Other Solitons on Brane-Antibrane Pair", hep-th/9808141, JHEP 9809 (1998) 023; "Type I D-particle and its Interactions", hep-th/9809111, JHEP 9810 (1998) 021;

O. Bergman, M.R. Gaberdiel, "Stable non-BPS D-particles", hep-th/9806155, Phys.Lett. 441B (1998) 133-140.

[15] E. Witten, " $D$-Branes And K-Theory", hep-th/9810188.

[16] R. Minasian and G. Moore, "K-theory and Ramond-Ramond charge", hepth/9710230, JHEP 9711 (1997) 002.

[17] P. Hořava, "Type IIA D-Branes, K-Theory, and Matrix Theory", hepth/9812135.

[18] H. García-Compeán, "D-branes in Orbifold Singularities and Equivariant KTheory", hep-th/9812226.

[19] S. Gukov, "K-Theory, Reality, and Orientifolds", hep-th/9901042.

[20] E.R. Sharpe, "D-Branes, Derived Categories, and Grothendieck Groups", hepth/9902116.

[21] O. Bergman, E. Gimon and P. Hořava, "Brane Transfer Operations and Tduality of Non-BPS States", hep-th/9902160.

[22] M. R. Douglas, "Branes within Branes", hep-th/9512077.

[23] J.A. Harvey and G. Moore, "On the algebras of BPS states", hep-th/9609017, Commun.Math.Phys. 197 (1998) 489-519.

[24] J. Polchinski and E. Witten, "Evidence for Heterotic - Type I String Duality", hep-th/9510169, Nucl.Phys. B460 (1996) 525.

[25] E. Witten, "Small Instantons in String Theory", hep-th/9511030, Nucl.Phys. B460 (1996) 541-559.

[26] M. R. Douglas, "Gauge Fields and D-branes", hep-th/9604198. 
[27] E. Witten, "Sigma Models And The ADHM Construction Of Instantons", hep-th/9410052, J.Geom.Phys. 15 (1995) 215.

[28] D-E. Diaconescu, "D-branes, Monopoles and Nahm Equations", Nucl.Phys. B503 (1997) 220-238, hep-th/9608163.

[29] A. Kapustin and S. Sethi, "The Higgs Branch of Impurity Theories", hepth/9804027, Adv.Theor.Math.Phys. 2 (1998) 571-591.

[30] D. Tsimpis, "Nahm Equations and Boundary Conditions", hep-th/9804081, Phys.Lett. 433B (1998) 287-290.

[31] A. Strominger, S-T. Yau and E. Zaslow, "Mirror Symmetry is T-Duality", hep-th/9606040, Nucl.Phys. B479 (1996) 243-259.

[32] R. Hartshorne, Residues and Duality, Lect. Notes in Math. 20, SpringerVerlag, Berlin-Heidelberg-New York, 1966.

[33] W. Taylor, "D-brane field theory on compact spaces", hep-th/9611042, Phys.Lett. B394 (1997) 283-287.

[34] T. Banks and L. Motl, "Heterotic Strings from Matrices", hep-th/9703218, J. High Energy Phys. 12 (1997) 004.

[35] P. Hořava, "Matrix Theory and Heterotic Strings on Tori", hep-th/9705055, Nucl.Phys. B505 (1997) 84-108.

[36] N. Kim and S.-J. Rey, "M(atrix) Theory on T5/Z2 Orbifold and Five-Branes", hep-th/9705132, Nucl.Phys. B534 (1998) 155-182.

[37] S. Ramgoolam and D. Waldram, "Zero-Branes on a Compact Orbifold", hepth/9805191, J.High Energy Phys. 07 (1998) 009.

[38] B.R. Greene, C.I. Lazaroiu and P. Yi, "D-particles on $T^{4} / Z_{n}$ orbifolds and their resolutions", hep-th/9807040.

[39] K. Hori and Y: Oz, "F-Theory, T-Duality on K3 Surfaces and N=2 Supersymmetric Gauge Theories in Four Dimensions", hep-th/9702173, Nucl.Phys. B501 (1997) 97-108.

[40] M. Srednicki, "IIB or not IIB", hep-th/9807138, JHEP 9808 (1998) 005.

[41] P. Yi, "Membranes from Five-Branes and Fundamental Strings from $\mathrm{D} p$ Branes", hep-th/9901159.

[42] A. Sen, "BPS D-branes on Non-supersymmetric Cycles", hep-th/9812031.

[43] M.F. Atiyah, K-theory, Benjamin, New York, 1967.

[44] M.F. Atiyah, "K-theory and reality", Quart.J.Math. 17 (1966) 367-386.

[45] M. Karoubi, K-theory, An Introduction, Springer-Verlag, Berlin-HeidelbergNew York, 1978.

[46] N.A. Obers and B. Pioline, "U-duality and M-Theory", hep-th/9809039, to appear in Phys. Rep. 
[47] M.F. Atiyah, R. Bott and A. Shapiro, "Clifford Modules", Topology 3 (Suppl. 1) (1964) 3-38. 